\title{
Visualizations of Arterial Traffic Performance Measures: A Picture Book Approach
}
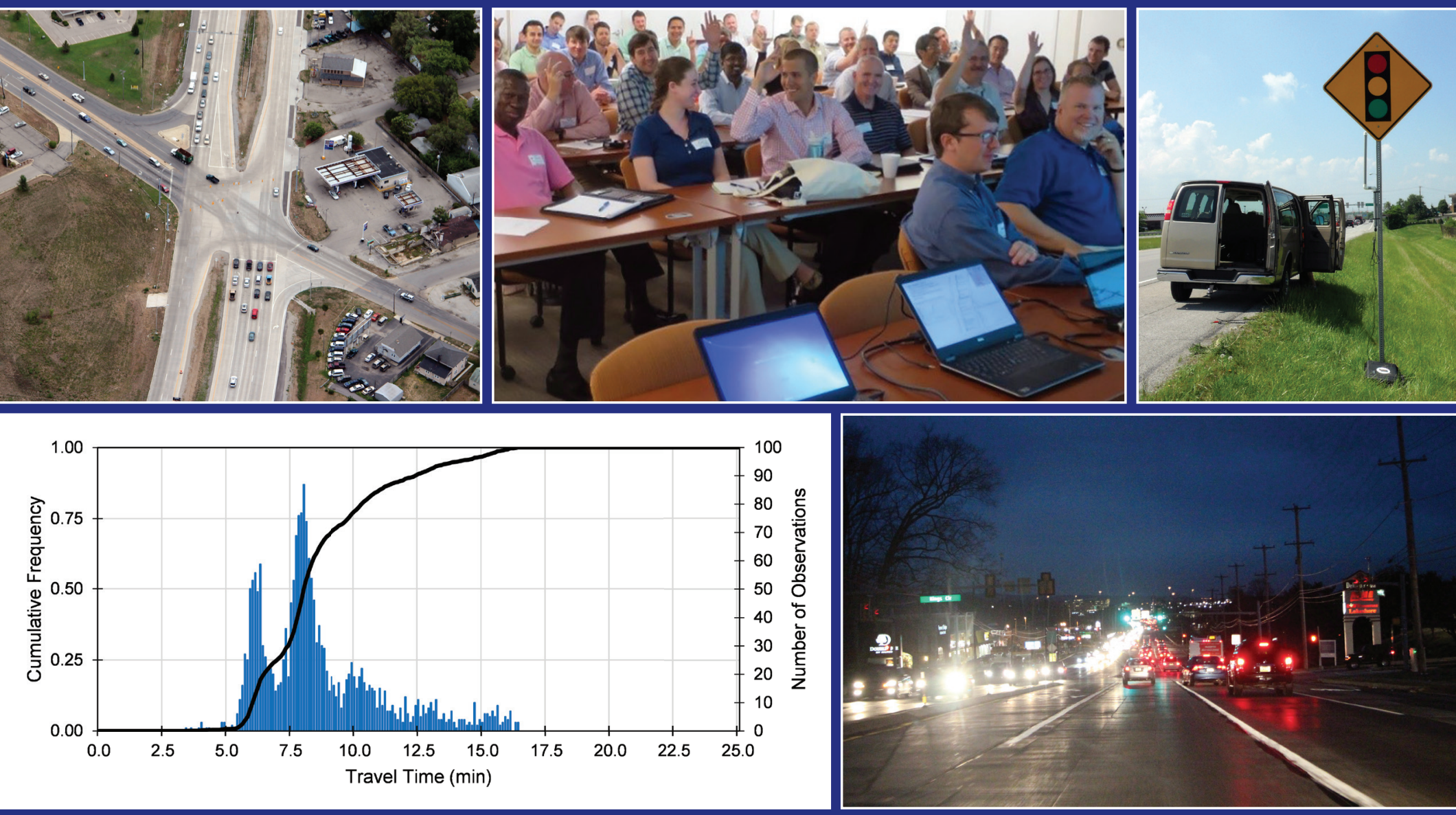

Stanley E. Young, Christopher M. Day, Darcy M. Bullock, Dennis So Ting Fong 


\title{
Visualizations of Arterial Traffic Performance Measures: A Picture Book Approach
}

\author{
Stanley E. Young \\ Traffax, Inc. \\ Christopher M. Day \\ Purdue University \\ Darcy M. Bullock \\ Purdue University \\ Dennis So Ting Fong \\ Traffax, Inc.
}
SBIR Phase 3 Joint Transportation Research Project
Traffax, Inc.
Purdue University

March 4, 2016

\begin{tabular}{|l|l|}
\hline Deliverable Reference: & D1.7 Graphic Performance Measures Report \\
\hline Project Name: & $\begin{array}{l}\text { Sensor Fusion and MOE Development for Off-Line } \\
\text { Traffic Analysis of Real Time Data }\end{array}$ \\
\hline Contractor: & Traffax, Inc. \\
\hline Contract Number: & DTFH61-14-C-00035 \\
\hline Contract Term Start & $9 / 4 / 2014$ \\
\hline Contract Term End & $9 / 4 / 2017$ \\
\hline Key Personnel & Stan Young, Darcy Bullock, Dennis So Ting Fong \\
\hline
\end{tabular}




\section{Recommended Citation}

Young, S. E., C. M. Day, D. M. Bullock, and D. S. T. Fong. Visualizations of Arterial Traffic Performance Measures: A Picture Book Approach. Purdue University, West Lafayette, Indiana, 2017. https://doi. org/10.5703/1288284316562

\section{Acknowledgments}

This work was supported by Traffax/USDOT SBIR DTFH6114C00035. The contents of this paper reflect the views of the authors, who are responsible for the facts and the accuracy of the data presented herein, and do not necessarily reflect the official views or policies of the sponsoring organizations. These contents do not constitute a standard, specification, or regulation. 


\section{Table of Contents}

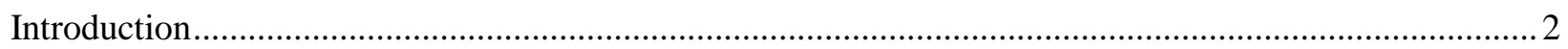

Chapter 1: Visualizations of Travel Time Re-identification Data ................................................... 4

1.1 Construction of Overlay and Cumulative Distribution Graphs.............................................. 4

1.2 Variation of Travel Time on Arterials Using Overlay and CFD Charts ................................ 9

1.3 Signal Controller Attributes Observable in Overlay Charts.................................................. 14

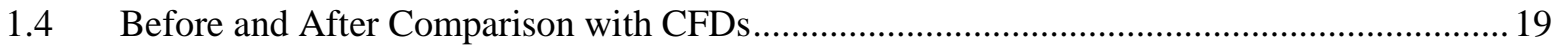

Chapter 2: Quality of Progression with Purdue Coordination Diagrams................................................ 24

2.1 Construction of Purdue Coordination Diagrams from High-Resolution Data ....................... 24

2.2 Signal Control Attributes Observable in PCDs.................................................................... 30

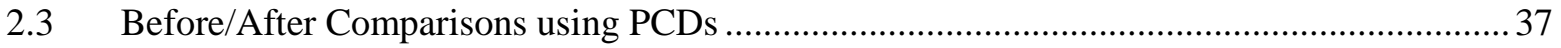

Chapter 3: Capacity Utilization with ROR and GOR Plots ................................................................... 49

3.1 Calculation of ROR and GOR for Determining Split Failure Occurrences ........................... 49

3.2 Intersection and Corridor Analysis of Split Failures.............................................................. 55

3.3 Longitudinal System Analysis of Split Failures................................................................. 60

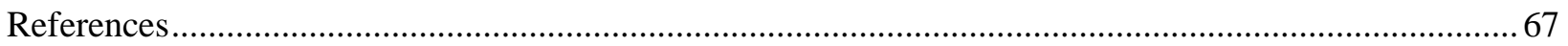

Graphic Performance Measures Report 1 


\section{Introduction}

This report provides a visual reference for the construction and interpretation of key arterial performance measures graphical illustrations. This report is intended as a companion document to the full report Performance Measures of Interrupted-Flow Roadways using Re-identification and Signal Controller Data [1]. Whereas the full report [1] introduced the performance metrics and provided case study examples to explain their use and utility, this companion document concentrates on visualizations of the three key performance metrics, providing a set of reference examples that span the scope of the patterns and characteristic signatures that may be present. The performance measures and their visualizations include:

- Travel time and travel time reliability as revealed through overlay charts and statistical distribution as revealed through cumulative frequency diagrams (CFDs). Overlay charts depict individual vehicle travel times over a 24 hour period accumulated from multiple days. Overlaying multiple days of data reinforces the visible signatures of repeatable traffic patterns induced by signal control. A CFD, also known as cumulative distribution functions in statistical literature, portrays the travel time distribution for specific time intervals. The time interval may be hourly, peak periods, or times periods specific to signal timing plans that are in effect. Combined, overlay charts and CFDs provide visual tools to assess the quality and consistency of traffic movement for various periods throughout the day.

- Quality of platoon progression through a corridor with coordinated signals. The foundation graphic for visualizing progression is the Purdue Coordination Diagram (PCD) . The PCD is constructed based upon arrival times with respect to the green phase collected through highresolution controller data. When PCDs are presented in a series for an entire corridor, they can be used to identify the root causes of poor progression.

- Capacity utilization can be assessed by the red occupancy ratio (ROR) in concert with the green occupancy ratio (GOR) for each signal cycle. If both ratios are over 0.80 for a single phase of an intersection, it indicates a high likelihood that the green phase was unable to fully discharge queued vehicles, resulting in a phase (or split) failure. When ROR is plotted against GOR for multiple signal cycles, the resulting graphic illustrates the frequency of over-saturated conditions resulting in phase failures at the intersection.

These performance measures were introduced, and their meaning and formulation discussed in detail, in [1] using case studies. If the reader is unfamiliar with arterial performance measures obtained through reidentification and high-resolution controller data, they are referred to [1] for foundational information. In contrast to [1], this report provides a visual reference of the breadth of arterial performance phenomenon that has been observed through use of these graphical tools. This report is intended as an educational resource, as well as a reference to interpret a variety of results that may be encountered.

This document is organized into three sections, consistent with the three primary visualizations provided above. For each of the three key visualizations, a brief introduction to the basis of the performance metric is presented as well as the fundamentals of constructing the visualization. After the brief introduction for each metric and its visualization, a series of representative results are presented for various traffic and 
signal operation phenomena that have been observed. These samples were selected to present the breadth of results that may be encountered. Samples include both common and some not-so-common results. Summary information is provided for each segment, intersection or corridor used in the sample performance measures graphics. Emphasis is placed on interpreting (or diagnosing) the broad set of conditions that may be revealed by re-identification data and high-resolution controller data, rather than in-depth analysis or formulation of a remedy. 


\section{Chapter 1: Visualizations of Travel Time Re-identification Data}

\subsection{Construction of Overlay and Cumulative Distribution Graphs}

Vehicle re-identification data measures traffic performance for a segment or a corridor by sampling the travel time of vehicles that traverse the corridor. This is accomplished by identifying a vehicle at two locations using a sensor, and logging the locations and exact time of observation. Re-identification data may come from a variety of technologies. The most common is re-identification data derived from Bluetooth and Wi-Fi consumer electronics. It has become the most common type of re-identification data due to the relatively low cost of equipment and deployment. License plate matching is another form, but typically much more costly, limiting its prevalence. Re-identification techniques can also be used with data from electronic tolling equipment. However, this method requires extra caution as tolling data contains personally identifiable information associated with a customer's account (where as Bluetooth and Wi-Fi data do not.) Connected vehicle data is anticipated to be a significant source of reidentification data in the future. The visualization methods presented below can be used with any source of re-identification data.

Re-identification data collected from Maryland State Route 355, as shown in Figure 1.1.1, is used to illustrate the basic visualizations of travel time overlay and cumulative frequency diagrams (CFDs). The locations of signalized intersections on this segment are indicated by the red markers while the blue markers indicate two data collection devices at the endpoints where permanent Bluetooth traffic recorders are installed. This 2.7 mile corridor is characterized by at least three traffic lanes in each direction, average annual daily traffic slightly above 50,000 vehicles, thirteen signals (not including signalized intersections at the end points), and numerous access points for businesses and side streets. Data from this installation is used as a primer for the development of overlay plots and CFDs.

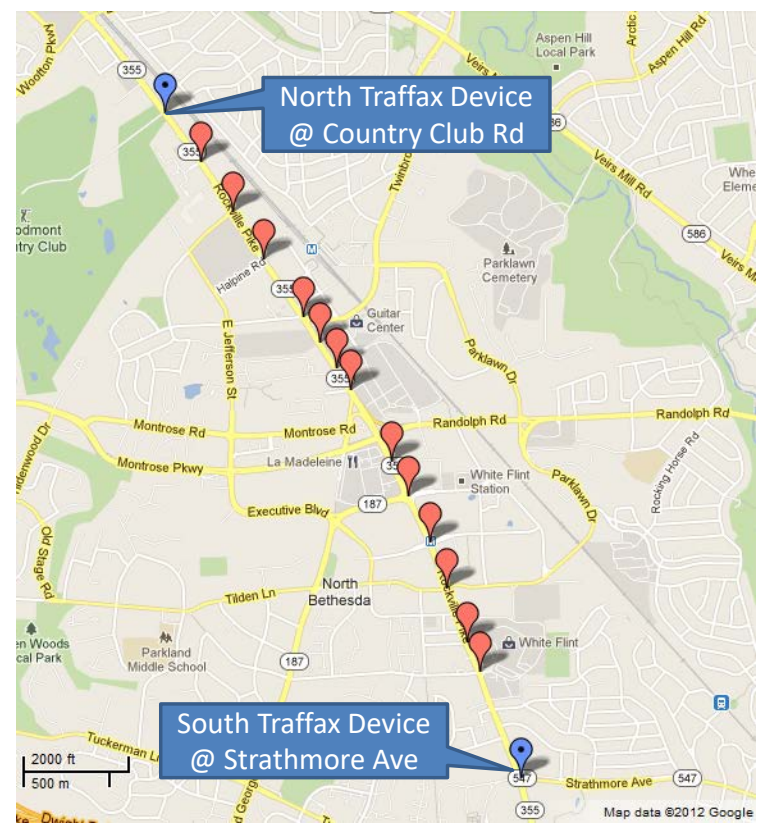

Figure 1.1.1 Illustrative corridor for vehicle re-identification travel times 
The travel time data collected using Bluetooth re-identification sensors is depicted in Figure 1.1.2 for the period from October 2011 to April of 2012, which encompasses approximately 50,000 individual observations of vehicle travel time. This data set represents approximately one in twenty (5\% sample) of all the vehicles that traversed the extent of the corridor during this time period. Only vehicles that traverse the entire corridor present an opportunity to sample travel time. Vehicles that divert or stop for services along the corridor cannot be re-identified at the downstream station.

In the graph in Figure 1.1.2 one can distinguish the densest range of the travel time between 4-12 minutes as indicated by the density of the point cloud data. Travel time routinely extends to as high as 20 minutes or more. Little information beyond these general observations can be extracted about the general operating characteristics in the corridor. [Note that the occasional gap in the data is a result of equipment or communications failure, and is not a traffic phenomenon.]

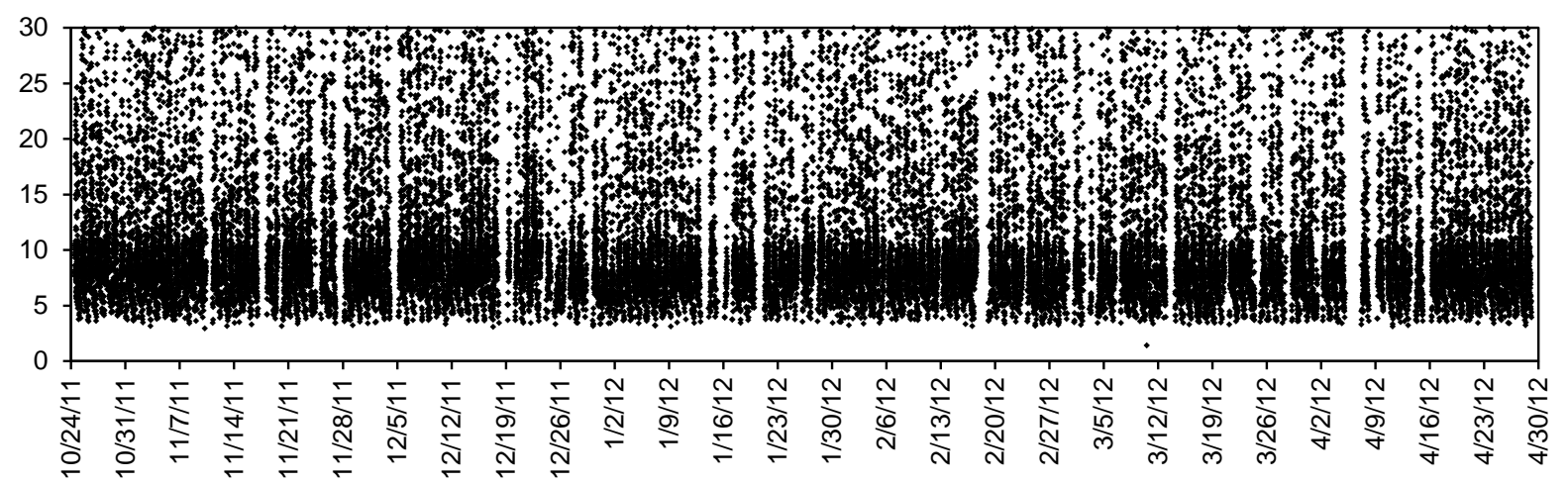

Figure 1.1.2 Vehicle travel time data for MD-355 over a 6-month time period

Data from a single day is shown in Figure 1.1.3. It depicts northbound travel time observations on Wednesday, 10/26/2011. For reference, the times at which the time-of-day signal timing changes are implemented are shown in red vertical dashed lines. These changes occur at 06:00, 09:30, 15:00, 19:00, and 22:00 hours. Although more detail is visible on the single-day plot than on the six month plot above, the ability to discern significant traffic trends is still lacking.

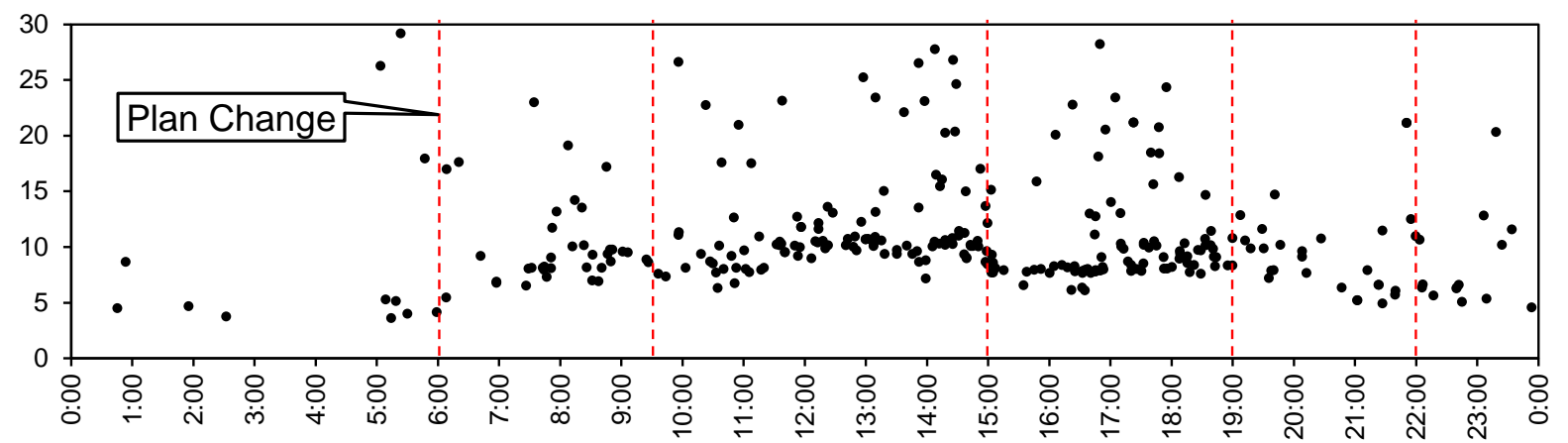

Figure 1.1.3 Vehicle travel-time data for MD-355 on Wednesday, 10/26/2011 
Figure 1.1.4 shows an example of the overlay technique for a 24 hour period. Each data point in this graph is the travel time from one vehicle that was matched between the entry and exit points of the arterial on any Wednesday over the six month period. Travel times generally fall within 5 and 15 minutes, varying by time of day. Some data points (such as those in callout $a$ ) indicate substantially longer travel times than the majority of vehicles. Such data points represent motorists who briefly left the road (for example, at a gas station) in route to their destination. As in Figure 1.1.3, the red vertical lines show the change in the signal timing at different times of day. Note that the travel time patterns change substantially at the transition of signal timing plans. This is most visible at 15:00 hours. Vehicle travel times appear to cluster around specific travel times, rather than being normally or uniformly distributed. The clustering of travel time creates what appears as horizontal stripes, or striations, in the data (see callout $b$ ). The time difference between the center of these horizontal stripes reflects the cycle length used in the corridor. As traffic builds in the evening rush between 15:00 and 19:00 hours, travel times escalate. At the beginning of the evening peak period most of the vehicles progress through in six minutes. At 17:00 hours, the majority shift to the second striation of approximately 8 minutes, and close to the end of the rush hour, a significant portion of vehicles progress through in about 10 minutes. This progression to longer travel times as the rush hour progresses indicates more vehicles are stopped and forced to wait for one or more signal cycles somewhere within the corridor.

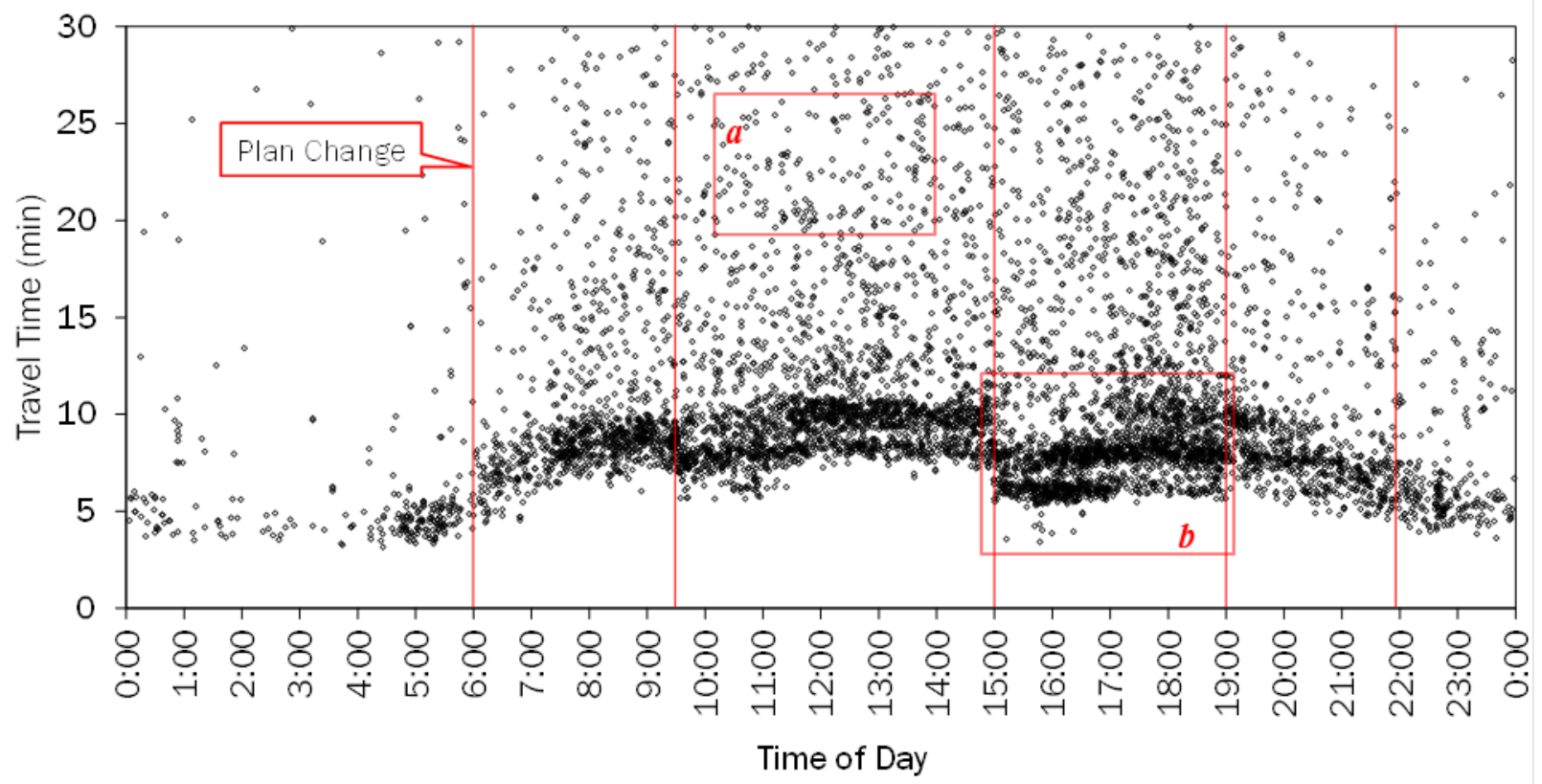

Figure 1.1.4 Travel times from vehicle re-identification displayed as a 24-hour overlay 
Overlay plots are useful to observe travel time trends for a single segment or corridor. Patterns in the point cloud data provide insight into the operations of the signal system and quality of traffic flow. Information that can be observed in overlay plots includes:

- Anticipated travel time through the corridor at various hours of the day

- $\quad$ Signal plan changes

- Cycle lengths (distance between striations)

- Mismatched cycles lengths within the corridor

- Proportion of people stopping for services within the corridor as indicated by the propensity of outliers

Although considerable insight can be gained from overlay plots, their utility is limited to observing a single corridor. Comparing the performance of multiple corridors, or performance of the same corridor at different dates or different times is not efficient with overlay charts.

Figure 1.1.5 shows the distribution of travel times observed during the AM period from 06:00 to 09:30 hour, which includes approximately 5,000 samples. Any travel time above 16.5 minutes has been removed, assuming that these travel times primarily result from vehicles stopping for services in the corridor and thus are not representative of the overall traffic patterns. The data for this AM period is portrayed as a histogram, in the vertical blue bars in Figure 1.1.5, and as a cumulative frequency distribution (CFD), in the bold black curve. The number of observations for each travel time in the histogram is shown on the right axis. The cumulative frequency of travel time is plotted in black as a single line and its value is shown on the left axis. Any point on the CFD provides a measure of percentage of vehicles that traverse the corridor within a known travel time, specifically travel time percentiles. For example, in the sample graph, 0.25 (or $25 \%$ ) traverse the corridor in 7.5 minutes or less, such that the $25^{\text {th }}$ percentile travel time is approximately 7.5 minutes. Note that all the information pertaining to the repeatable traffic pattern between $6 \mathrm{AM}$ and 9:30 AM in Figure 1.1.4, and also portrayed in the histogram in figure 1.1.5, is encoded into a single CFD curve.

The shape of the CFD curve in Figure 1.1.5 is typical for a corridor not experiencing congestion resulting in phase failures. The shape of the curve is referred to as an 's' curve or sigmoid-shaped curve. An 's' shaped curve can be modeled as a Gaussian (or normal) distribution with an appropriate mean and standard deviation. 


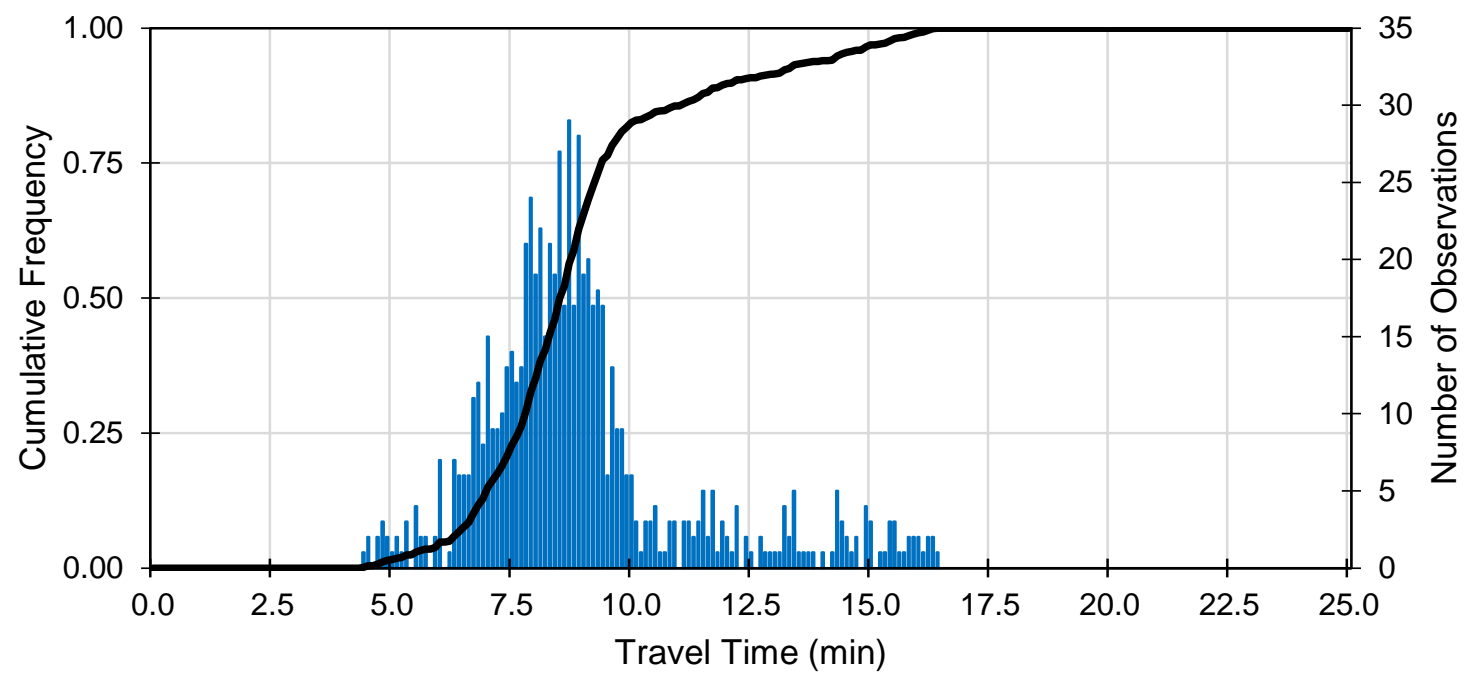

Figure 1.1.5 Distribution of Wednesday AM travel times on MD-355

Figure 1.1.6 shows the distribution of travel times observed on northbound MD 355 during the PM timing plan (15:00-19:00) on the same roadway. As with the AM data set, any travel time above 16.5 minutes is omitted, and the data is portrayed as a histogram and as a cumulative frequency diagram (CFD). During the PM peak period, large proportions of the vehicle traffic stream are forced to wait additional signal cycles to progress through the corridor, resulting in travel times clustered about 6.0, 8.0 and 10.0 minutes. The histogram in Figure 1.1.6 has at least three distinct peaks (also called modes), in contrast to a single peak in Figure 1.1.5. The multiple peaks (referred to as multi-modal data) of the data yield a CFD with multiple inflection points, as opposed to a single inflection point with an 's' shaped curve.

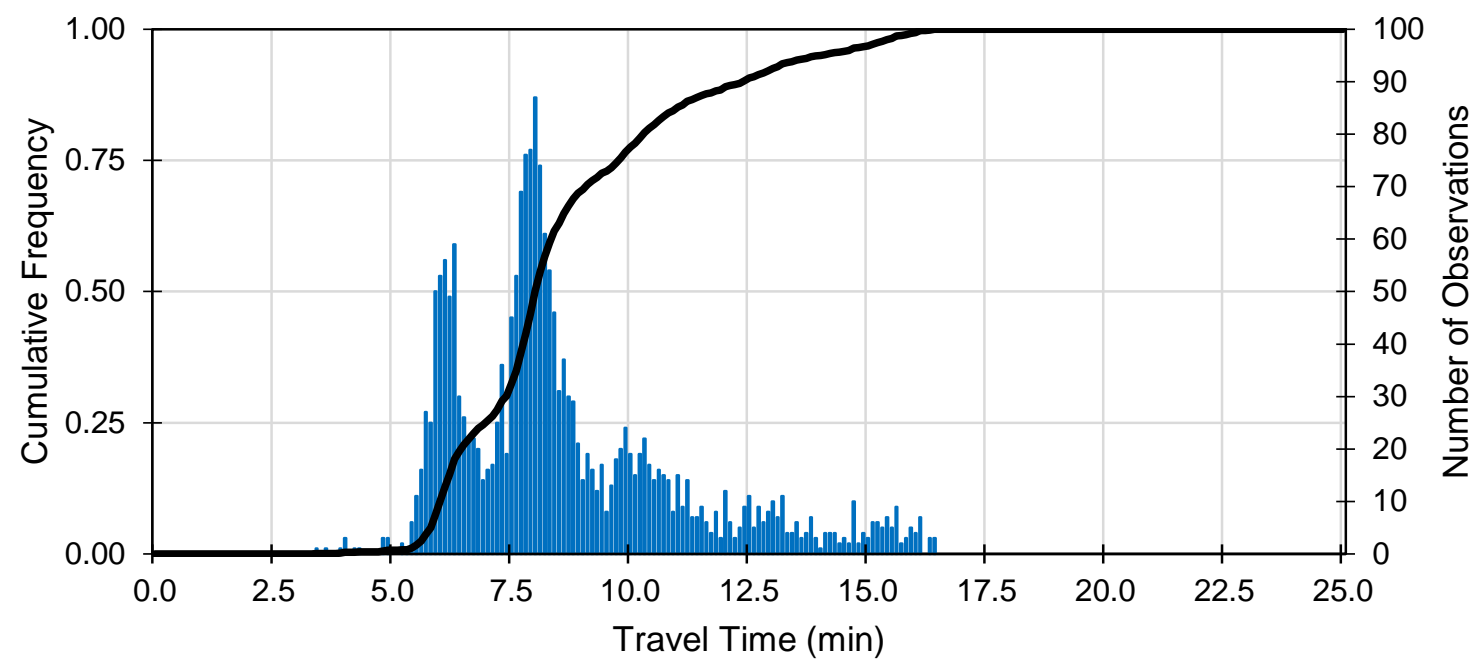

Figure 1.1.6 Distribution of Wednesday PM travel times on MD-355 
Cumulative frequency diagrams (CFDs) are useful to compactly portray the variation in travel time for a corridor using a single line. Characteristics of the distribution, such as uni-modal or multi-modal, are encoded in the shape of the curve. As a result, CFDs are suitable to compare travel time trends for multiple segments or multiple time periods on a single chart, providing not only the magnitude of travel times, but also an indication of the distribution of travel times for all the vehicles that traverse the corridor.

\subsection{Variation of Travel Time on Arterials Using Overlay and CFD Charts}

The following series of overlay charts and cumulative frequency diagrams (CFDs) was drawn from an archive of data collected as part of the Vehicle Probe Project validation program, sponsored and funded by the I-95 Corridor Coalition. From 2013 forward, the Coalition investigated the quality of outsourced probe data on numerous signalized corridors along the east coast, comparing the traffic patterns reported by probe data against a reference data set collected with Bluetooth traffic monitoring re-identification equipment. The resulting archive of re-identification data provides a rich source to illustrate various traffic performance phenomenon observable with re-identification travel time data. Most of the data sets from the validation effort span a two week period. The resulting overlay plots and CFDs reflect signalized arterial traffic performance on weekdays during the two weeks of data collection. Data was filtered using standard methods as described in the BluSTATs [2] processing manual.

For each example, a brief description of the roadway, including its location, physical and geometric attributes, and density of signal control, is provided. Most segments are from one to two miles in length, consistent with the validation procedures of the Coalition. The results of the comparison of reidentification with probe data are provided in a Coalition report [3], available at the Coalition web site. The examples of travel time data in this and the following sections are grouped by the common patterns that can be observed. The most common traffic or signal phenomena are provided first, and the less common phenomena are later. 
Figure 1.2.1 provides an example of a multi-modal travel distribution on an arterial roadway. The roadway segment is along New Jersey Route 42, southbound between the intersection of Tuckahoe Road and CR-698. The geometric attributes are provided in Table 1.2.1 below.

Table 1.2.1. Geometric properties of example segment NJ11-21 from NJ-42 southbound

\begin{tabular}{|c|c|c|c|c|c|c|c|c|}
\hline \multirow{4}{*}{ 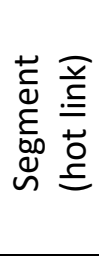 } & \multicolumn{6}{|c|}{ GEOMETRIC DESCRIPTION } & \multicolumn{2}{|c|}{ TMC CODES } \\
\hline & Crossroads & Lanes & AADT & Signals & & & \multirow[b]{2}{*}{ Begin } & \multirow[b]{2}{*}{ Len } \\
\hline & Starting at & Min & Min & $\#$ & $\begin{array}{l}\text { Access } \\
\text { Points }\end{array}$ & $\begin{array}{c}\text { Median } \\
\text { Barrier }\end{array}$ & & \\
\hline & Ending at & Max & Max & \#/Len & $\begin{array}{c}\text { Speed } \\
\text { Limit }\end{array}$ & $\begin{array}{c}\text { Major } \\
\text { Junctions }\end{array}$ & End & $\#$ \\
\hline \multirow{2}{*}{$\underline{21}$} & Tuckahoe Rd/Stagecoach Rd & 2 & 53581 & 2 & 28 & Yes & 103N09625 & 0.96 \\
\hline & CR-689/Cross Keys Berlin Rd & 2 & 53581 & 2.1 & 50 & 0 & $103-09624$ & 2 \\
\hline
\end{tabular}

Between 15:00 and 19:00 hours, this arterial exhibits two distinct travel times, one at approximately 70 seconds, and the other at approximately 140 second (see callout $i$ ). Travel times at other periods of the day do not exhibit this bi-modal distribution, and exhibit a mean travel time of approximately 110 seconds. The CFD plot to the right highlights the travel time CFD curve for the 17:00 to 18:00 hour timeframe in black (see callout ii). Its shape departs from the classic 's' curve due to its bi-modal properties, having two distinct bends, or knees. The inflection points on the bi-modal curve corresponding to the steepest points of ascent estimate the center of the two distinct travel time patterns (70 seconds and 110 seconds respectively). These are marked by the yellow dots. The portion of the traffic that traverses the corridor at each of the two travel time modes can be estimated by observing the percentiles on the $y$ axis. For the highlighted hour between 17:00 and 18:00 hours, approximately 30\% of traffic traverses the corridor at the faster mode (see callout iii), and approximately 55\% percent traverse the corridor between 90 seconds and 2.5 minutes which is the slower mode (see callout iv). The bi-modal curve appears as a combination of two classic 's' shaped curves (or sigmoid curves), as highlighted in callouts iii and iv, joined at an inflection point where the slope is a minimum. The minimum slope inflection point corresponds to the gap between the two dominant travel times; these represent corridor travel times that are unlikely due to signal timing.
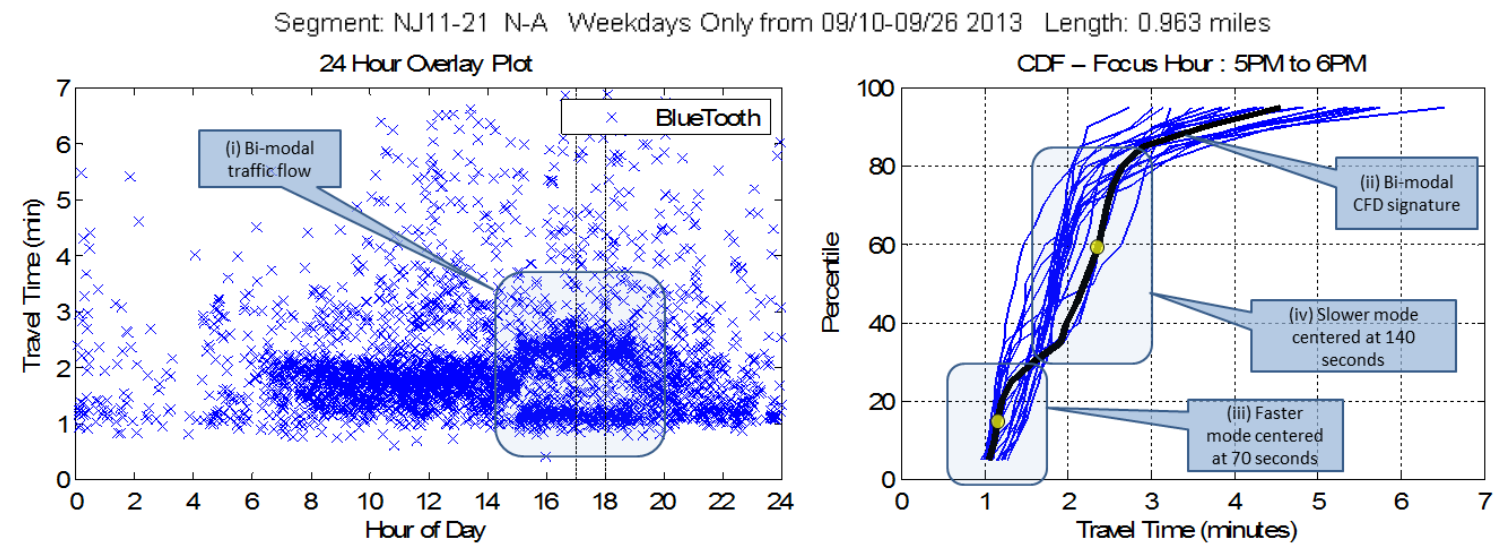

Figure 1.2.1. Overlay and CFD visualization of travel times for example segment on NJ-42 
Figure 1.2.2 provides another example of a multi-modal travel distribution on an arterial roadway. The roadway segment is along US-130, northbound in New Jersey between the intersection of CR-629 and NJ-413. The geometric attributes are provided in Table 1.2.2 below.

Table 1.2.2. Geometric properties of example segment NJ11-27 from US-130 northbound

\begin{tabular}{|c|c|c|c|c|c|c|c|c|}
\hline \multirow{4}{*}{ 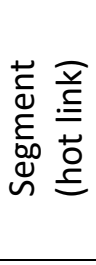 } & \multicolumn{6}{|c|}{ GEOMETRIC DESCRIPTION } & \multicolumn{2}{|c|}{ TMC CODES } \\
\hline & Crossroads & Lanes & AADT & Signals & & & \multirow[b]{2}{*}{ Begin } & \multirow[b]{2}{*}{ Len } \\
\hline & Starting at & Min & Min & $\#$ & $\begin{array}{l}\text { Access } \\
\text { Points }\end{array}$ & $\begin{array}{c}\text { Median } \\
\text { Barrier }\end{array}$ & & \\
\hline & Ending at & Max & Max & \#/Len & $\begin{array}{c}\text { Speed } \\
\text { Limit }\end{array}$ & $\begin{array}{c}\text { Major } \\
\text { Junctions }\end{array}$ & End & $\#$ \\
\hline \multirow{2}{*}{$\underline{27}$} & CR-629/Levitt Pkwy & 3 & 41989 & 5 & 40 & Yes & $103+05934$ & 2.03 \\
\hline & NJ-413/Keim Blvd & 3 & 41989 & 2.5 & 50 & 0 & $103+05934$ & 1 \\
\hline
\end{tabular}

In contrast to segment NJ11-21 from the previous example, this segment exhibits a bi-modal traffic pattern for a large majority of the day, beginning at 06:00 and lasting through 19:00 hours (see callout $i$ ). Even after 19:00 hours, a slight bi-modal pattern is discernable in the overlay plot, however a large majority of vehicles that traverse the corridor after 19:00 hours do so at the faster of the two modes. The CFD plot to the right highlights the $1 \mathrm{PM}$ to $2 \mathrm{PM}$ traffic in black in comparison with the ensemble of the CFDs for the other hours of the day. The bi-modal curve, appearing as a conjoined double 's' shaped curve, is characteristic of all the CFDs for the entire day, not just for the highlighted hour. The differences in the CFD for each hour are primarily vertical shifts in the curve. The inflection points for each hourly curve are roughly the same on the $x$ axis, indicating the same dominant travel pattern exists throughout the day. The locations of the inflection points vary primarily with respect to the $y$ axis, indicating that the portion of traffic that traverses the corridor at the faster mode versus the slower mode varies throughout the day. The magnitude of the variation is roughly $20 \%$ (see callout iii).
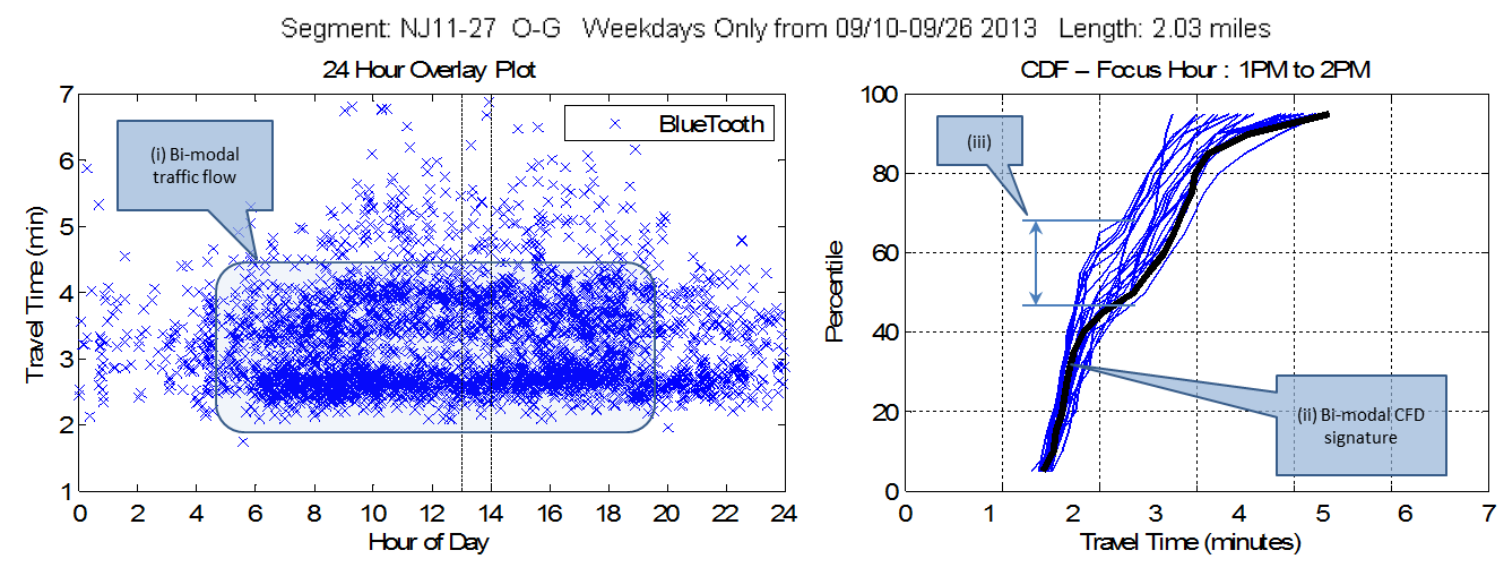

Figure 1.2.2 Overlay and CFD visualization of travel times for example segment on US-130 
Figure 1.2.3 provides yet another example of a multi-modal travel distribution on an arterial roadway. The roadway segment is along US Route 29 in northern Virginia, also known as Lee Highway, from the intersection with VA-123 to Jermantown Road. The geometric attributes for this segment are provided in Table 1.2.3 below.

Table 1.2.3 Geometric properties of example segment VA08-06 from US-29 southbound

\begin{tabular}{|c|c|c|c|c|c|c|c|c|}
\hline \multirow{4}{*}{ 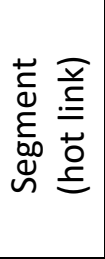 } & \multicolumn{6}{|c|}{ GEOMETRIC DESCRIPTION } & \multicolumn{2}{|c|}{ TMC CODES } \\
\hline & Crossroads & Lanes & AADT & Signals & & & \multirow[b]{2}{*}{ Begin } & \multirow[b]{2}{*}{ Len } \\
\hline & Starting at & Min & Min & $\#$ & $\begin{array}{l}\text { Access } \\
\text { Points }\end{array}$ & $\begin{array}{c}\text { Median } \\
\text { Barrier }\end{array}$ & & \\
\hline & Ending at & Max & Max & \#/Len & $\begin{array}{l}\text { Speed } \\
\text { Limit }\end{array}$ & $\begin{array}{c}\text { Major } \\
\text { Junctions }\end{array}$ & End & $\#$ \\
\hline \multirow{2}{*}{$\underline{06}$} & VA-123/Chain Bridge Rd & 2 & 35366 & 7 & 37 & Partial & $110+05739$ & 1.41 \\
\hline & Jermantown Rd & 2 & 37548 & 5.0 & 35 & 1 & $110+06793$ & 3 \\
\hline
\end{tabular}

This segment is unusual in that four distinct travel times, or modes, are visible in the overlay plot during the course of the day as seen in Figure 1.2.3. These modes are labeled in callouts $i$ through iv as the travel time escalates from a low of approximately 3.5 minutes, through travel times of approximately 5.0, 7.0, and finally 11.0 minutes at different times during the day. At any particular time of day, traffic is distributed between two travel times (or modes). A distribution between two modes results from a portion of the traffic stream being unable to clear the queue on green, and being forced to wait an extra cycle. Throughout the day, four distinct modes are observable at various hours, but never more than two at any given time.

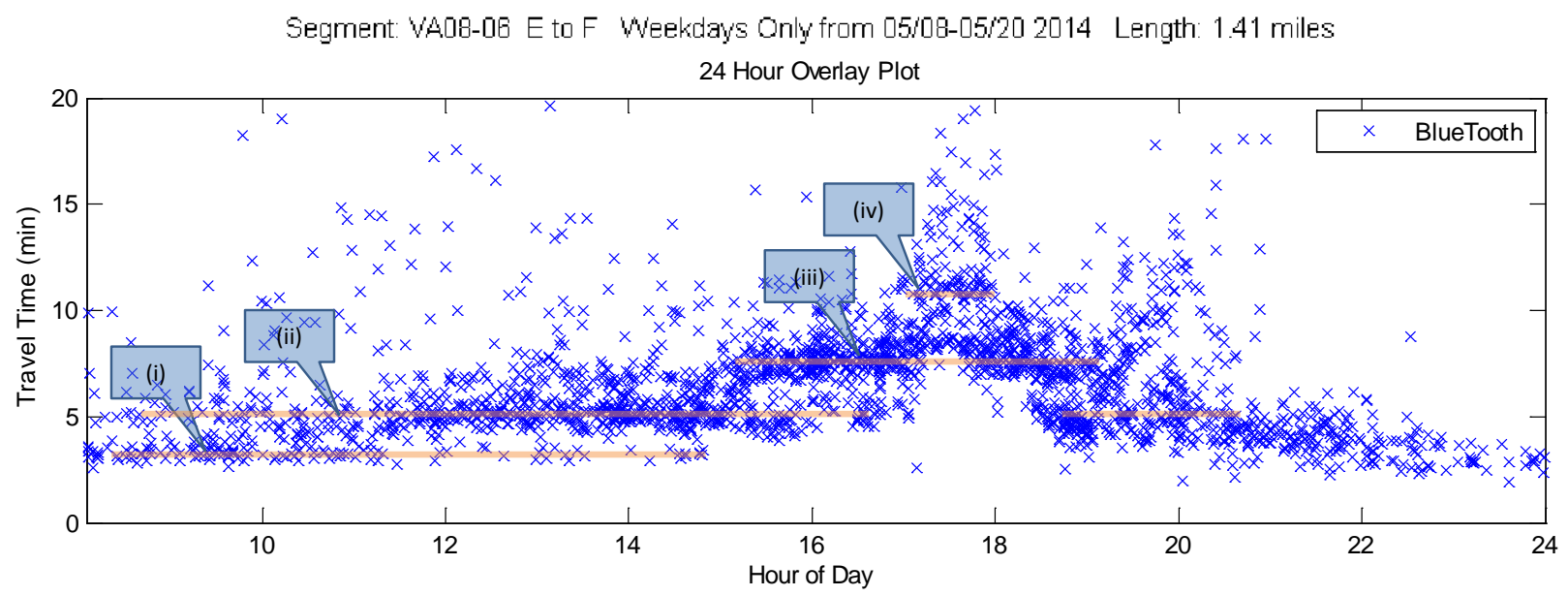

Figure 1.2.3 Overlay and CFD visualization of travel times for example segment on US-29 
The corresponding CFD for US-29 is shown in Figure 1.2.4. The peak evening rush hour is highlighted in black for the 17:00 to 18:00 hour (5 PM to 6 PM) timeframe. This CFD for this hour is a conjoined double 's' curve. Approximately half of the traffic progresses through at the faster mode (callout $i$ ), and the remaining at the slower mode (callout ii). Many of the CFD curves from other hours of the day shown in the diagram are also conjoined double ' $\mathrm{s}$ ' shapes, indicating bi-modal distribution. Unlike the previous example plot from US-130 in New Jersey, the inflection points that demark conjoined ' $\mathrm{s}$ ' curves vary horizontally as well as vertically, indicating that the travel time modes as well as their percent distributions vary throughout the day.

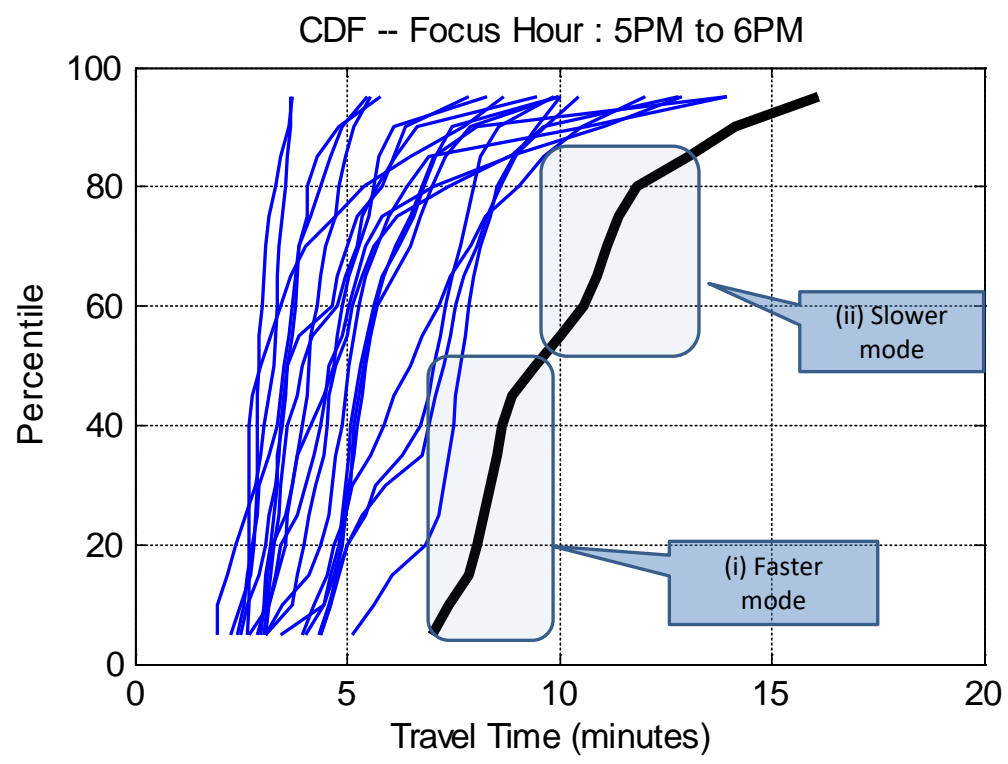

Figure 1.2.4 CFD visualization of travel times for example segment on US-29

Figure 1.2.5 provides another example of a multi-modal travel distribution on an arterial roadway, but the travel patterns are not as consistent from day to day as in other examples. The roadway segment is along US Route 29 in northern Virginia, also known as Lee Highway, from the intersection with Jermantown Road to VA-123. This is the same segment but opposite direction as the previous example. The geometric attributes of this segment are provided in Table 1.2.4 below.

Table 1.2.4. Geometric properties of example segment VA08-21 from US-29 northbound

\begin{tabular}{|c|c|c|c|c|c|c|c|c|}
\hline \multirow{4}{*}{ 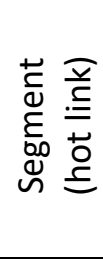 } & \multicolumn{6}{|c|}{ GEOMETRIC DESCRIPTION } & \multicolumn{2}{|c|}{ TMC CODES } \\
\hline & Crossroads & Lanes & AADT & Signals & & & \multirow[b]{2}{*}{ Begin } & \multirow[b]{2}{*}{ Len } \\
\hline & Starting at & Min & Min & \# & $\begin{array}{l}\text { Access } \\
\text { Points }\end{array}$ & $\begin{array}{c}\text { Median } \\
\text { Barrier }\end{array}$ & & \\
\hline & Ending at & Max & Max & \#/Len & $\begin{array}{c}\text { Speed } \\
\text { Limit }\end{array}$ & $\begin{array}{c}\text { Major } \\
\text { Junctions }\end{array}$ & End & $\#$ \\
\hline \multirow{2}{*}{$\underline{21}$} & Jermantown Rd & 2 & 35366 & 7 & 26 & Partial & $110-05739$ & 1.37 \\
\hline & VA-123/Chain Bridge Rd & 3 & 37548 & 5.1 & 35 & 1 & $110-05738$ & 3 \\
\hline
\end{tabular}


At least four distinct modes are visible between 06:00 and 10:00 hours. The pattern in Figure 1.2.5 is distinct from other examples in that all four modes are seen at the same time of day, from approximately 07:00 to 08:30. As previously discussed, for any given time period, traffic can only be distributed between two adjacent travel time modes, reflecting that a portion of traffic is able to progress through on green, and a portion is forced to wait an additional signal cycle. Multiple modes (greater than two) occurring at the same time frame in an overlay chart indicates significant variation in travel time from day to day. In the example shown, it is likely that the slower modes (those greater than eight minutes as noted by callouts iii and iv) were experienced on days with extreme congestion perhaps induced by an incident or road construction. Additional analysis is needed to resolve the underlying cause. One possible cause may be substantially different traffic patterns for different weekdays (for example Monday and Friday traffic may be substantially different than Tuesday, Wednesday and Thursday traffic). Another possible cause may be that event, construction activity, or maintenance activity was active for a portion of the data collection period. Lane closures for maintenance during portions of the data collection period may induce such patterns. An isolated traffic event, such as a traffic accident, typically does not produce such patterns in an overlay chart. The travel times for a traffic accident are unusually long, but are not repeated from day to day, and thus are not reinforced by the overlay chart. As such, accidents and events tend to appear as outlier data in overlay charts rather than alternate modes. The travel times associated with the faster modes in Figure 1.2.5, as indicated at callouts $i$ and ii, are visible at other times of the day, whereas the slower modes, callouts iii and iv, are only visible between 07:00 to 08:30. This may suggest that the slower modes may not be typical traffic patterns. Segregating data by day of week, or observing daily travel time plots may analyze the data appropriately.

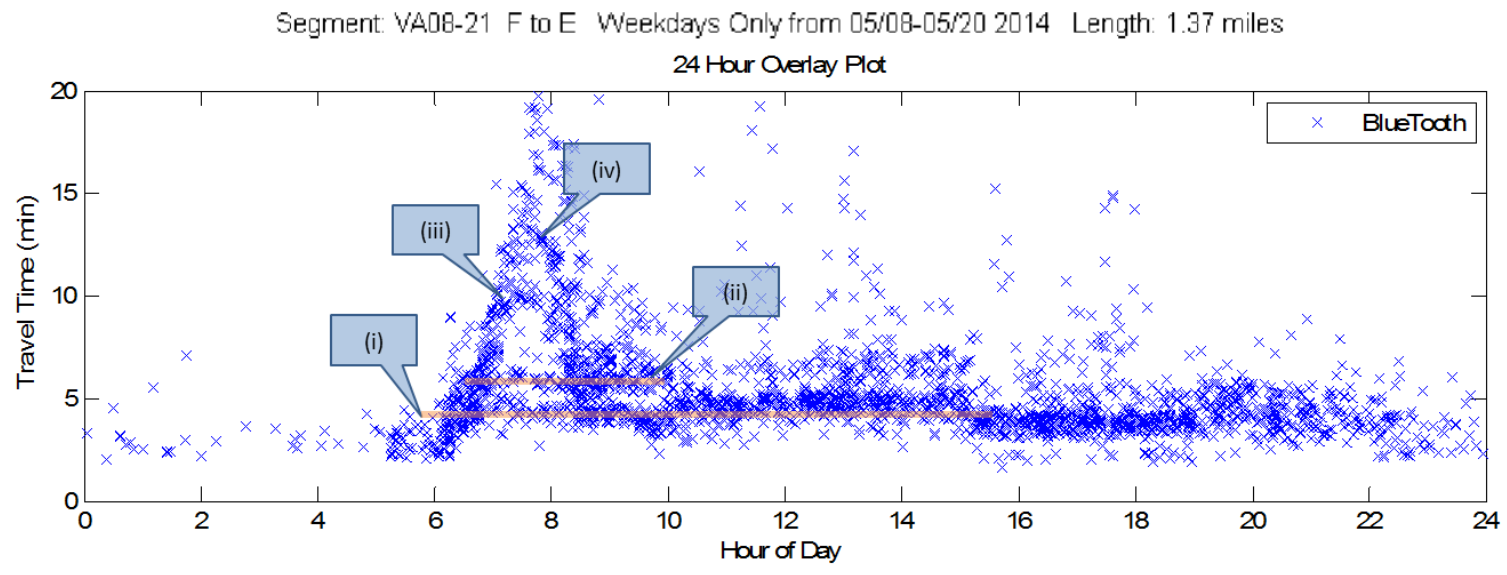

Figure 1.2.5 CFD visualization of travel times for example segment on US-29 northbound

\subsection{Signal Controller Attributes Observable in Overlay Charts}

As in section 1.2, sample plots that exhibit signal controller attributes are taken from the archive of arterial validation data collected as part of the I-95 Corridor Coalition's Vehicle Probe validation effort. These examples illustrate signal timing parameters that can be observed in travel time overlay charts. The three phenomena illustrated include signal timing changes, cycle length mismatches between adjacent signals within a corridor, and travel times on a cycle-by-cycle basis. 
Some signal timing changes (that is when timing plans change throughout the day) were visible in the examples used in section 1.2, however the signal timing changes were in combination with traffic signatures and were not the dominant feature in the overlay charts in the previous section. The examples below are chosen to isolate or magnify the characteristic signatures of signal timing changes apart from other traffic phenomena. Figure 1.3.1 provides an example of signal timing change that is distinctly visible in a weekday travel time overlay chart. The roadway segment is along US Route 130 in New Jersey, southbound between the intersection of Beverly Rancocas Road and CR-629. The geometric attributes for this segment are provided in Table 1.3.1 below.

Table 1.3.1 Geometric properties of example segment NJ11-26 from US-130 northbound

\begin{tabular}{|c|c|c|c|c|c|c|c|c|}
\hline \multirow{4}{*}{ 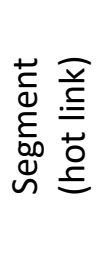 } & \multicolumn{6}{|c|}{ GEOMETRIC DESCRIPTION } & \multicolumn{2}{|c|}{ TMC CODES } \\
\hline & Crossroads & Lanes & AADT & Signals & & & \multirow[b]{2}{*}{ Begin } & \multirow[b]{2}{*}{ Len } \\
\hline & Starting at & Min & Min & $\#$ & $\begin{array}{l}\text { Access } \\
\text { Points }\end{array}$ & $\begin{array}{c}\text { Median } \\
\text { Barrier }\end{array}$ & & \\
\hline & Ending at & Max & Max & \#/Len & $\begin{array}{l}\text { Speed } \\
\text { Limit }\end{array}$ & $\begin{array}{l}\text { Major } \\
\text { Junctions }\end{array}$ & End & $\#$ \\
\hline \multirow{2}{*}{$\underline{26}$} & $\begin{array}{l}\text { Beverly Rancocas Rd/Mount } \\
\text { Holly Rd }\end{array}$ & 3 & 41989 & 3 & 28 & Yes & $103+05932$ & 1.47 \\
\hline & CR-629/Levitt Pkwy & 3 & 41989 & 2.0 & 50 & 0 & 103Р05933 & 3 \\
\hline
\end{tabular}

The signal timing change is visibly evident at 15:30 hours, at callout $i$. The dominant travel time shifts from approximately three minutes to two minutes. This is due to a signal timing plan implemented for PM peak that minimized travel time for northbound traffic. A less distinct signal timing change also occurs at approximately 18:30 (see callout ii), in which the dominant travel time shifts back to approximately three minutes.

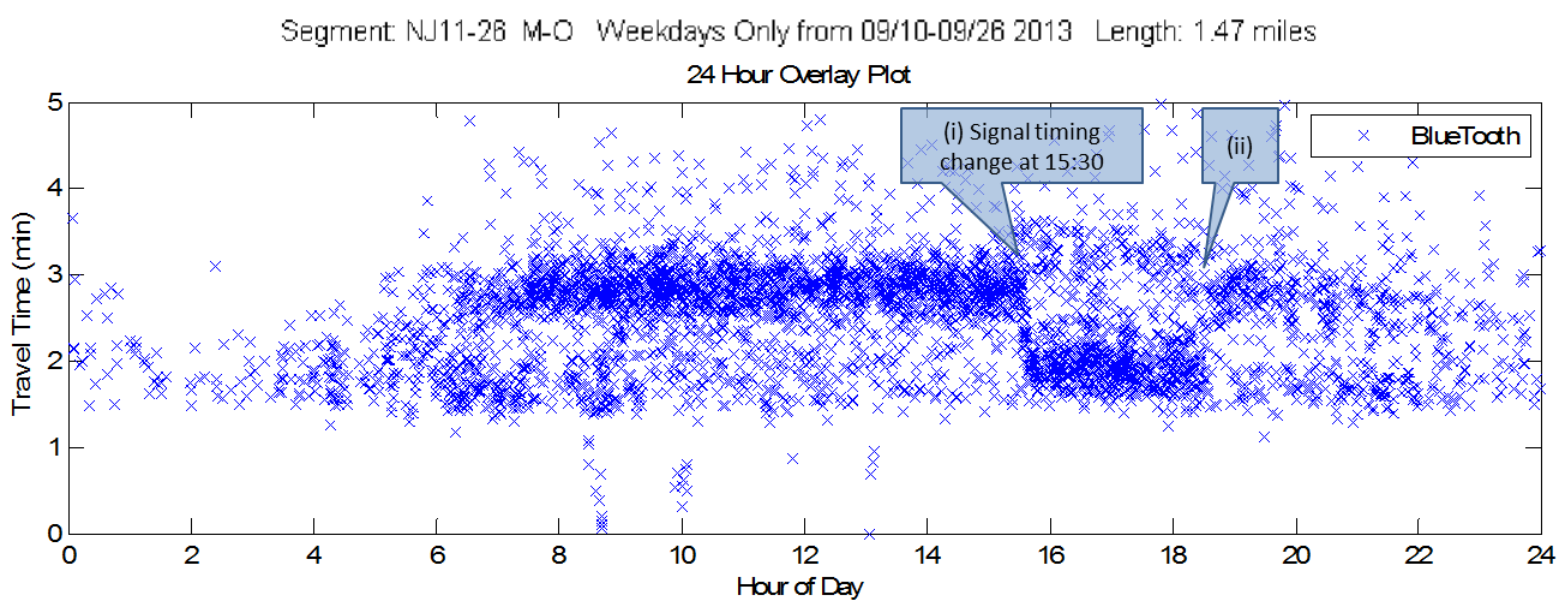

Figure 1.3.1 Overlay chart for US-130 northbound showing signal timing change 
Figure 1.3.2 provides another example of signal timing change that is distinctly visible in a weekday travel time overlay chart. The roadway segment is along New Jersey Route 42, northbound between the intersection of Fries Mill Road and NJ-168. The geometric attributes are provided in Table 1.3.2 below.

Table 1.3.2 Geometric properties of example segment NJ11-18 from NJ-42 northbound

\begin{tabular}{|c|c|c|c|c|c|c|c|c|}
\hline \multirow{4}{*}{ 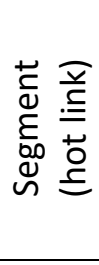 } & \multicolumn{6}{|c|}{ GEOMETRIC DESCRIPTION } & \multicolumn{2}{|c|}{ TMC CODES } \\
\hline & Crossroads & Lanes & AADT & Signals & & & \multirow[b]{2}{*}{ Begin } & \multirow[b]{2}{*}{ Len } \\
\hline & Starting at & Min & Min & $\#$ & $\begin{array}{l}\text { Access } \\
\text { Points }\end{array}$ & $\begin{array}{c}\text { Median } \\
\text { Barrier }\end{array}$ & & \\
\hline & Ending at & Max & Max & \#/Len & $\begin{array}{c}\text { Speed } \\
\text { Limit }\end{array}$ & $\begin{array}{c}\text { Major } \\
\text { Junctions }\end{array}$ & End & $\#$ \\
\hline \multirow{2}{*}{$\underline{18}$} & Fries Mill Rd & 2 & 53581 & 5 & 33 & Yes & 103Р09626 & 1.33 \\
\hline & NJ-168/Atlantic City Expy & 2 & 53581 & 3.8 & 45 & 0 & $103+04295$ & 3 \\
\hline
\end{tabular}

Signal timing changes are evident at 15:00 hours (at callout $i$ ), and again at 20:00 hours (at callout ii). On this segment of NJ-42, the entire distribution of travel times appears to shift by approximately one minute greater at 15:00 hours, and then shift to back one minute less at 20:00 hours. Abrupt changes in the travel time patterns such as those exhibited in Figures 1.3.1 and 1.3.2 are characteristic of signal timing changes.

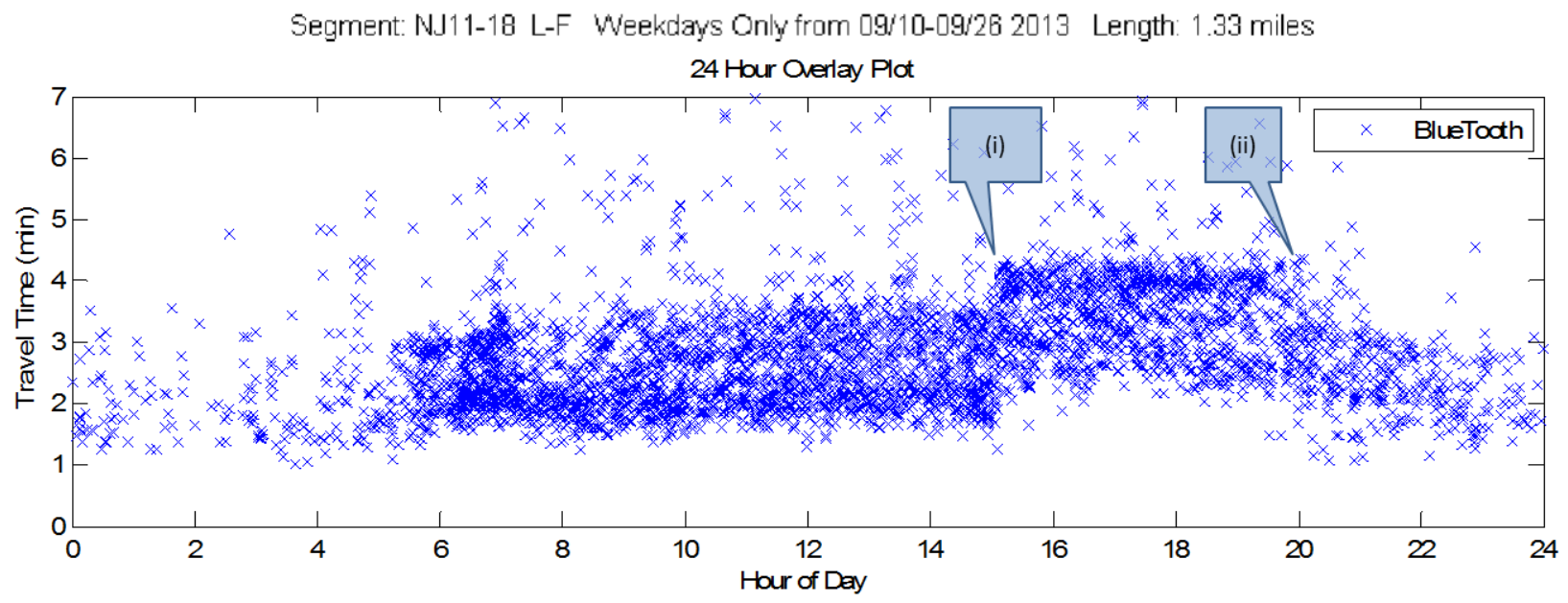

Figure 1.3.2. Overlay chart for NJ-42 northbound showing signal timing changes

Note that signal timing changes are observable in multi-modal examples used in section 1.2. An example of this is in Figure 1.2.5 near 15:00 hours in which the abrupt change in travel time can be attributed to a signal timing change. Also, in Figure 1.2.1, a signal timing change accompanies the transition of traffic flow into bi-modal flow (at around 15:00 hours) and out of bi-modal flow (at around 19:00 hours).

Corridors with coordinated signals operate on equal cycle lengths. If cycle lengths differ, even by a second or two, the platoons from an upstream signalized intersection will arrive at different points in the cycle of the downstream signalized intersection. The difference with each cycle will grow (or diminish) depending on the relative cycle length of adjacent intersections. Mismatched cycle lengths have a distinct pattern in overlay plots. Diagonal stripes (or striations) appear as a result. 
Figure 1.3.3 provides an example of mismatched cycle times within a corridor visible in a weekday travel time overlay chart. The roadway segment is along US Route 130 in New Jersey, northbound between the intersection of Bridgeboro Street and Beverly Rancocas Road. The geometric attributes of this segment are provided in Table 1.3.3 below.

Table 1.3.3. Geometric properties of example segment NJ11-25 from US-130 northbound

\begin{tabular}{|c|c|c|c|c|c|c|c|c|}
\hline \multirow{4}{*}{ 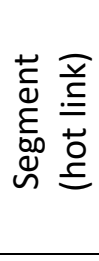 } & \multicolumn{6}{|c|}{ GEOMETRIC DESCRIPTION } & \multicolumn{2}{|c|}{ TMC CODES } \\
\hline & Crossroads & Lanes & AADT & Signals & & & \multirow[b]{2}{*}{ Begin } & \multirow[b]{2}{*}{ Len } \\
\hline & Starting at & Min & Min & $\#$ & $\begin{array}{l}\text { Access } \\
\text { Points }\end{array}$ & $\begin{array}{l}\text { Median } \\
\text { Barrier }\end{array}$ & & \\
\hline & Ending at & Max & Max & \#/Len & $\begin{array}{l}\text { Speed } \\
\text { Limit }\end{array}$ & $\begin{array}{c}\text { Major } \\
\text { Junctions }\end{array}$ & End & $\#$ \\
\hline \multirow[b]{2}{*}{$\underline{25}$} & Bridgeboro St & 3 & 41989 & 2 & 11 & Yes & $103 P 05930$ & 1.51 \\
\hline & $\begin{array}{c}\text { Beverly Rancocas Rd/Mount } \\
\text { Holly Rd }\end{array}$ & 3 & 41989 & 1.3 & 50 & 0 & 103Р05931 & 3 \\
\hline
\end{tabular}

The two signals on this corridor are operated on different cycle lengths. The diagonal striations in the data indicate the phenomenon. On each successive cycle, the platoon from the upstream signal arrives at a slightly different time in the downstream cycle causing the cyclic travel times patterns displayed in the overlay chart. This pattern is observable throughout the day, even as new signal timing plans are initiated near 15:45 and 18:30 hours. This pattern provides no indication if the mismatched cycle times were by design, as would be the case at the junction of two coordinated arterial corridors, or accidental, perhaps as a result of a data entry error. The consistency of the phenomenon in Figure 1.3.3 throughout the day suggests the former, whereas the striation pattern for only a portion of the day would suggest the latter.

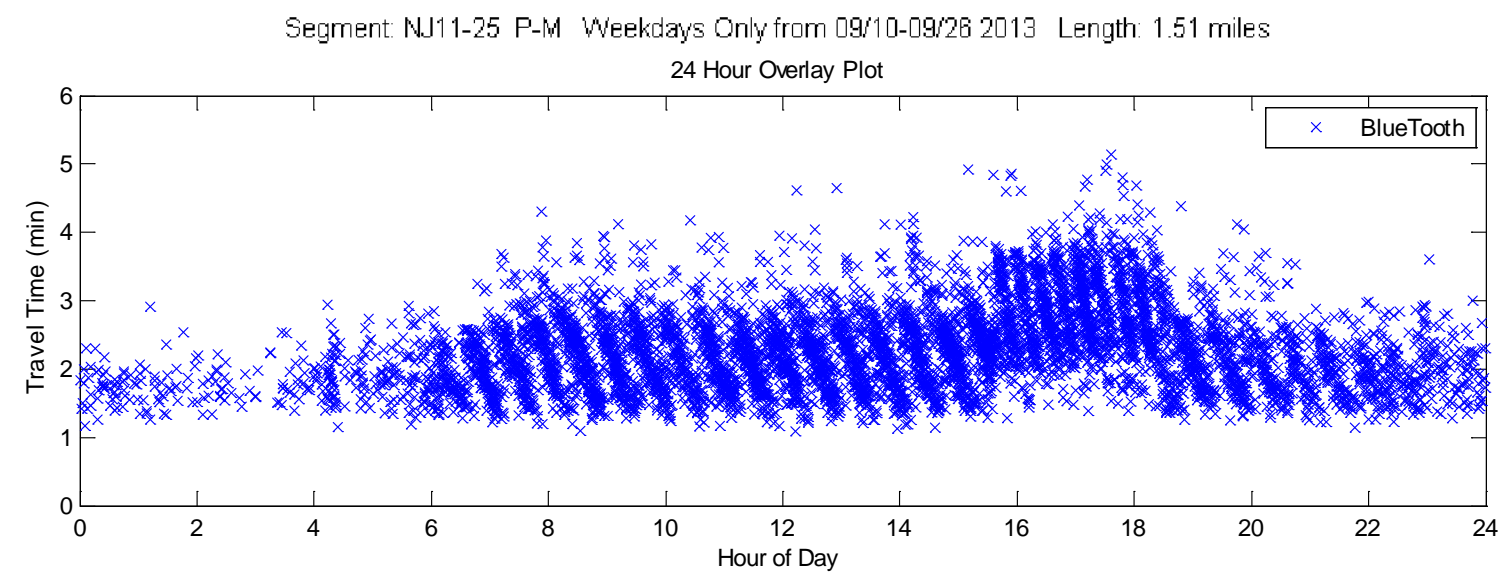

Figure 1.3.3 Overlay chart for US-130 segment northbound showing mismatched cycle lengths 
Figure 1.3.4 provides another example of mismatched signal cycle lengths along US Route 29 in northern Virginia, northbound between Gallows Road and Graham Road. The geometric attributes for this segment are provided in Table 1.3.4 below.

Table 1.3.4. Geometric properties of example segment VA08-26 from US-29 northbound

\begin{tabular}{|c|c|c|c|c|c|c|c|c|}
\hline \multirow{4}{*}{ 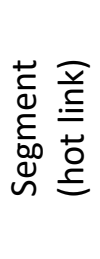 } & \multicolumn{6}{|c|}{ GEOMETRIC DESCRIPTION } & \multicolumn{2}{|c|}{ TMC CODES } \\
\hline & Crossroads & Lanes & AADT & Signals & & & \multirow[b]{2}{*}{ Begin } & \multirow[b]{2}{*}{ Len } \\
\hline & Starting at & Min & Min & $\#$ & $\begin{array}{l}\text { Access } \\
\text { Points }\end{array}$ & $\begin{array}{c}\text { Median } \\
\text { Barrier }\end{array}$ & & \\
\hline & Ending at & Max & Max & \#/Len & $\begin{array}{c}\text { Speed } \\
\text { Limit }\end{array}$ & $\begin{array}{c}\text { Major } \\
\text { Junctions }\end{array}$ & End & $\#$ \\
\hline \multirow{2}{*}{$\underline{26}$} & Gallows Rd & 2 & 28831 & 7 & 20 & Yes & 110N07955 & 1.93 \\
\hline & Graham Rd/Woodley PI & 3 & 28831 & 3.6 & 40 & 2 & $110-06788$ & 2 \\
\hline
\end{tabular}

Unlike the previous example, the striations are not as distinct. During the AM peak period between 6 AM and $10 \mathrm{AM}$, the telltale striations are visible, but not as distinct as the previous example segment on US130 in New Jersey which spanned only two signals. During the PM period on US-29, the striations are even less distinct, and could be easily overlooked if the striations observed in the AM period did not prompt close examination. Note that this sample segment from US-29 spans seven signalized intersections. As the number of signalized intersections increases, the clarity of characteristic data signatures diminishes, whether it is striations from mismatched signal cycle lengths, or multi-modal travel time distributions.

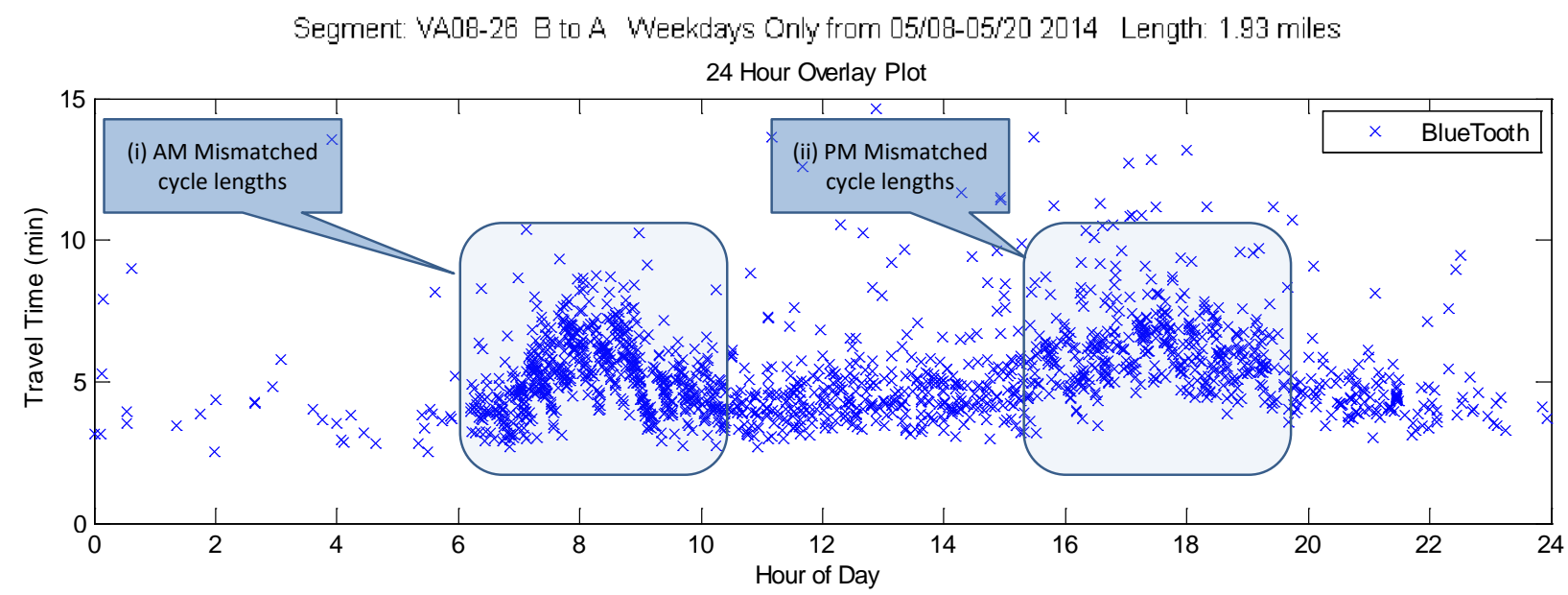

Figure 1.3.4 Overlay chart for US-29 segment northbound showing mismatched cycle lengths

Individual cycle operation is occasionally visible in overlay charts. This signature appears similar to the striations that characterize mismatched signal cycles, but is visible at small time scales as shown in Figure 1.3.5. This phenomenon was discovered only by accident while browsing overlay charts at high magnification. Over a multi-day period for fixed timed signals, as shown in Figure 1.3.5, travel times vary between approximately 5.2 and 7.5 minutes as a function of arrival time at the beginning of the segment. The variability in travel time is a function of when the vehicle arrives from the upstream intersection with respect to the corridors timing cycle. This creates a repeatable pattern of travel time as displayed by the 
visible stripes in the data (callout $i$ ). The time distance between the striations is equal to the cycle length (callout $i$ ).

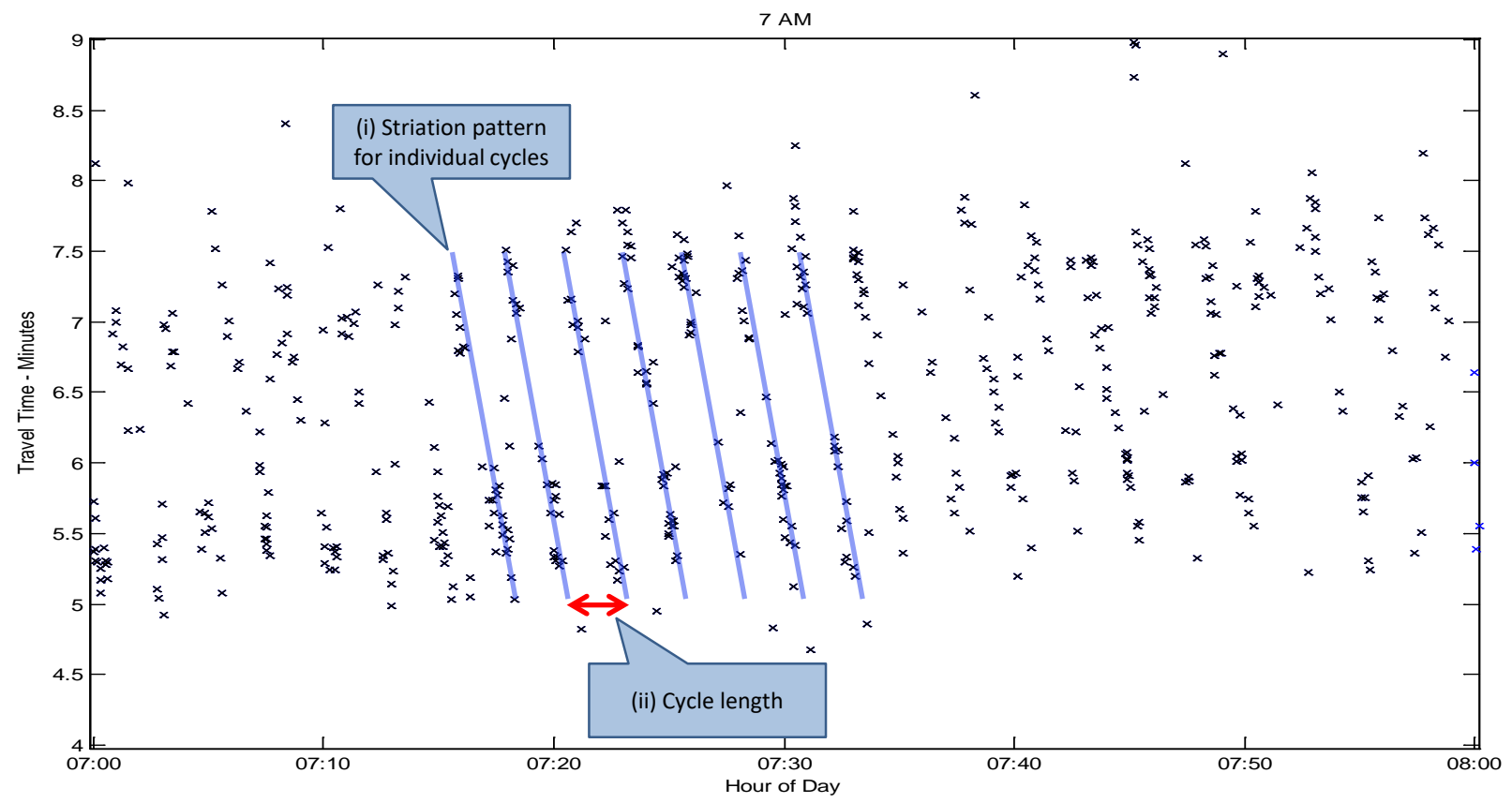

Figure 1.3.5 Overlay chart displaying individual cycle travel times

Note this data signature is not frequently visible. It requires fixed-timed signals with highly repeatable daily cycle times. If the number of signals in the corridor is large (greater than two), the dispersion in the data will likely mask any such patterns. Any type of adaptive or variable length offset will also cause dispersion in the data over several days, obscuring such patterns.

\subsection{Before and After Comparison with CFDs}

The construction of CFDs was covered in section 1.1, and their ability to portray characteristic multimodal travel time distributions were covered in section 1.2. However, the most useful attribute for CFD charts is their ability to compare traffic performance between two difference roadways, or at two different times on the same roadway, or traffic performance before and after signal retiming, or the impact on traffic due to a capital improvement on the corridor or network.

Comparing travel times using overlay charts is cumbersome, requiring the user to align time frames and interpret the relative density of data points. Measurements such as mean or median travel times compare only central tendencies, not the full distribution of travel time. Measures such as travel time index (TTI), buffer time index (BTI), standard deviation, or interquartile range (IQR) can characterize the magnitude of variation in the travel time, but reveal nothing of its underlying shape such as uni-modal versus bimodal. Cumulative frequency diagrams (CFDs) fully capture traffic performances in a compact, visual format, displaying not only the change in average conditions, but also any changes in travel time variation.

The first example of comparative CFDs portrays the traffic benefit attributed to a major capital improvement. Maryland Route 200 (MD 200), more commonly known as the Intercounty Connector or 
ICC, is a tolled freeway in Maryland which connects Gaithersburg in Montgomery County and Laurel in Prince George's County. Although opened in stages, the portion of the ICC that allowed for connection between I-270 and I-95 opened on November 22, 2011. Two re-identification sensors were installed on the impacted network prior to the opening of the ICC. One was on I-270, north of its intersection with the ICC (sensor A in Figure 1.4.1), and the other was on I-95 between Washington DC and Baltimore (sensor $B$ in Figure 1.4.1). Re-identification data was collected and analyzed before and after the opening of the ICC. Travel times derived from re-identification sensor A and B reflect trips between Montgomery County and Baltimore, Maryland.

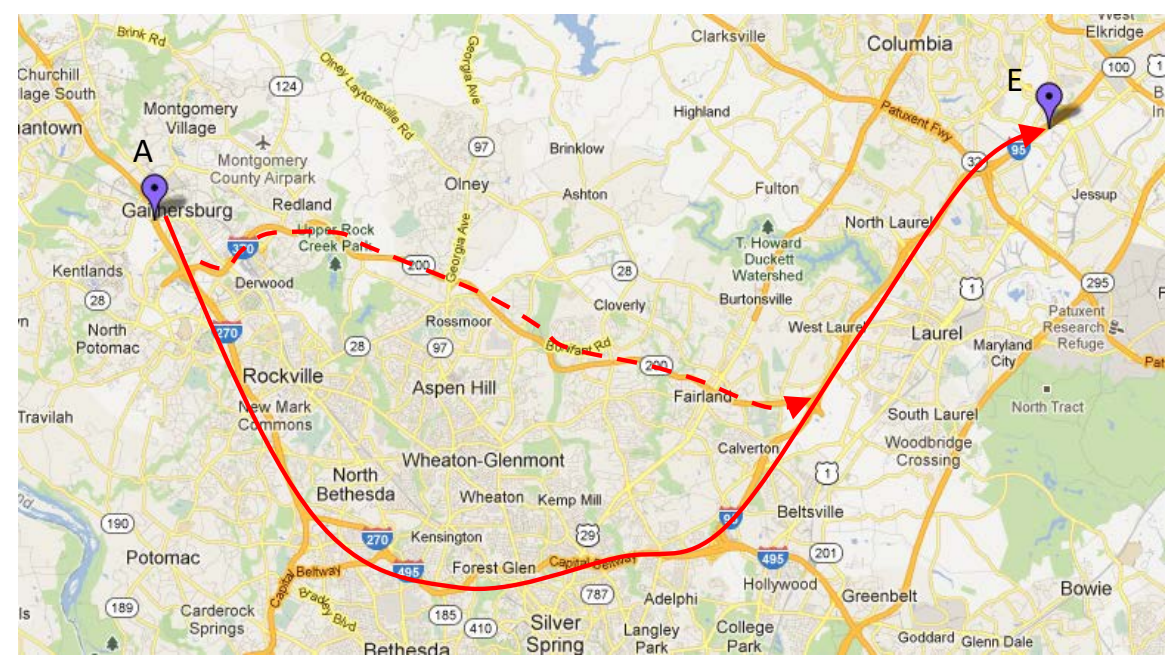

Figure 1.4.1 The Inter-County Connect (MD-200) in Maryland

Even though no re-identification sensor was located directly on the ICC, sensors A and B captured the impact due to the opening of the ICC on travelers from Montgomery County to Baltimore, whether they accessed the ICC (dashed lined) or used the non-tolled route that included portions of the DC beltway (solid line). The before/after comparison of the CFDs are shown in Figure 1.4.2. Not only did the travel time lessen as indicated by the shift from right to left in the respective CFDs (see callout $i$ ), it also became more reliable as evidenced by shift of the curve up and to the left (see callout ii). 


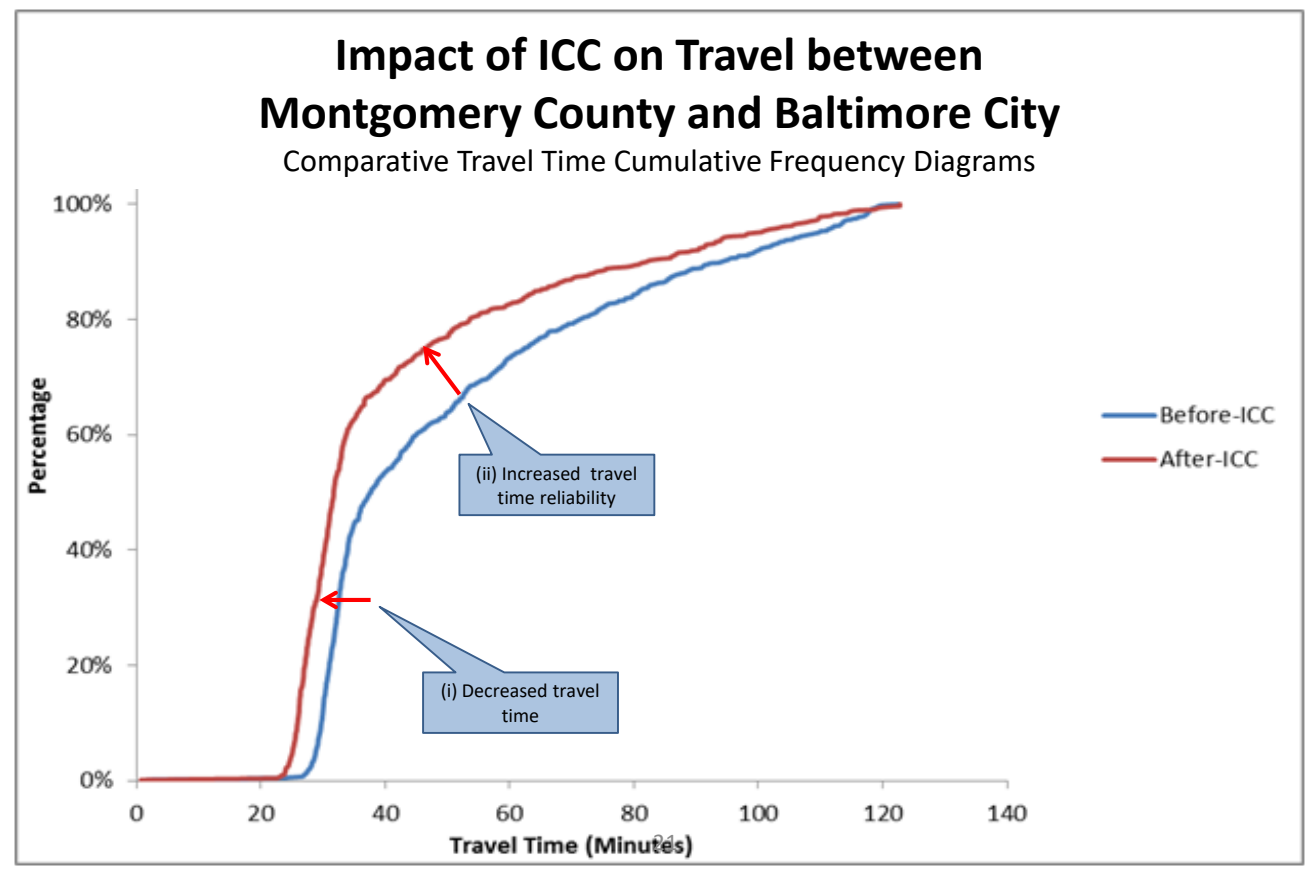

Figure 1.4.2. Travel time impact of the ICC

The second example of the use of comparative CFDs reveals the improvement resulting from signal retiming. In February of 2009, Bluetooth re-identification sensors were deployed at various locations on Maryland Route 24 north of Baltimore near the intersection with Interstate 95, as shown in Figure 1.4.3. The sensors were placed to measure the impact of signal retiming.

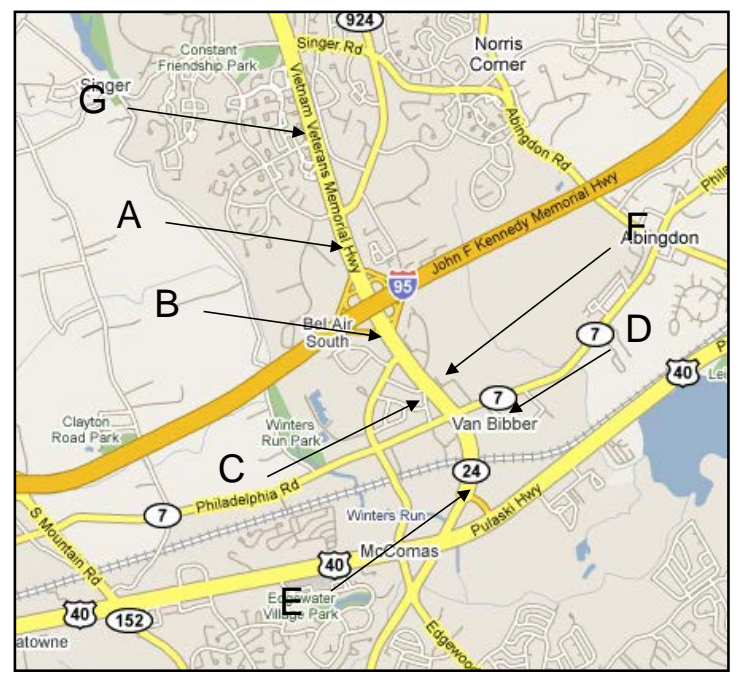

Figure 1.4.3 Location of Bluetooth sensors on Maryland 24 
Travel time data was for captured 2.5 days before and two days after implementation of a new signal timing plan. The before and after travel time histograms in Figure 1.4.4 illustrate the effectiveness of the arterial signal retiming for northbound traffic during the PM peak between sensors $\mathrm{E}$ and $\mathrm{A}$. The shift of the histogram to the left indicates a reduction in travel time. The same data is portrayed in Figure 1.4.5 using comparative CFDs, providing a compact and succinct view of the impact of signal retiming on the travel time distribution.

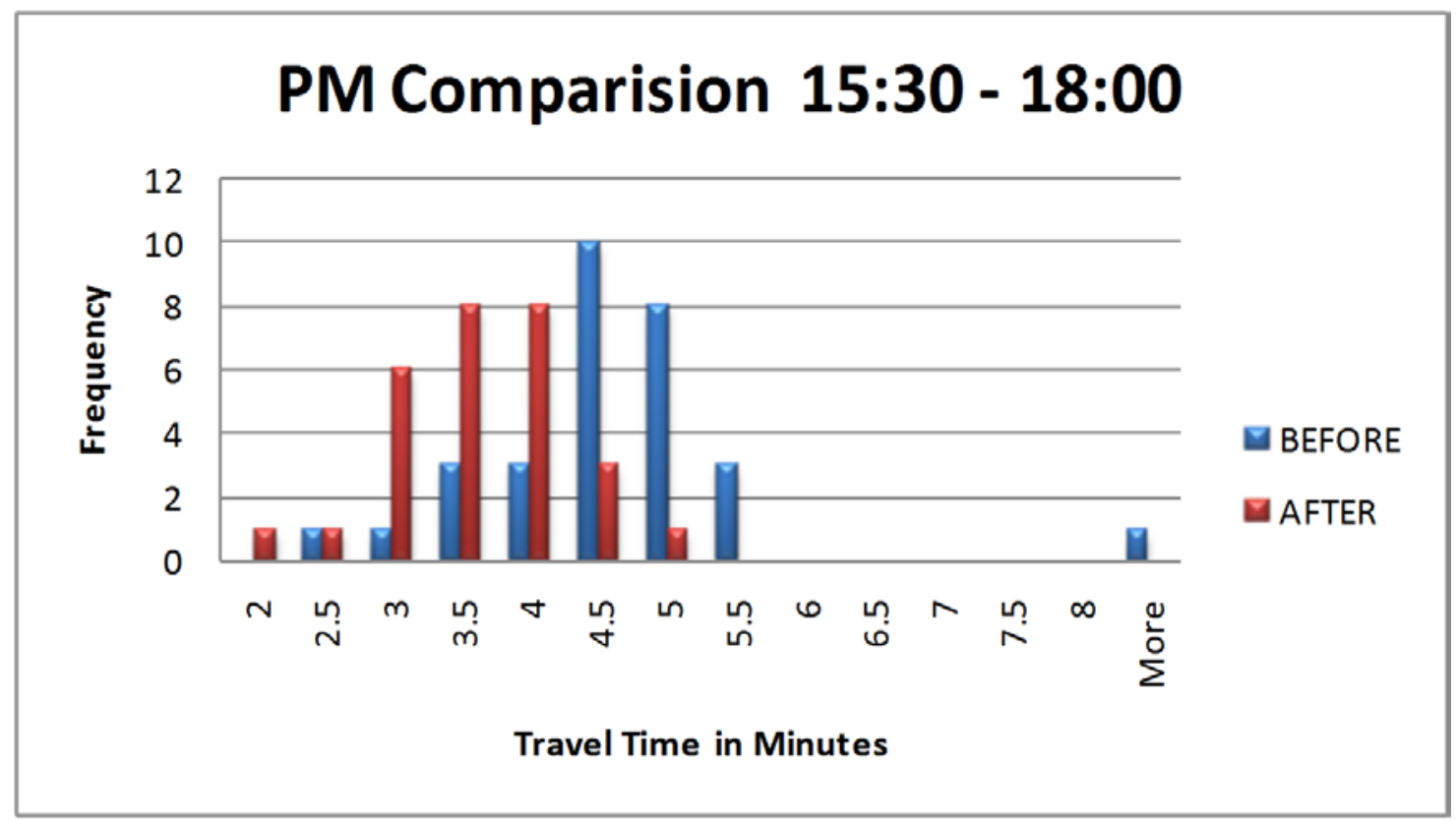

Figure 1.4.4 Before and after histogram of travel times on Maryland Route 24

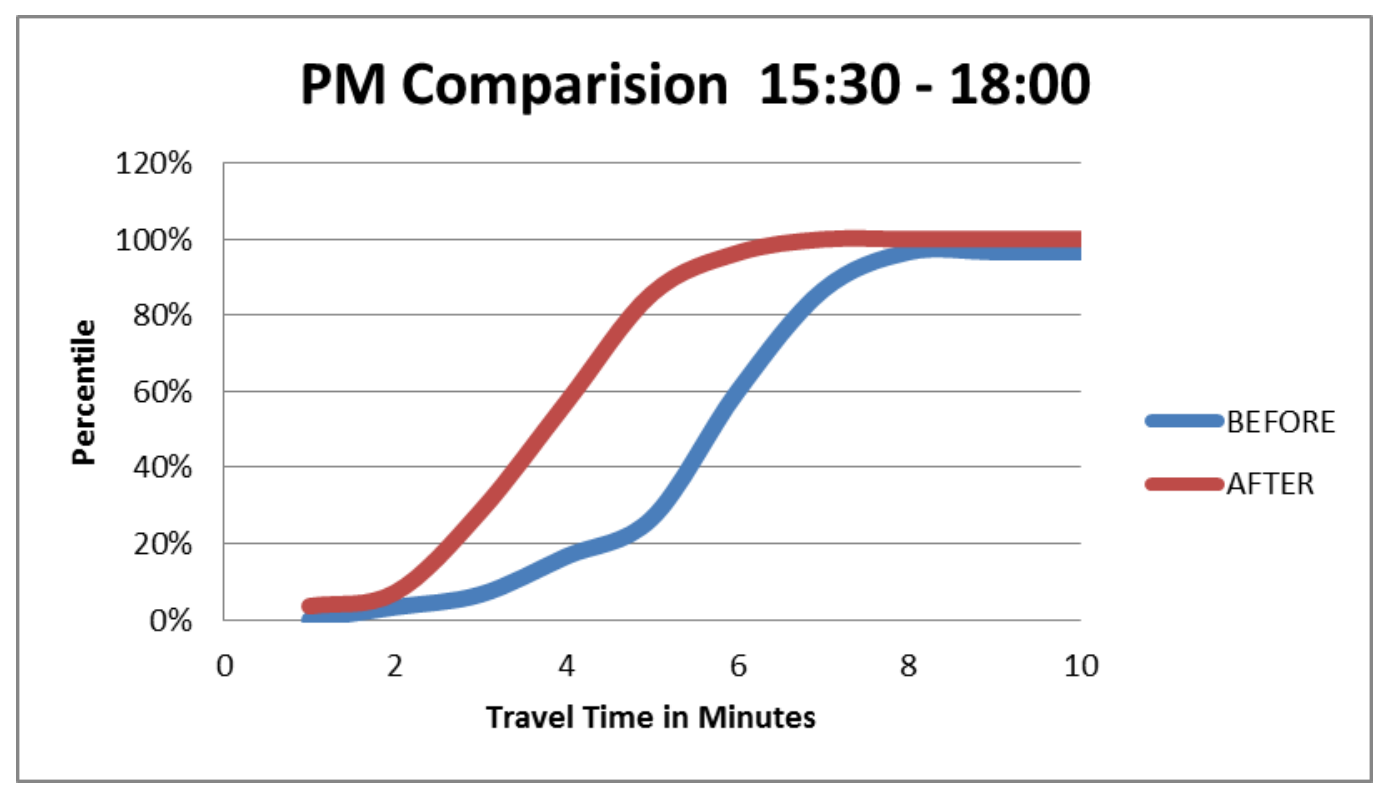

Figure 1.4.5 Before and after comparative CFD of travel times on Maryland Route 24 
The last example of comparative CFDs is to examine the year to year performance changes on an arterial segment. This data sample comes from a roadway that begins on Maryland route 355 (Rockville Pike), at the intersection of Country Club Road, and ends at the intersection of $1^{\text {st }}$ Street and Veirs Mill Road as illustrated in Figure 1.4.6 below. Permanent Bluetooth sensors are installed at the end points of this 0.9 mile segment. Travel time was assessed for the period of January $15^{\text {th }}$ to February $1^{\text {st }}$ for 2013 and 2015. The roadway has three lanes in each direction, is signed at $40 \mathrm{MPH}$, and traffic encounters two signalized intersections along the route.

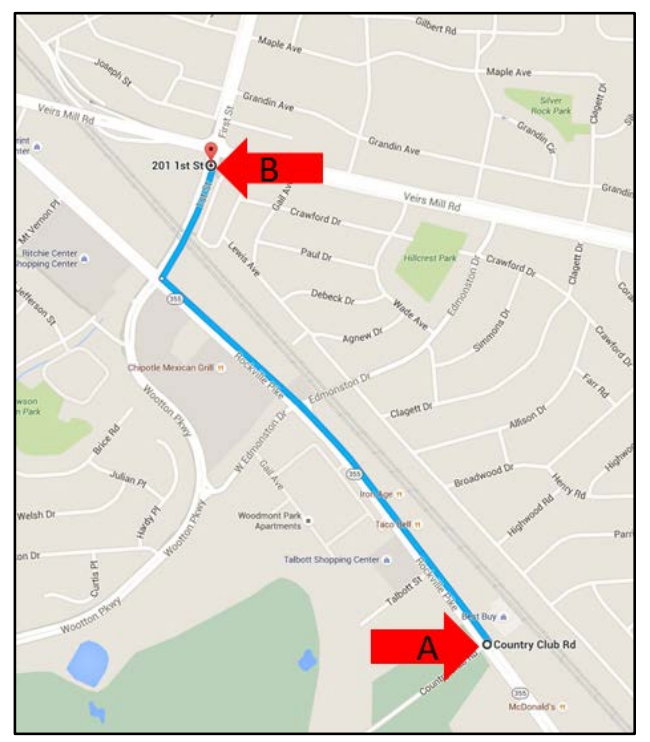

Figure 1.4.6 Sample segment on Maryland Route 355

The travel time CFD for the evening peak period of 5 PM to 6 PM for 2013 (in blue) and 2015 (in red) is shown in Figure 1.4.7. The graph shows the increase in travel time from 2013 to 2015, as the CFD has shifted to the right. On average the travel time on this route increased by over a minute. Reliability of the travel time has changed little, maintaining a similar distribution from 2013 to 2015. 


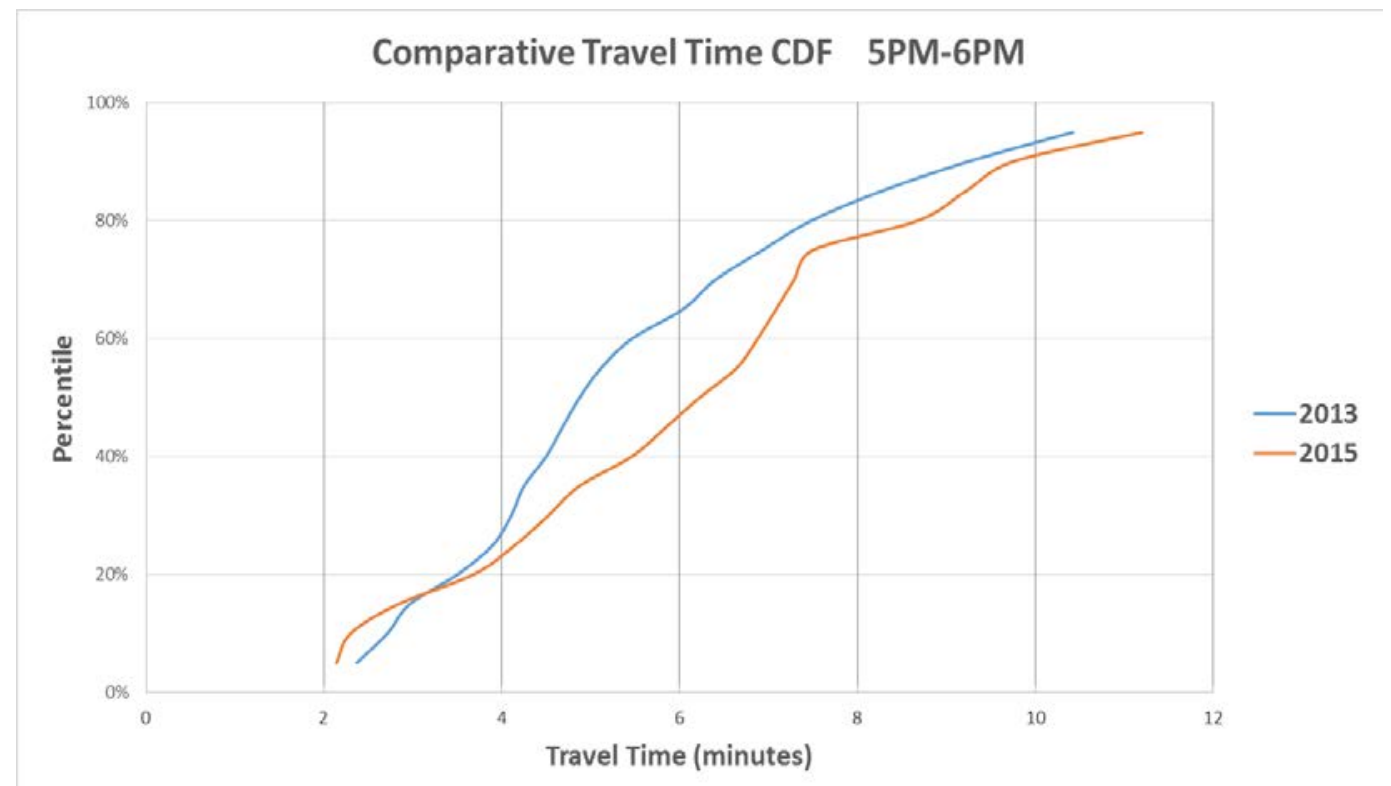

Figure 1.4.7 Comparative CFD of segment travel times from 2013 and 2015

\section{Chapter 2: Quality of Progression with Purdue Coordination Diagrams}

\subsection{Construction of Purdue Coordination Diagrams from High-Resolution Data}

In addition to measured vehicle travel times discussed in Chapter 1, a complementary data source that can provide additional intelligence on traffic operations at a high level of detail is high resolution controller event data. This data consists of timestamped events that occur at signalized intersections, which are recorded to the nearest tenth of a second or better, by equipment in traffic cabinets, in particular by software running on signal controllers. The data consists of a detailed record of each time a signal phase or overlap changes its output state, each time a vehicle detector changes from an active to inactive state or vice versa, and various other events that are significant to controller operation. At the time of writing, most of the signal controller vendors in the US have developed the ability to log this type of data into at least one controller model.

While there are many aspects of signal control that can be analyzed using high-resolution data, this document discusses two particularly important topics that are common to most agencies' operations. This chapter examines the performance of progression, which is the unhindered movement of arriving traffic through signalized intersections with as little stopping as possible by careful scheduling of green indications. The following chapter discusses the quality of capacity allocation, by which the controller divides up time among competing movements.

A fundamental concept common to the discussion of both topics is the cyclic nature of signal control. That is, the state of the outputs regularly transition through red, green, and yellow states, and traffic has a response to this. To make sense of what is occurring at the signal, the intervals are grouped together as illustrated by Figure 2.1.1. The key concept here is the establishment of the effective red and green intervals, which are actually used for movement by vehicles. These differ from the actual output states, due to driver reaction times. The beginning of effective green is generally delayed by the start-up lost 
time, while the end of effective green does not exactly coincide with the start of yellow but follows it by a few seconds. The Highway Capacity Manual suggests using a value of 2 seconds for each. In summary, the key intervals are the effective red and effective green — which vehicle arrivals can be associated with. Naturally, it is preferable to have more vehicles arriving during green, in order that they are more likely to be able to pass the intersection without stopping.

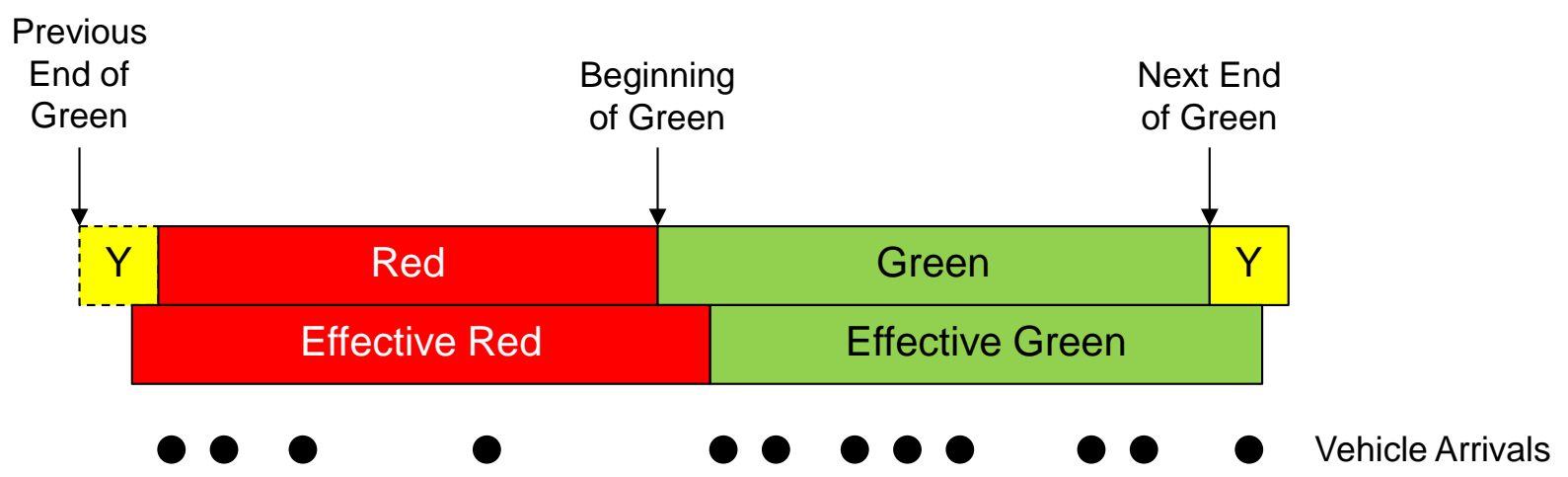

Figure 2.1.1. Concept of a signal cycle.

This chapter will primarily discuss the analysis of patterns in vehicle arrivals using a visualization called the "Purdue Coordination Diagram" (PCD). Figure 2.1.2 explains how this graphical tool relates to traffic performance at an intersection. The figure shows a time space diagram that shows vehicle trajectories as black lines, and the state of a signal located at a position of $3000 \mathrm{ft}$ along the roadway, using red bars to indicate when that signal is red. In this example, suppose that there is a detector located at $2600 \mathrm{ft}$. That detector can be used to measure when a vehicle is approaching the intersection, by projecting its detection time to the likely time when it would arrive at the next intersection. In Figure 2.1.2., this projection is shown by the diagonal dashed lines indicating the end of green (EOG) and beginning of green (BOG) meaning the effective reds and greens. As in Figure 2.1.1., the relevant interval grouping is an effective red followed by the next effective green.

From the trajectory data, it is clear that six vehicles arrive during red and have to stop, while a larger number, arriving in a platoon, arrives during green and is mostly unimpeded. However, in practice, it is very difficult to develop the full picture of the arrival dynamics. Trajectory data has historically been very difficult to obtain, and while technologies are improving, it will likely be years before that data is available for most of the vehicle fleet. Video coverage of the approach can enable qualitative visual analysis, but it is desirable to be able to develop a more complete record of operations for both directions, for all times of day, and for many different intersections. The coordination diagram, shown here as a horizontal bar just above the time-space diagram, is a means of succinctly representing the arrival performance during the cycle. The length of the bar shows the durations of the effective red and green intervals, while the positions of the dots show the times of vehicle arrivals relative to those intervals. This is essentially the basic unit of a PCD: a trace of the relevant arrival events for one particular signal cycle. 


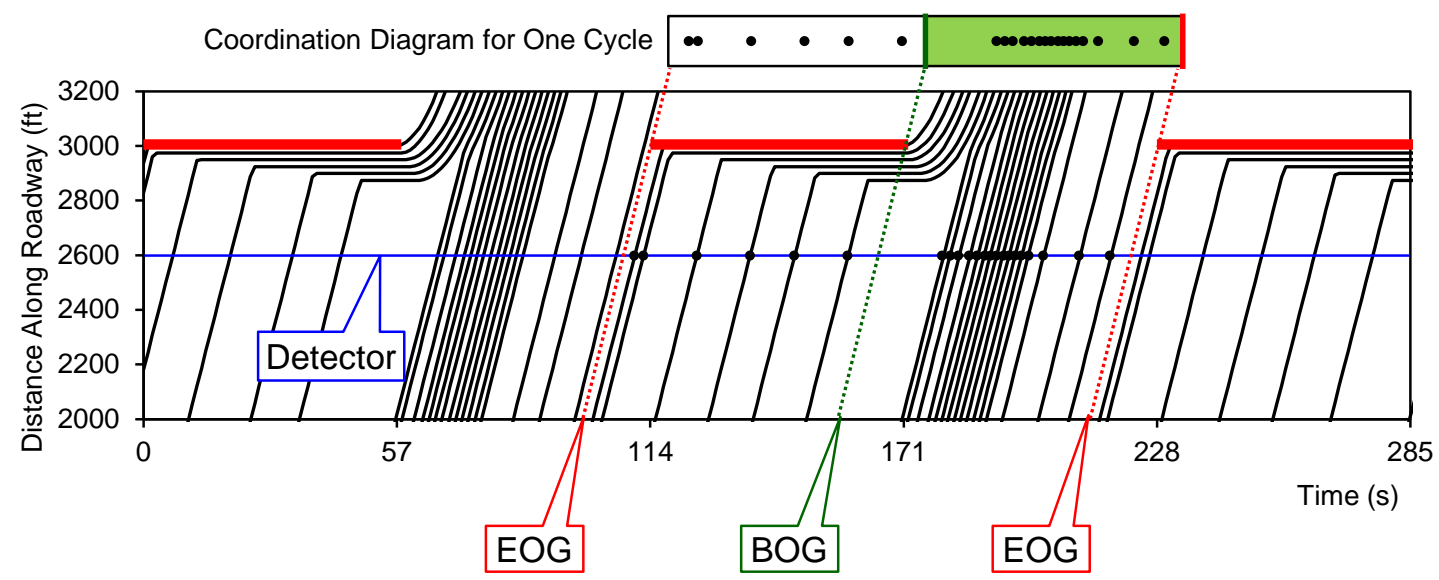

Figure 2.1.2. Overview of the PCD concept.

Figure 2.1.3 presents a more typical use of a PCD, showing several cycles in succession. Here, the individual cycle diagrams have been rotated so that they show the time in cycle along the vertical axis. The horizontal axis shows the time of day. Each column is one cycle, showing first the red interval (along the bottom) and the green interval (at the top). The green portion can optionally be shaded for emphasis. When plotted according to both time in cycle and time of day, the vehicle arrivals (black dots) end up appearing along diagonals across each cycle column.

A number of patterns in traffic can be observed in the PCD:

- A tightly-clustered line of dots (Figure 2.1.3, callout “i”) represents a platoon of vehicles. For many coordinated arterials, there will often be one large platoon that represents traffic coming from the through movement at the upstream intersection. However, it is also possible to see other platoons (Figure 2.1.3, callout "ii”) that are likely to consist of vehicles that originated from upstream turning movements.

- There is substantial variation in the amount of green time, due to actuation. For the first cycle, the beginning of green occurs around 50 seconds into the cycle, while the fifth cycle (around 17:10) has the start of green around 15 seconds. Early return to green can thus be directly observed in the PCD.

- The beginning of red line also indicates the cycle length. In Figure 2.1.3, the duration of each cycle is uniform across the observed data. This makes a very clean example graphic, but will not always be the case, as later examples will demonstrate. There can sometimes be very substantial variation in the cycle length, even within a short time period. 


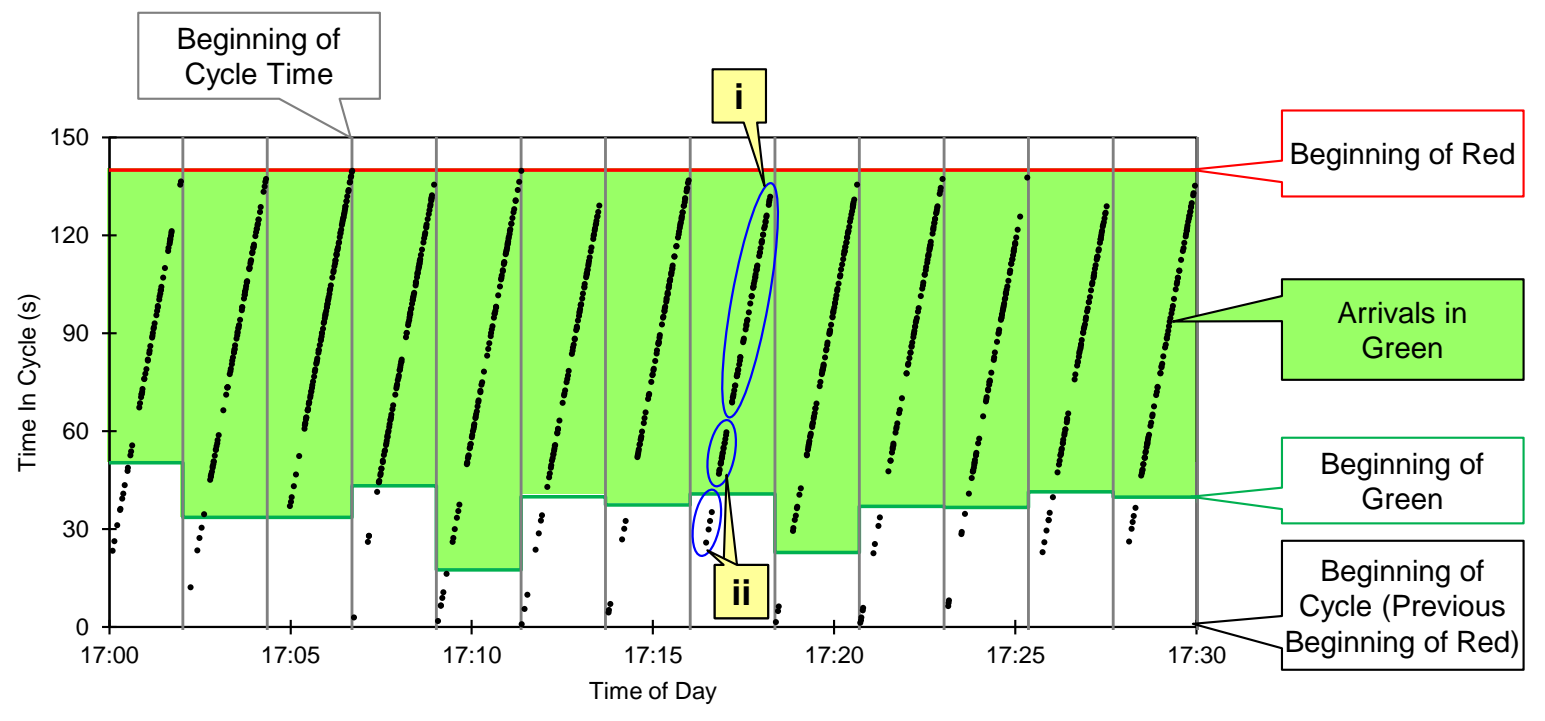

Figure 2.1.3. A PCD for a 30-minute period.

Figure 2.1.4 expands the view from a half hour to a 24-hour period. The lower plot shows a PCD while the upper plot shows the percent on green (POG) for each cycle (dots), with a 20-cycle moving average (line). Each graphic is divided into regions by the vertical blue lines, which show when pattern changes occur. This particular intersection runs free (fully-actuated, non-coordinated) from 0:00 to 5:00, and then rotates through patterns $1,2,3,4$, and back to 1 , switching at 5:00, 11:00, 13:30, 15:00, and 19:00. The cycle length remains the same throughout all of the pattern changes, but the splits and offsets are adjusted—as can be seen by differences in the arrival patterns from one pattern to the next.

The POG plot shows that the quality of progression tends to be exceptionally good at this approach throughout most of the day. The average POG hovers around $90 \%$ for almost the entire day. Out of the four time-of-day patterns, pattern 3 seems to have slightly lower POG than the others, although the value is still quite high.

The PCD offers several observations that can be made for each pattern:

- During the early-morning free period, there are very sparse arrivals, and cycle lengths tend to become very long as the signal dwells in green on the mainline, and very little demand shows up on conflicting movements. Toward the end of the period, the signal begins cycling a little more actively.

- After 5:00, pattern 1 goes into effect, holding the cycle length at around 136 seconds for most of the pattern. There is some variation at times due to actuation. Early in the pattern, the platoons are quite small, but these grow to fill most of the green time by the end of the pattern. The arrival pattern appears to have two distinct platoons, with one cluster of arrivals that occurs after about 30 seconds while the second arrives after about 60 seconds into the cycle. 
- After 11:00, pattern 2 is in effect. The overall arrival characteristics are about the same, but the pattern is shifted slightly later, due to a different offset. Both of the two platoons arrive inside of the green interval.

- After 13:30, the controller implements pattern 3. The overall shape of the point cloud of arrivals is nearly the same as before, but appears to be shifted downward a bit (earlier in cycle). The first platoon arrives somewhat before the start of green, which would explain why POG is slightly lower for this pattern.

- The PM peak begins at 15:00 when pattern 4 is used. Again, there is some shifting of the arrivals due to offset changes, but overall the progression appears to be very good with most of the dots coincident with the green. The amount of traffic becomes greatest during this time period, as shown by the filling of the space with an increasing number of dots, especially around 17:00 or so. The two platoons come close to merging together around that time.

- In the evening, pattern 1 goes back into effect and its characteristics are similar to the morning again. As the end of the day arrives, the platoons become smaller and smaller and vehicle arrivals become more and more sparse.

In summary, the PCD can be easily read using the knowledge that dots above the green line are "good" and dots below the green line are "bad". However, there are many other traffic conditions that have a particular signature in the PCD, as the next section will discuss. 

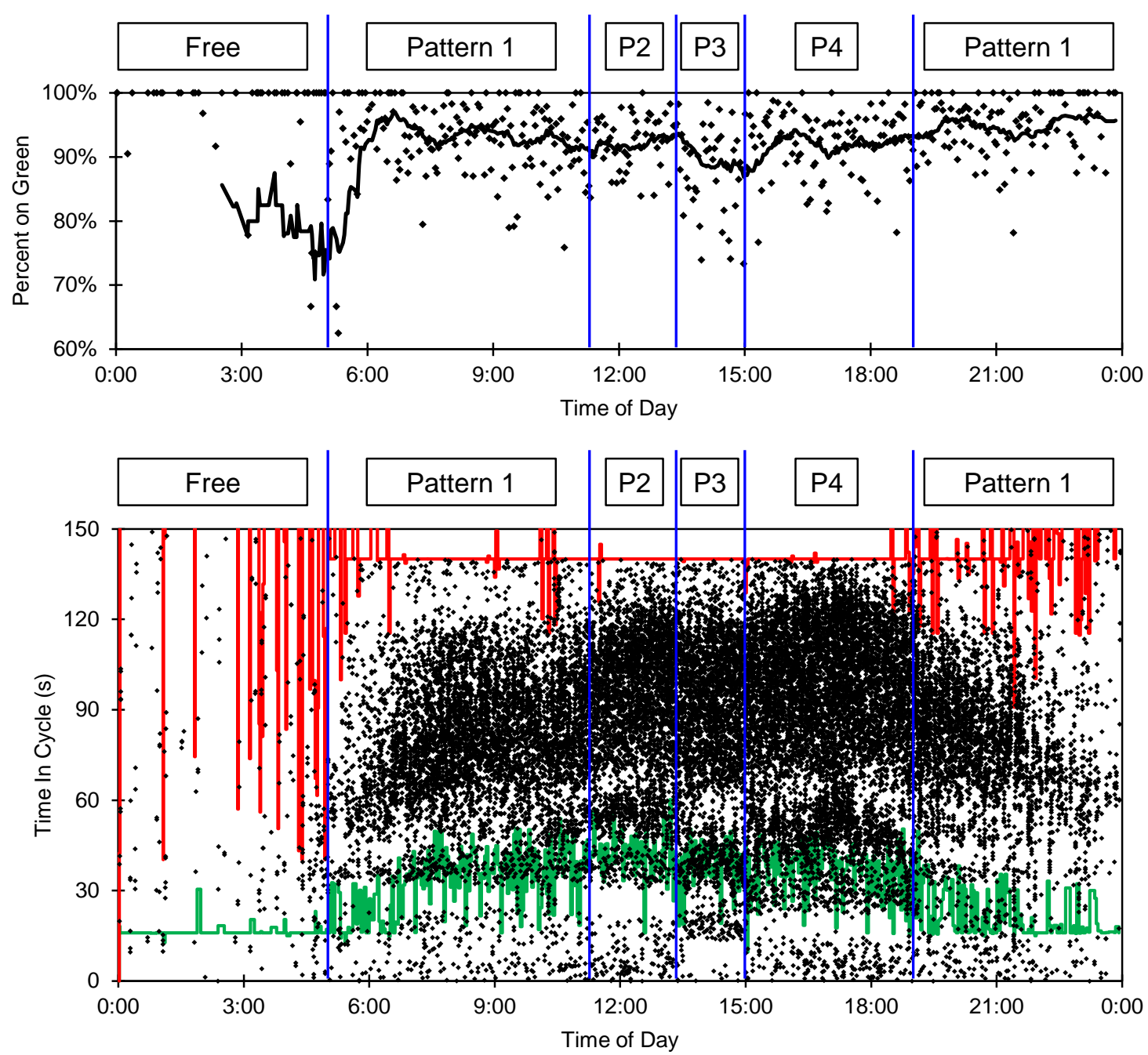

Figure 2.1.4. A 24-hour PCD compared with the percent on green metric. 


\subsection{Signal Control Attributes Observable in PCDs}

This section presents examples of a variety of traffic conditions and the types of features that they exhibit in a PCD. The examples are drawn from many different locations in Indiana, and in each case the graphics show the arrival times of vehicles, as determined by a detector located about 5 seconds upstream of the intersection.

Figure 2.2.1 shows an example of what could be considered exceptionally good progression. This PCD represents operations at the exiting approach of a diamond interchange. During the AM peak (5:00-9:00) and PM peak (14:00-19:00), virtually all of the vehicle arrivals occur within the green. Such favorable progression is possible when there is a portion of arrival-free time to line up with the red interval. In this example, the AM and PM peaks exhibit a large portion of the cycle where no arrivals occur; interestingly, the rest of the day shows a rather different pattern, which may result from a different phase sequence being used at that time. Additionally, the AM peak exhibits a substantial amount of time in which the signal is green but almost no traffic is being served in this direction. It is likely that traffic in the opposite direction is being served during this part of the cycle, but in general it is preferable (although sometimes unavoidable) to avoid periods of "wasted" green time.

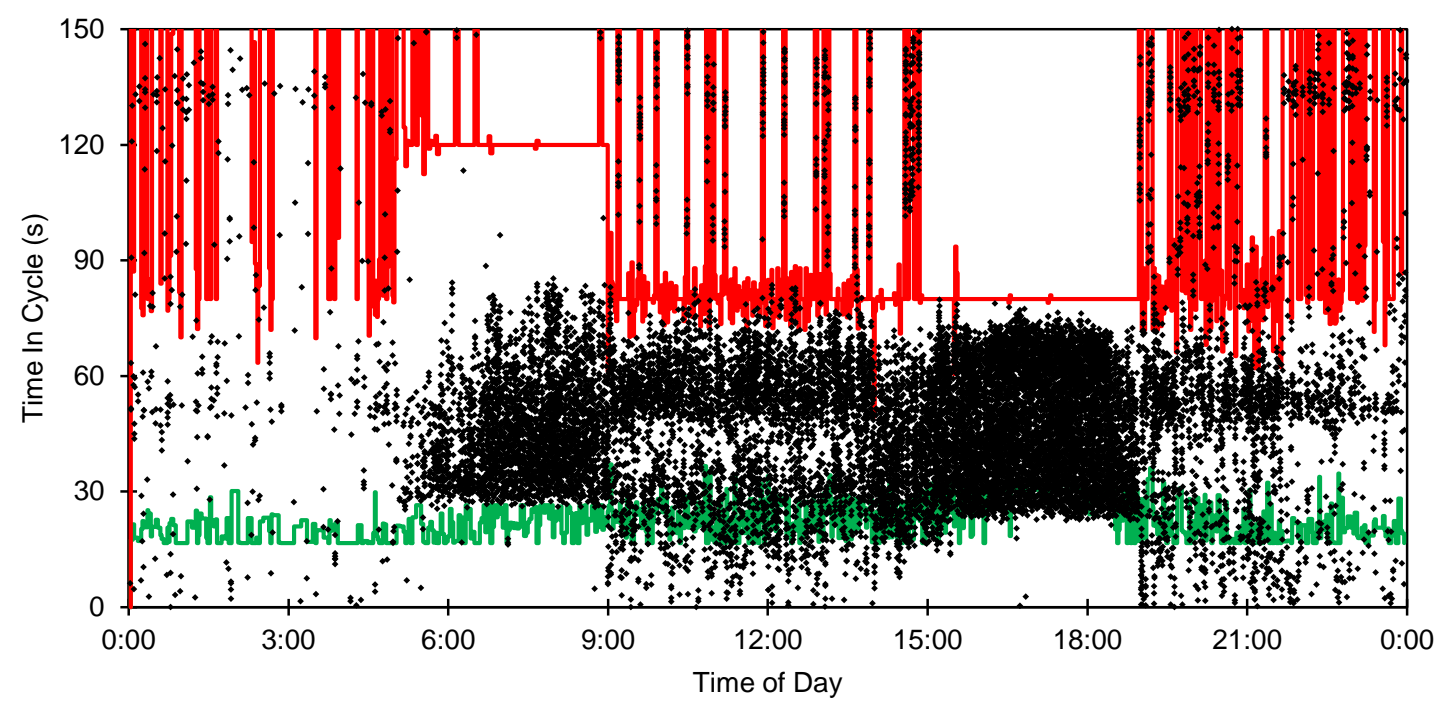

Figure 2.2.1. Example of good progression. 
Figure 2.2.2 shows a PCD for an approach where there is no upstream intersection. At such a location, the expectation is that the arrivals will be completely random, and the PCD exhibits no evidence of repeating platoons. There is some growth of traffic during different parts of the day, with the AM peak having a visibly darker point cloud than the rest of the day. This is typical for the first intersection along a corridor, where the closest nearby intersection is more than a few miles away, or if the neighboring intersection operates in a fully-actuated, non-coordinated mode. Queue formation and dispersal at the first intersection creates the platoons that have the potential to progress through the other intersections.

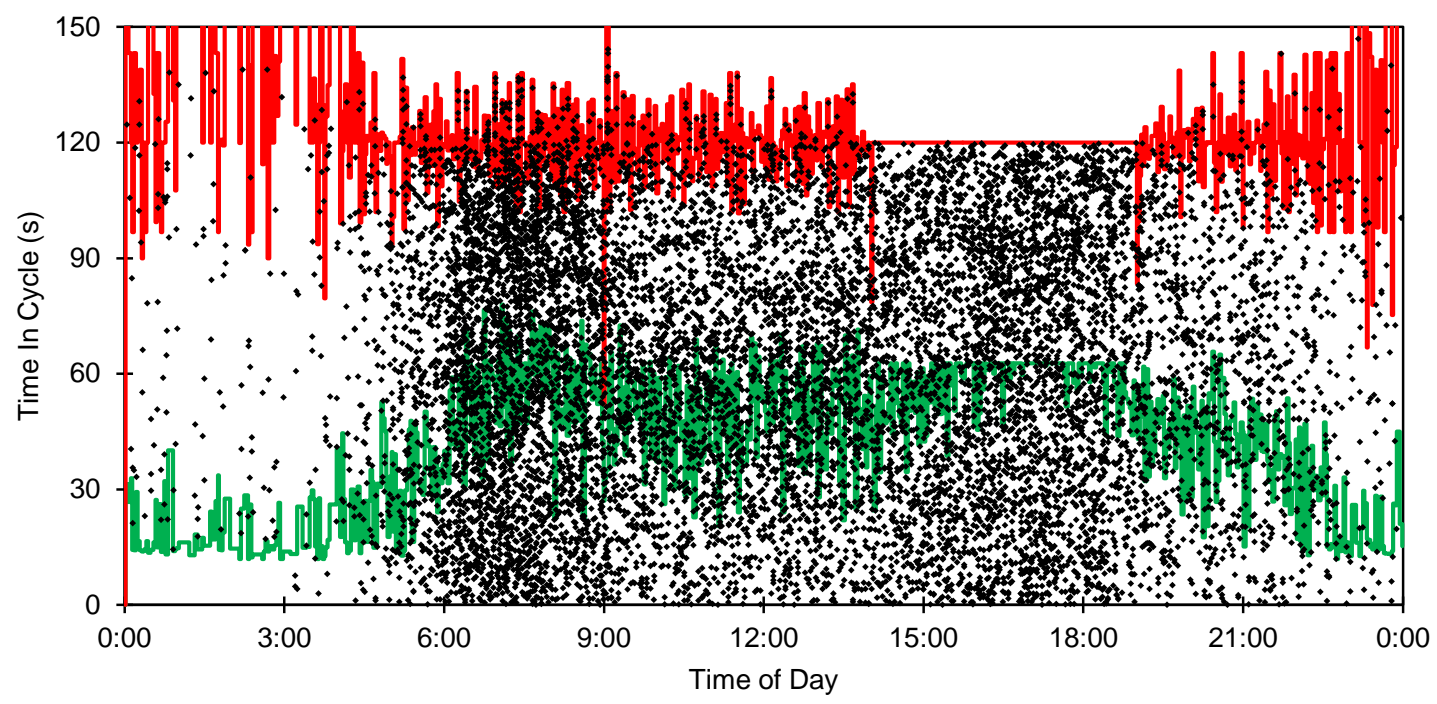

Figure 2.2.2. Example of randomly arriving vehicles. 
Figure 2.2.3 shows an example of poor progression. The arrivals during the PM peak (15:00-19:00) are heavier in the red than in the green, in this example. There are still some arrivals during green, but the heaviest portion of the arrivals fall below the green line. The midday portion from roughly 9:00 to 15:00 shows arrivals distributed throughout the cycle and there is not a strong pattern of platoons. Rather, the PCD shows that there are some parts of the cycle where there are fewer arrivals, but this occurs during the later portion of green rather than the red, which would be preferred. By contrast, the AM peak in particular (6:00-9:00) exhibits relatively good progression (arrivals in green) with well-formed platoons. This intersection occurs along a corridor where one direction or the other is favored at different times of day. During the AM peak, the direction of the PCD is favored, while at other times of day the opposing movement is favored.

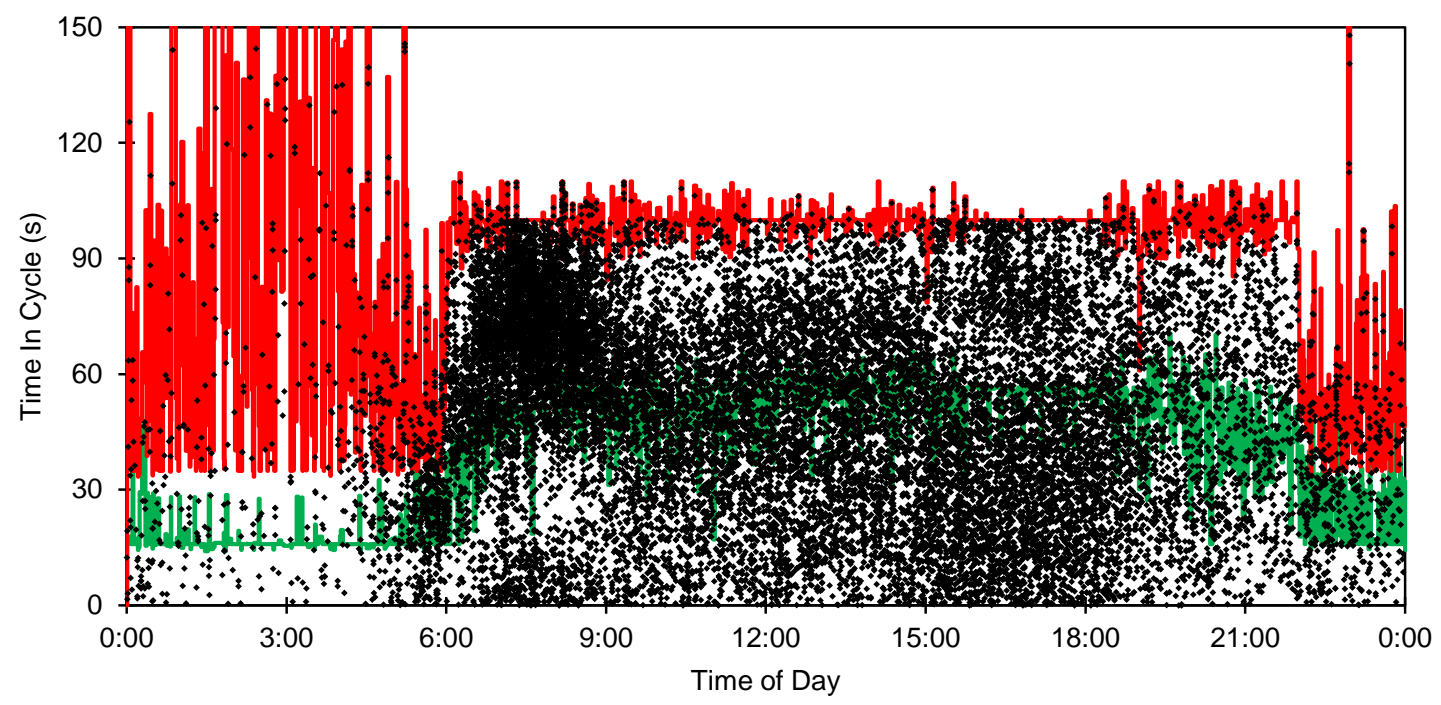

Figure 2.2.3. Example of poor progression. 
Intersections under free (fully-actuated, non-coordinated) operations can still produce PCDs and these can still provide useful information. Figure 2.2.4 shows a PCD for a location where the signal runs free for most of the day, with the exception of a 15:00-19:00 coordinated time period. Under free operation, the effective cycle length expands and contracts according to the demand. During the overnight period, there is very little side street demand, and the signal dwells in green on the mainline for long periods of time. However, after 05:00, the intersection begins cycling regularly and continues to do so throughout most of the day. Cycle lengths become as high as 120 seconds or so near 07:00 (callout "i”) and then shrinks back down during the midday. The arrivals appear to be random throughout this time because the neighboring intersection is also not coordinated. However, during the PM peak (15:00-19:00), the cycle length is fixed at 116 seconds (callout "ii”) and there is a regular pattern of platoons during most of the cycle. This pattern disappears after roughly 18:00, which suggests the possibility that the upstream intersection might drop coordination earlier. The remaining fully-actuated portion after 19:00 bears similarities to the midday.

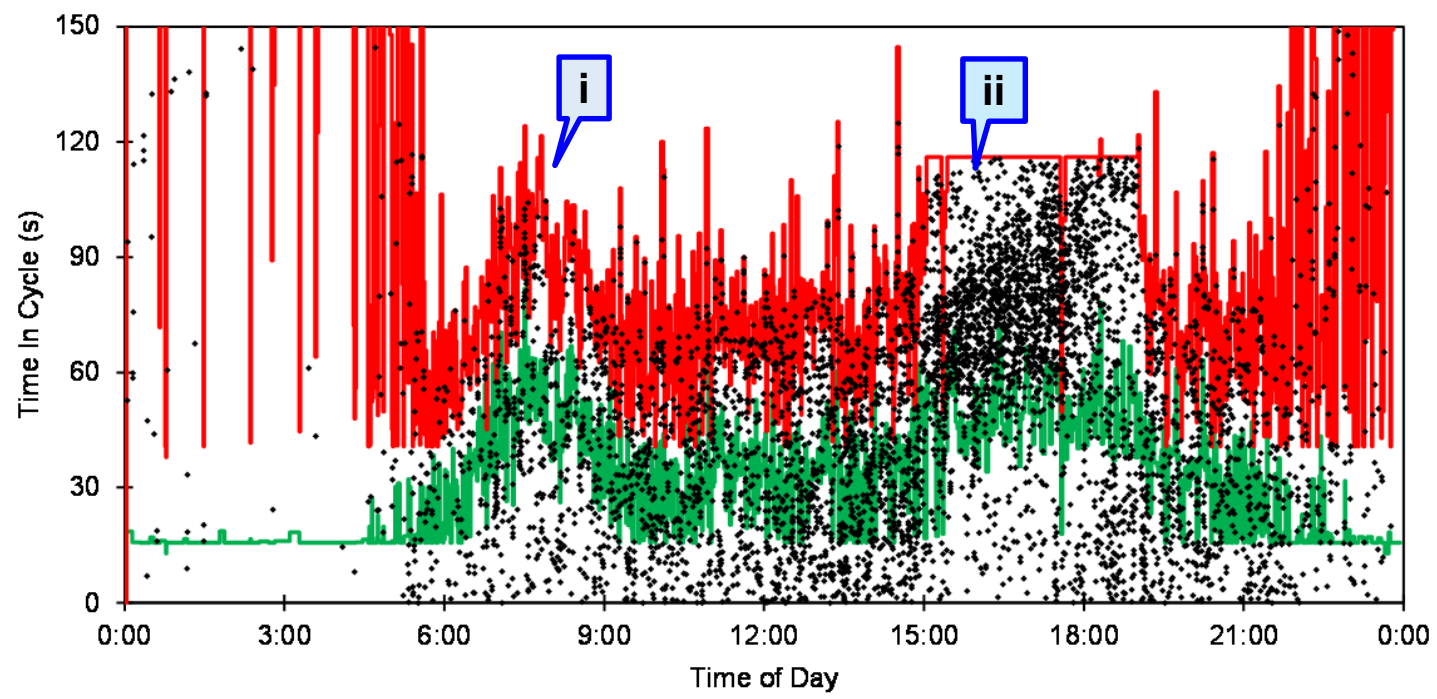

Figure 2.2.4. Example of fully-actuated operation. 
Saturation of a signalized movement occurs when the demand approaches or exceeds the available capacity. In general, long queues form at the intersection and these may not clear during every cycle. Consequently, some vehicles will require multiple cycles to pass the intersection. PCDs depend on the use of a detector in advance of the intersection to measure incoming arrivals. When the movement is saturated, queues will begin to build and these may extend as far back as the detector. When this occurs, a particular pattern often appears in the PCD due to the fact that traffic is stopped over the detector for a certain time and that traffic is moving for the rest of it.

Figure 2.2.5 shows an example of an approach that accepts traffic coming directly from a freeway offramp. During most of the day, the arrivals appear to be completely random, which is what would be expected. However, after 15:00, the pattern changes considerably. A gap where very few vehicle detections occur (callout “i”) is visible during a part of the cycle. This represents where vehicles are queued over the advance detector. A very dense cluster of dots can be observed above the gap, which is approximately 30 seconds after the start of green. This represents traffic moving at the saturation flow rate across the detector; the gap represents the time it takes for the departing queue to reach back as far as the advance detector. After the end of green, traffic begins queueing again, which tends to be spread out over the red interval as drivers are less likely to hurry toward a red light as to a green one. A pattern such as this in the PCD is a sign of saturated conditions.

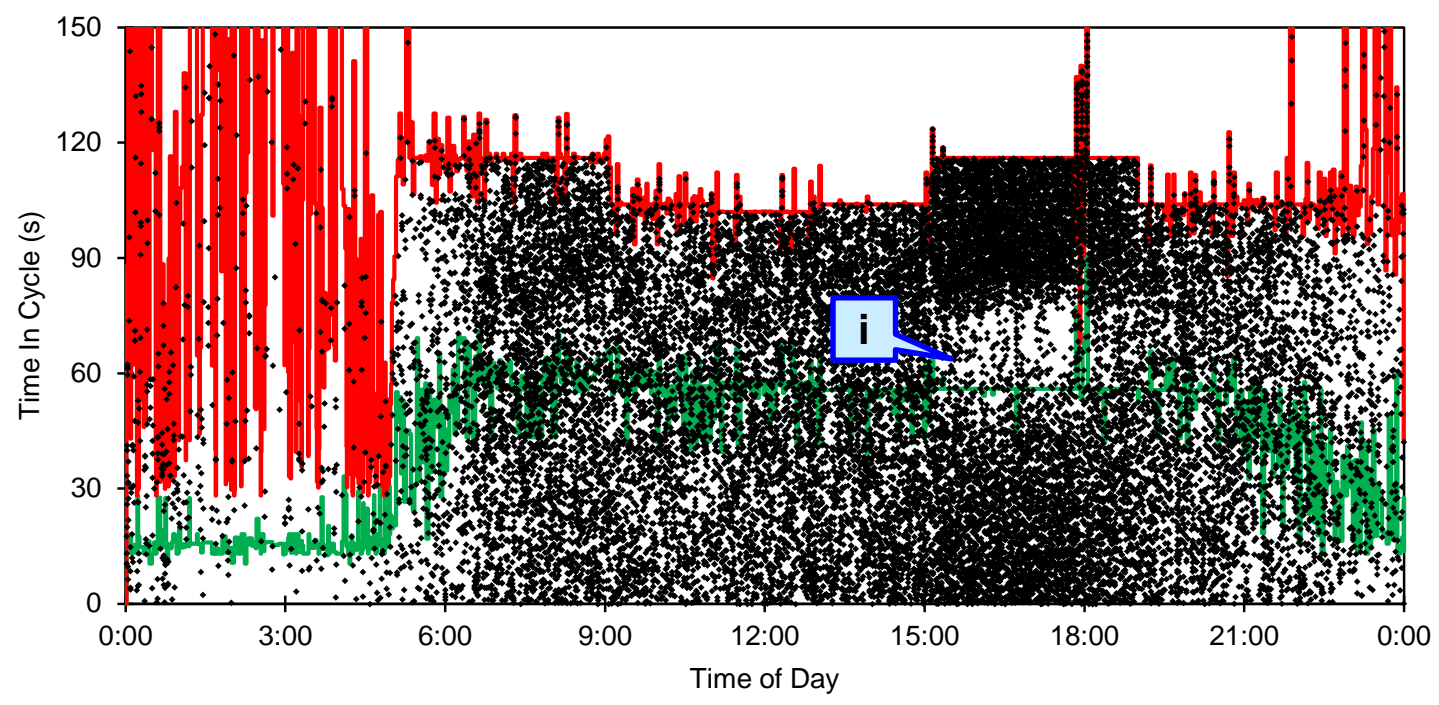

Figure 2.2.5. Example of saturated operations during the PM peak. 
Figure 2.2.6 shows a PCD with an interesting arrival pattern that features diagonal striping during certain TOD plans. Figure 2.2.6a shows the 24-hour view while Figure 2.2.6b focuses on the 9:00-11:00 time period. The striping occurs because, during each successive cycle, the platoons are arriving at a slightly earlier time in the cycle. For about half of the time, the platoons arrive during green, and the other half of the time they arrive in red. This pattern happens when the cycle length at the neighboring intersection is set to a different value from the local intersection. In this particular example, the upstream intersection had a controller error which caused technicians to swap out the controller with a spare unit. However, the new controller did not have the most up-to-date timings. Hence, during certain times of day the cycle lengths were in agreement (6:00-9:00, 11:00-13:00, 15:00-19:00) while at others the cycle lengths were different (9:00-11:00. 13:00-15:00, 19:00-22:00).

The previous chapter showed examples where a similar pattern appeared in the travel time data during a cycle length mismatch (Figures 1.3.3, 1.3.4, and 1.3.5). Figure 2.2.6 illustrates why such a pattern might occur in the travel time data. The PCD shows that the platoon arrives earlier in each successive cycle. There are some cycles where the traffic is progressed serendipitously, simply because the upstream and downstream greens happen to agree for that particular moment. However, a few cycles later, the arrivals have begun to wind to an earlier part in the cycle where it is red; they then become earlier and earlier in the red, meaning that with each successive cycle, the platoons must wait increasingly longer after stopping, which means that delay increases.

The travel times in Chapter 1 show patterns where the travel time rises to a high value and then gradually decreases. This likely results from a cycle length mismatch where the platoons are arriving slightly later in each cycle. Thus, initially the arrivals occur at the beginning of red, corresponding to the highest travel times, and as the platoons arrive closer to the green, the travel times gradually decrease. 


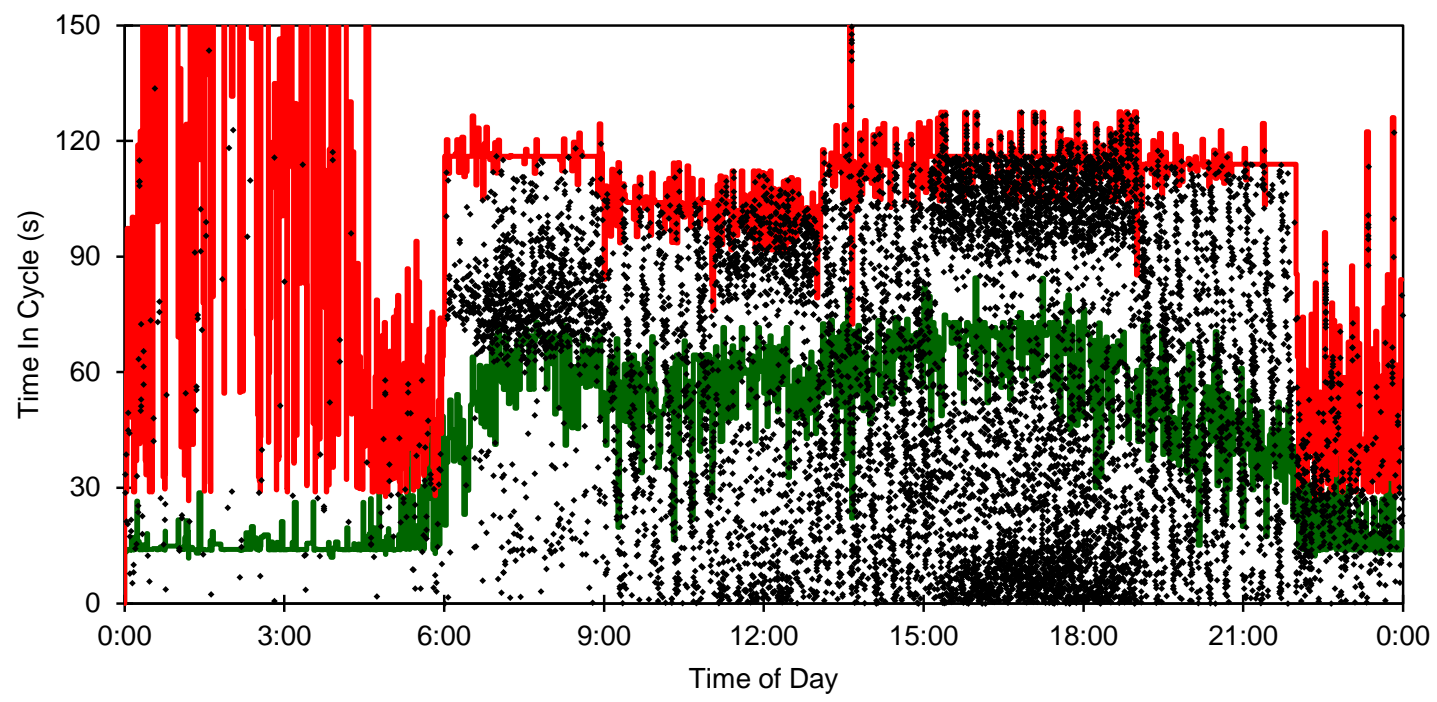

(a) 24-hour view.

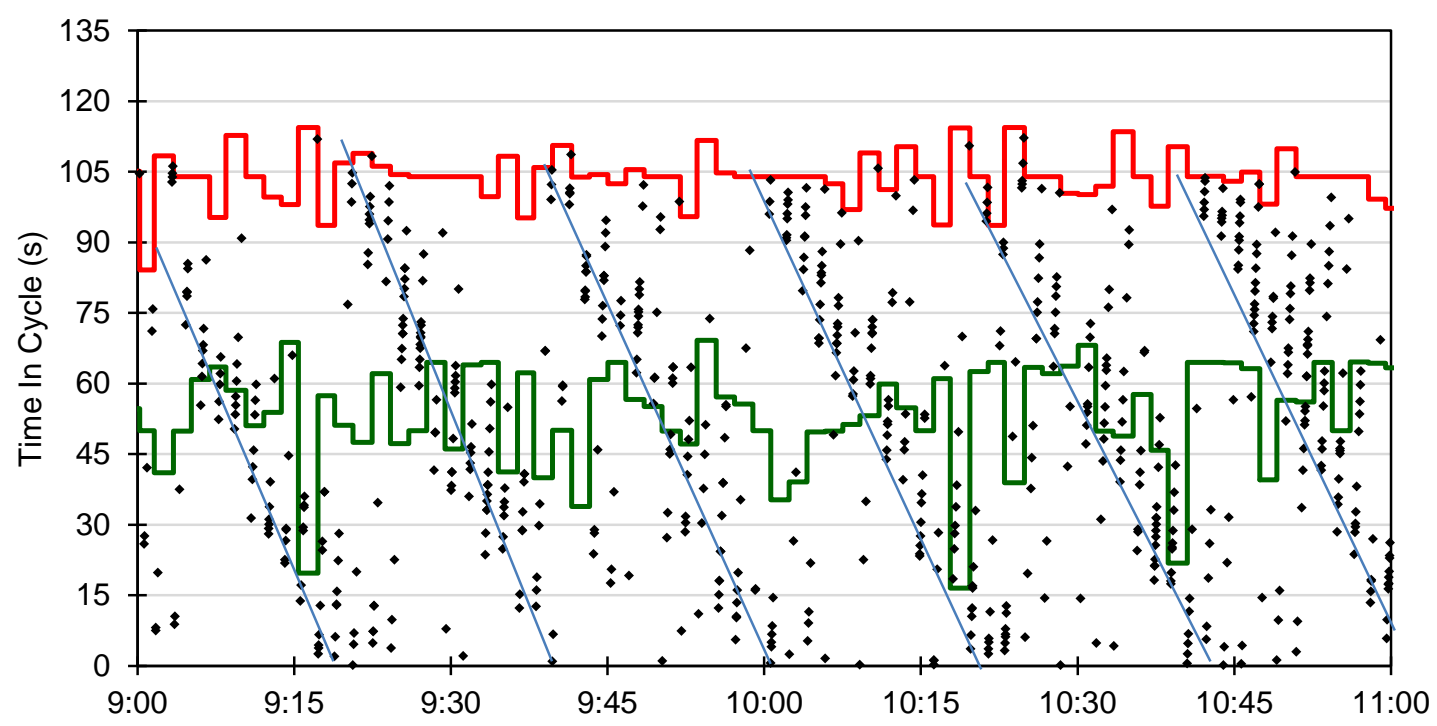

(b) Zoomed into the 09:00-11:00 period.

Figure 2.2.6. Example of incorrect timing plan at the upstream intersection, showing some times of day with mismatched cycle lengths. 


\subsection{Before/After Comparisons using PCDs}

PCDs also provide a useful tool for making comparisons of arterial signal operations under different conditions, such as to determine whether a retiming activity was successful, whether there was an impact from a special event, and so forth. For example, in the previous section, Figure 2.2.6 showed what happened when an upstream intersection had the wrong timing plans in place. Figure 2.3.1 shows the "correct" operation under the intended timing plans. There is no longer any striping due to cycle length mismatches, and progression during the PM peak (15:00-19:00) is improved. However, interestingly, progression during the AM peak (6:00-9:00) is not quite as good as conditions with the incorrect plan in operation.

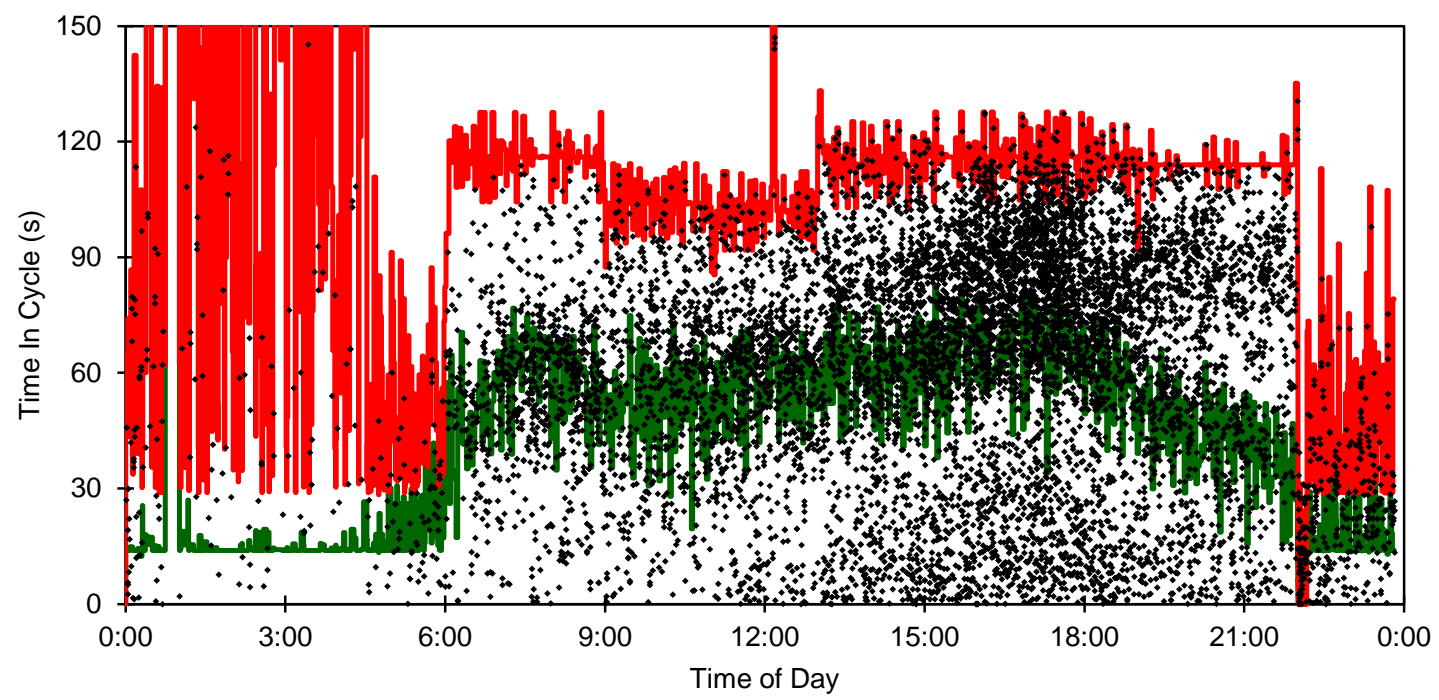

Figure 2.3.1. View of the same approach as shown in Figure 2.2.6, but with the correct timing plans in effect.

PCDs have a great deal of value when considered on a corridor basis. Figure 2.3.2 shows a map of SR 37, which is a four-lane divided highway carrying an AADT of roughly 35,000 vehicles per day. There are eight signalized intersections spanning 5 miles from end to end. The map clusters the intersections into groups of four on the north end and the south end. This arterial serves daily commuter traffic coming to and from Indianapolis (which lies to the south). There is also a substantial amount of retail development along the corridor that generates traffic. This case study will present examples from offset optimization studies that were carried out in 2009 and 2010. 


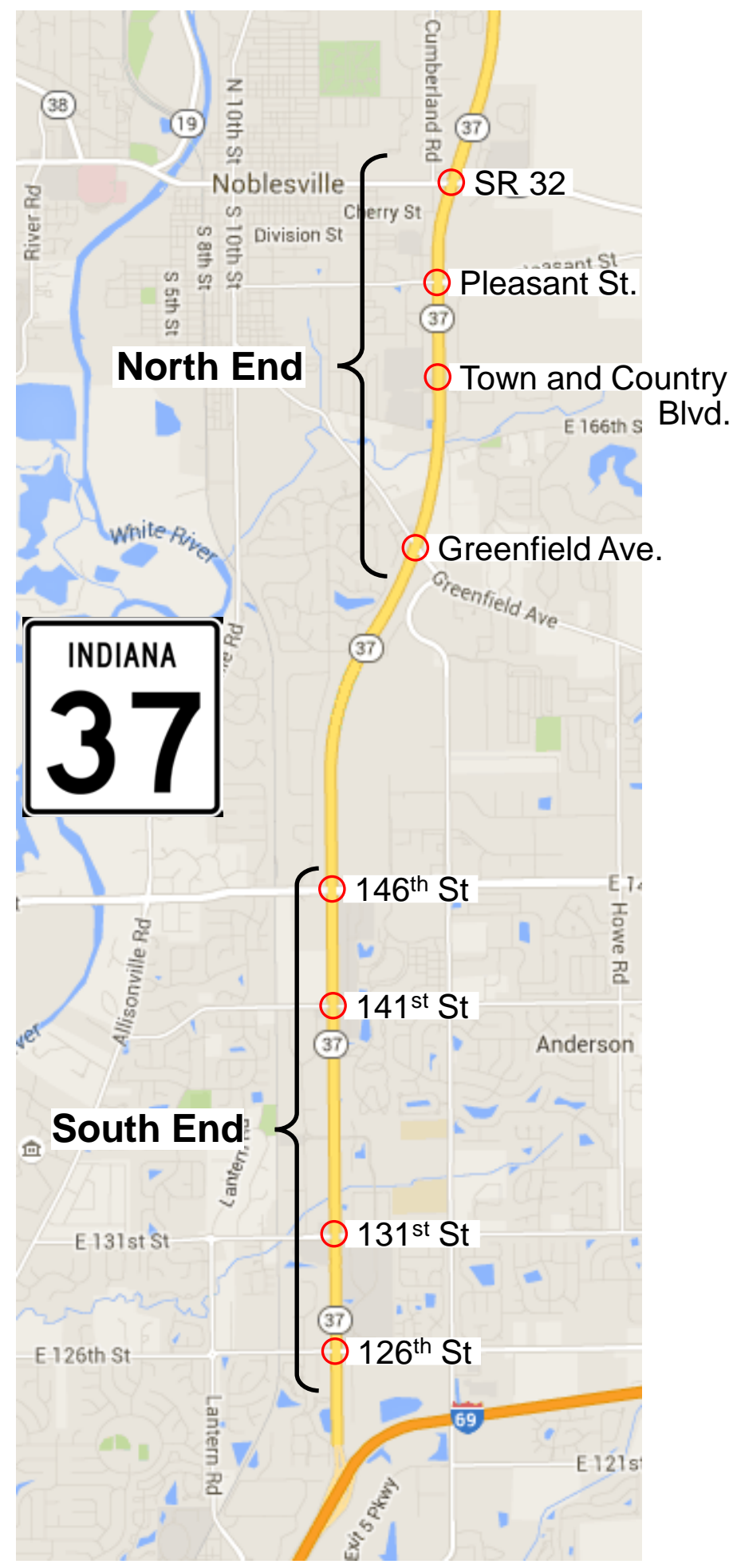

Figure 2.3.2. Example arterial signal system: Indiana SR 37 in the Noblesville area. 
In the summer of 2009, the north end of the system was equipped with controllers capable of logging high-resolution data, and that data was used to support a study for retiming the offsets on the fourintersection corridor. Because SR 37 is one of the busiest commuter arterial routes, its weekday offsets were relatively well-timed, and the quality of progression was therefore relatively good. However, typical of many corridors, the Saturday plan had never been carefully examined during its operation. The timing plan was the best guess of the traffic engineer working with traditional signal timing software. Thus, it was not entirely surprising when the initial results showed an opportunity for improvement.

Figure 2.3.3 shows PCDs for the four intersections (eight approaches, four southbound and four northbound) on the north end of the system, reflecting existing conditions before any of the offsets were changed. Examining the southbound PCDs, the first intersection at SR 32 (Figure 2.3.3a) has random arrivals; the second intersection at Pleasant St. (Figure 2.3.3c) appears to have the densest portion of the vehicle arrivals occurring right at the start of green, so its performance is not very good; while the last two intersections (Figure 2.3.3e and 2.3.3g) both have platoons arriving in green. Looking at the northbound, the first intersection at Greenfield (Figure 2.3.3h) has most of its largest platoon of vehicles arriving in red; the second intersection at Town and Country (Fig 2.3.3f) has its arrivals in green; the third intersection at Pleasant (Fig. 2.3.3d) again has most of its arrivals in red; while finally the last intersection moving northbound (Fig. 2.3.3b) has arrivals in green. In summary, a typical vehicle among arterial traffic along SR 37 would probably have stopped twice going southbound (at SR 32 and Pleasant) and would have stopped twice going northbound (at Greenfield and Pleasant). While odds of stopping at the entry of the corridor is unlikely to change, there are plenty of other opportunities for improvement within this corridor.

Figure 2.3.4 shows PCDs after adjustments to the offsets were made. Comparing these with the "before" PCDs in Figure 2.3.3., several improvements can be seen. The northbound approaches at Pleasant and Greenfield (Figure 2.3.4d and h) now both exhibit arrival in green. The southbound approach at Pleasant (Figure 2.3.4c) now also has somewhat more favorable characteristics. Southbound at Greenfield (Figure 2.3.4g) has shifted and now seems to have somewhat better progression, in that the cluster of dots that most likely represents platoons of through vehicles is now lined up well with the green. Northbound at SR 32 (Figure 2.3.4b) shows slightly less favorable progression, with the main group of arrivals occurring just slightly before the green. Southbound at Town and Country (Figure 2.3.4e) now has arrivals occurring slightly later in the cycle, and the platoons appear to be cut off somewhat as a result. However, there are often unavoidable directional tradeoffs in adjusting offsets.

Another way of viewing the same information more summarily is to consider the change in the percent on green (POG). Figure 2.3.5 shows a map view with pie charts associated with each signalized approach. The pie charts represent changes in POG. Red slices indicate where the POG decreased, while dark green indicate where POG increased. The light green represents the base amount of POG common to both the before and after values. As before, we see two large dark green slices in the northbound direction, while the southbound has one modest red and two dark greens, which correspond to the visual assessment made of Figure 2.3.4. 
(a) SB, SR 32

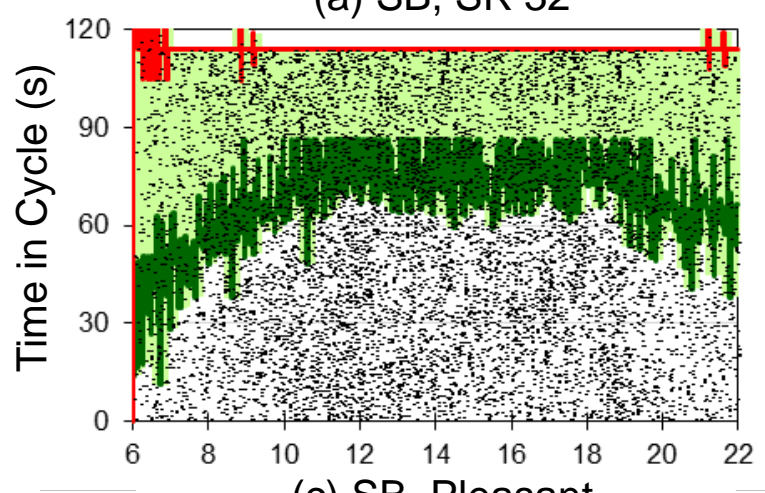

(c) SB, Pleasant

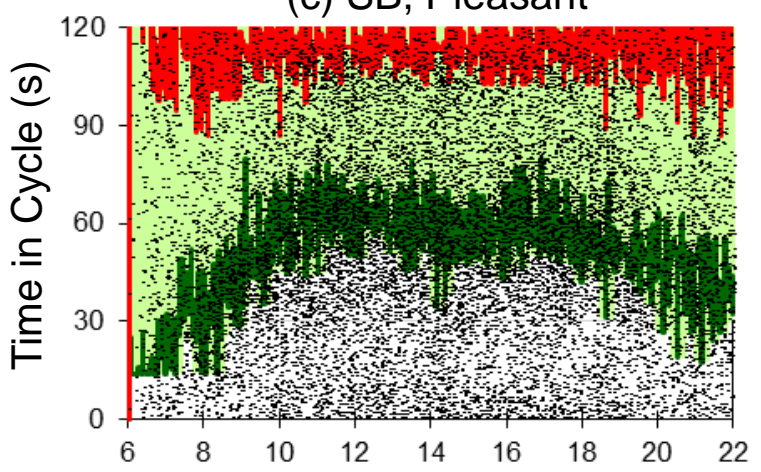

(e) SB, Town \& Country

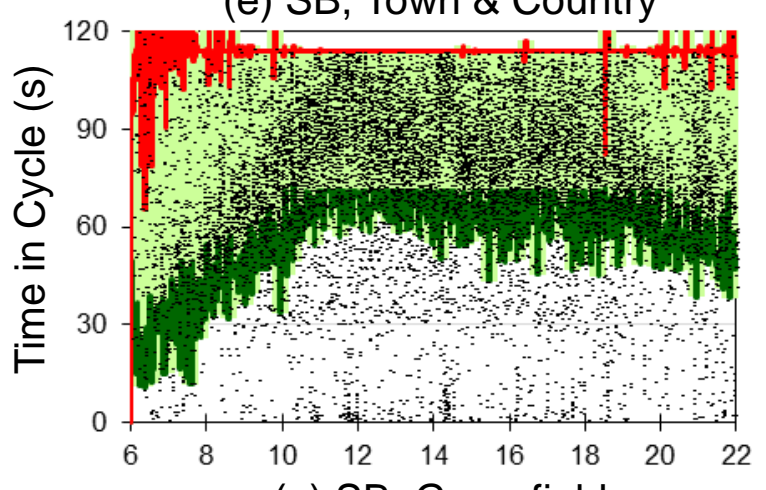

(g) SB, Greenfield

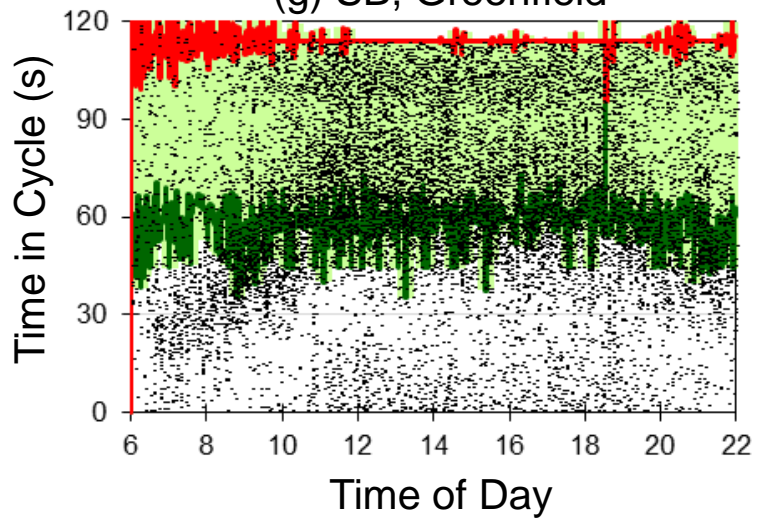

(b) NB, SR 32

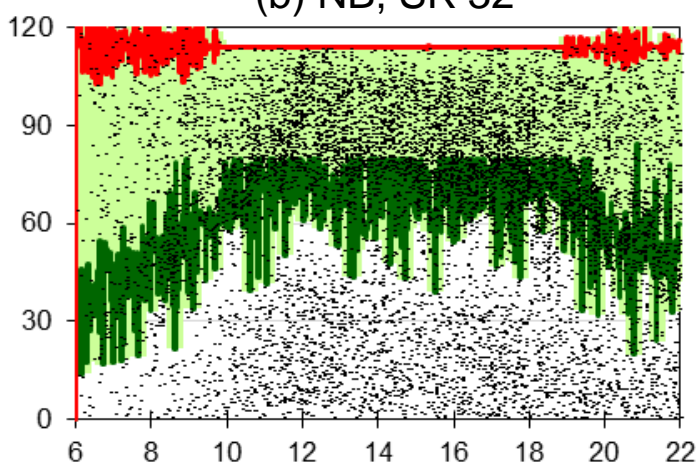

(d) NB, Pleasant

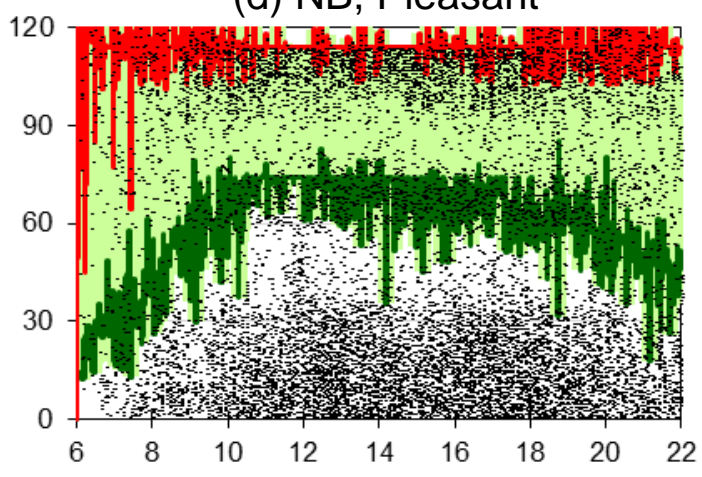

(f) NB, Town \& Country

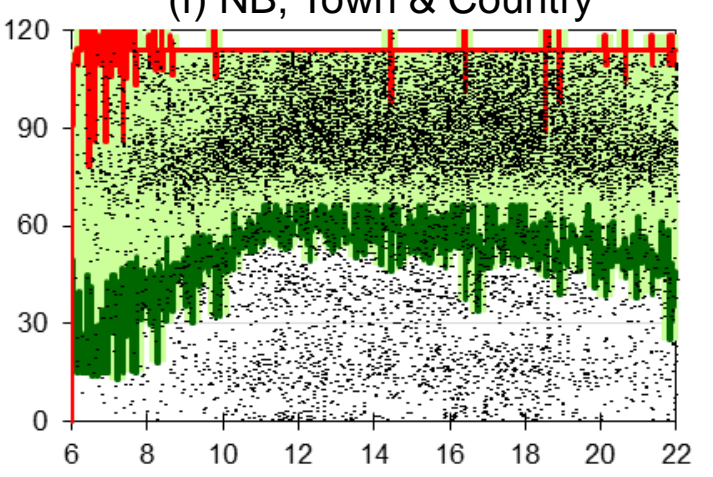

(h) NB, Greenfield

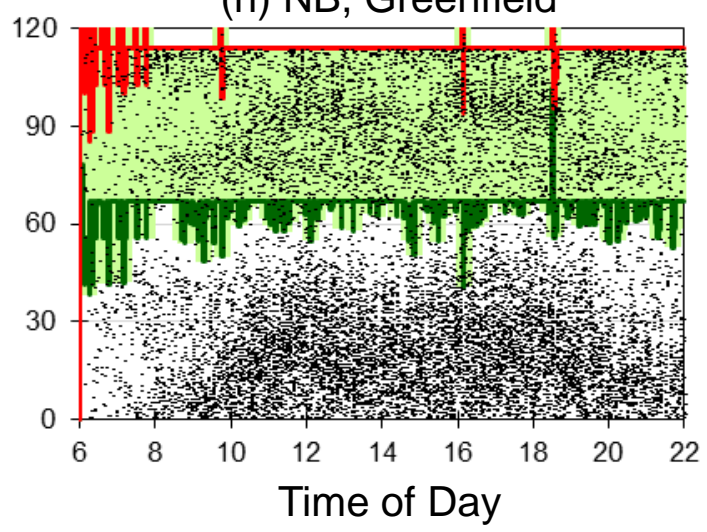

Figure 2.3.3. North End PCDs before 2009 retiming. 
(a) SB, SR 32

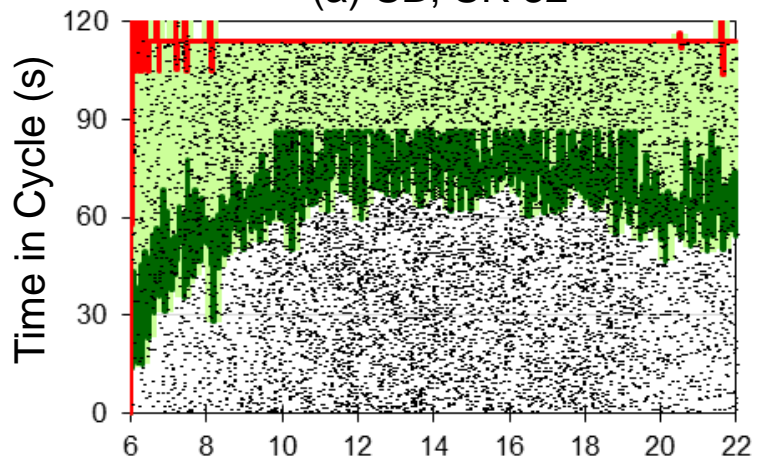

(c) SB, Pleasant

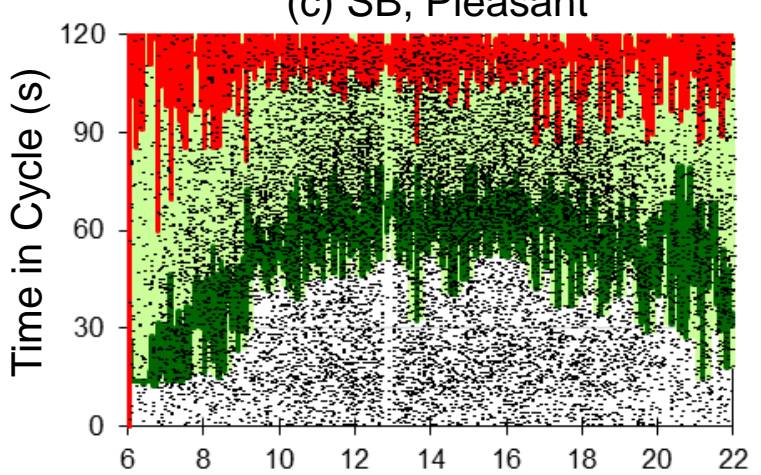

(e) SB, Town \& Country

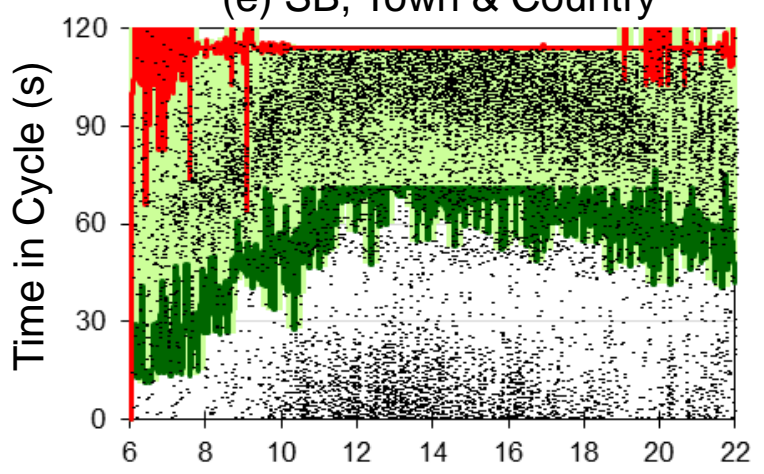

(g) SB, Greenfield

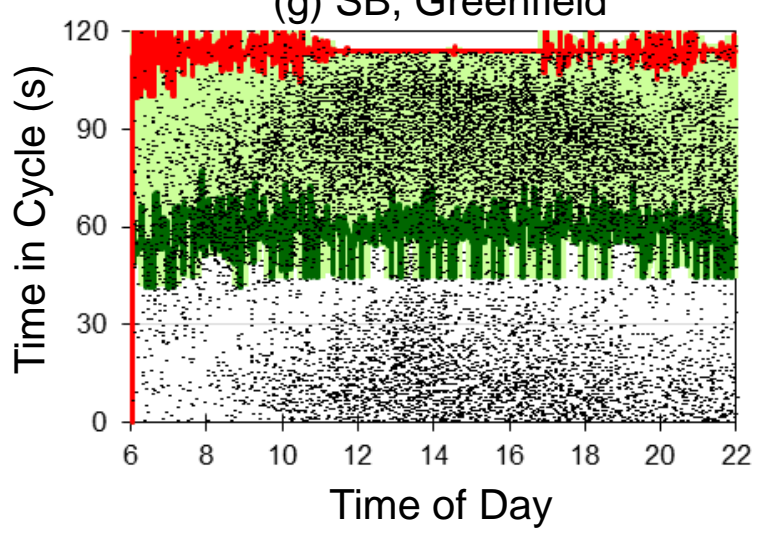

(b) NB, SR 32

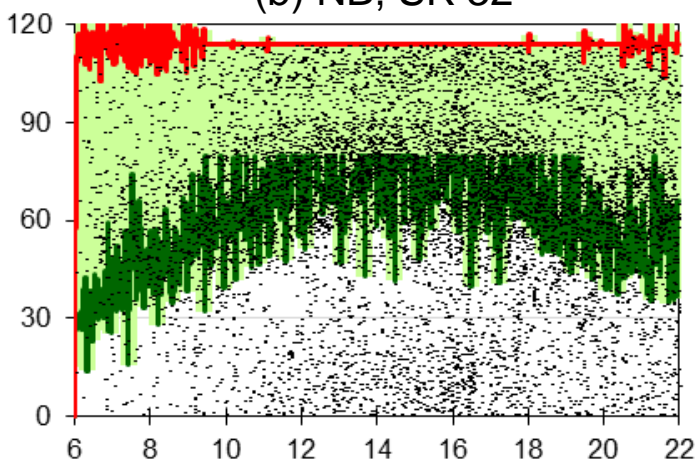

(d) NB, Pleasant

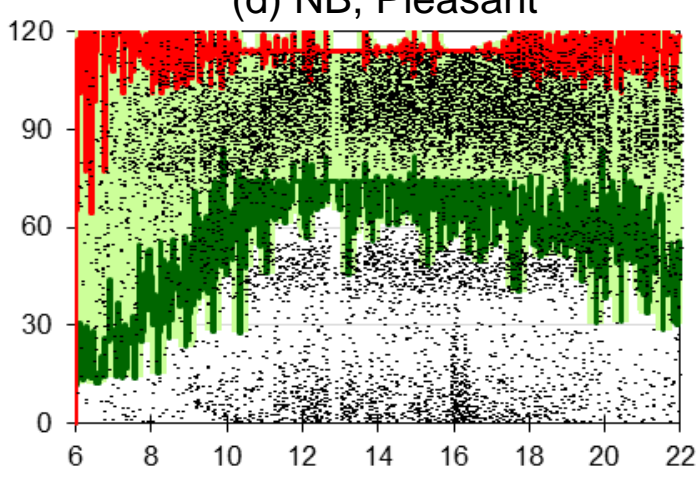

(f) NB, Town \& Country

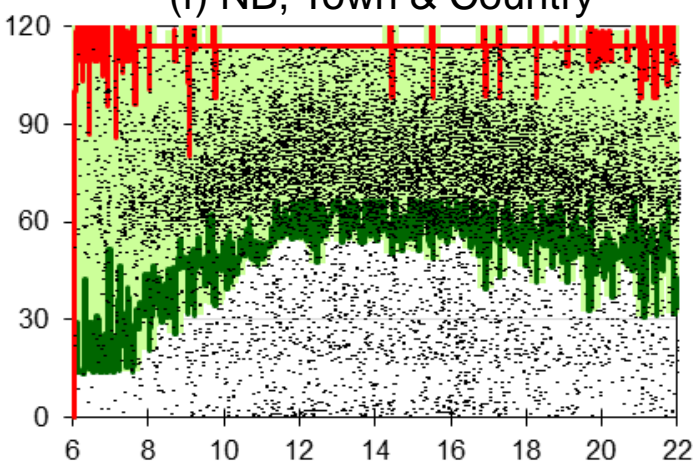

(h) NB, Greenfield

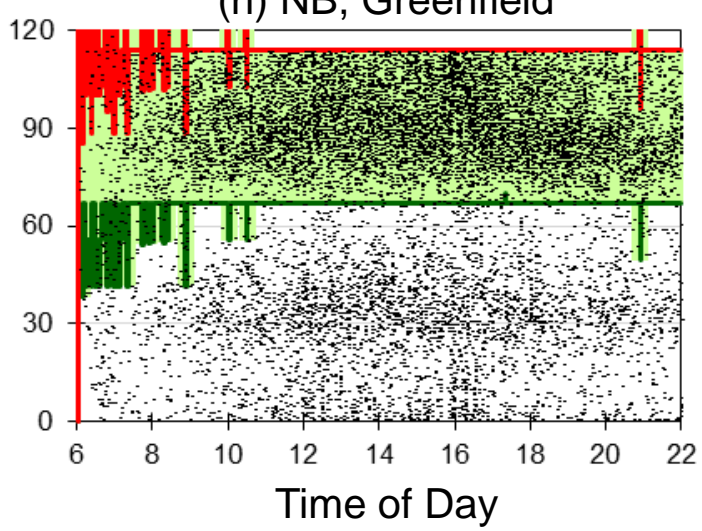

Figure 2.3.4. North End PCDs after 2009 retiming. 


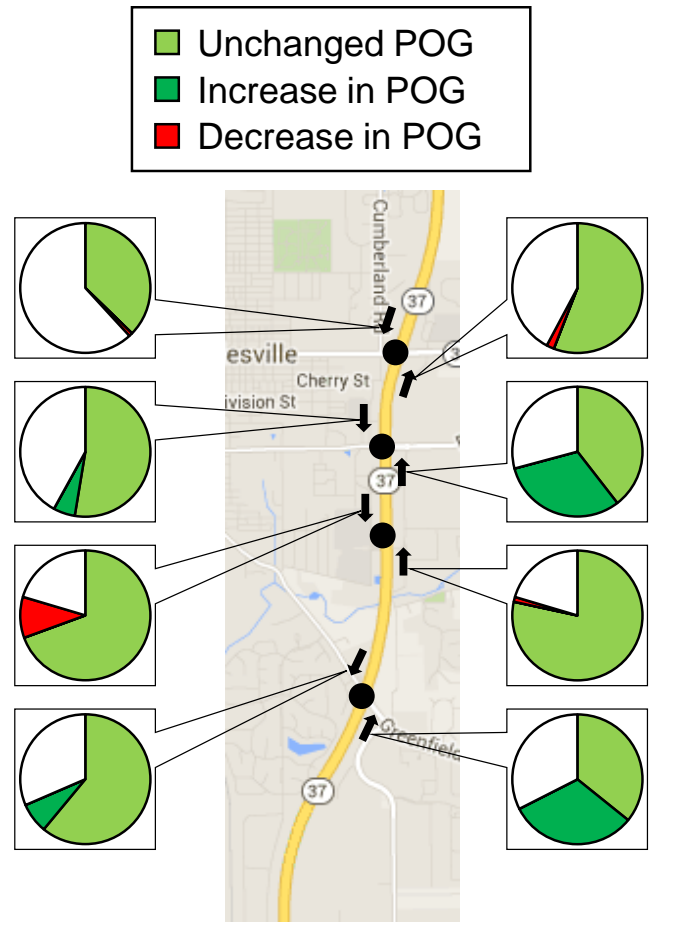

Figure 2.3.5. Summary of changes to POG in 2009.

One year after the initial offset optimization study, in summer of 2010, the four intersections on the south end of SR 37 were equipped with high resolution data logging, and it was decided to repeat the offset optimization process to see whether there would be further improvements on the corridor as a whole. This time, let's consider what happened by first thinking of the changes in POG. Figure 2.3.6 shows a summary view, this time for eight intersections along the corridor. This time, we see small changes in the north end (which had more recently been retimed) while there are drastic changes for some approaches in the south end. Overall, the amount of dark green is far greater than red. There is one approach in particular where the POG seems to increase from $25 \%$ to $75 \%$, a dramatic change in the progression performance. This summary view tells the success story succinctly, but the detailed data that can explain why the numbers changed are revealed in the PCDs. 


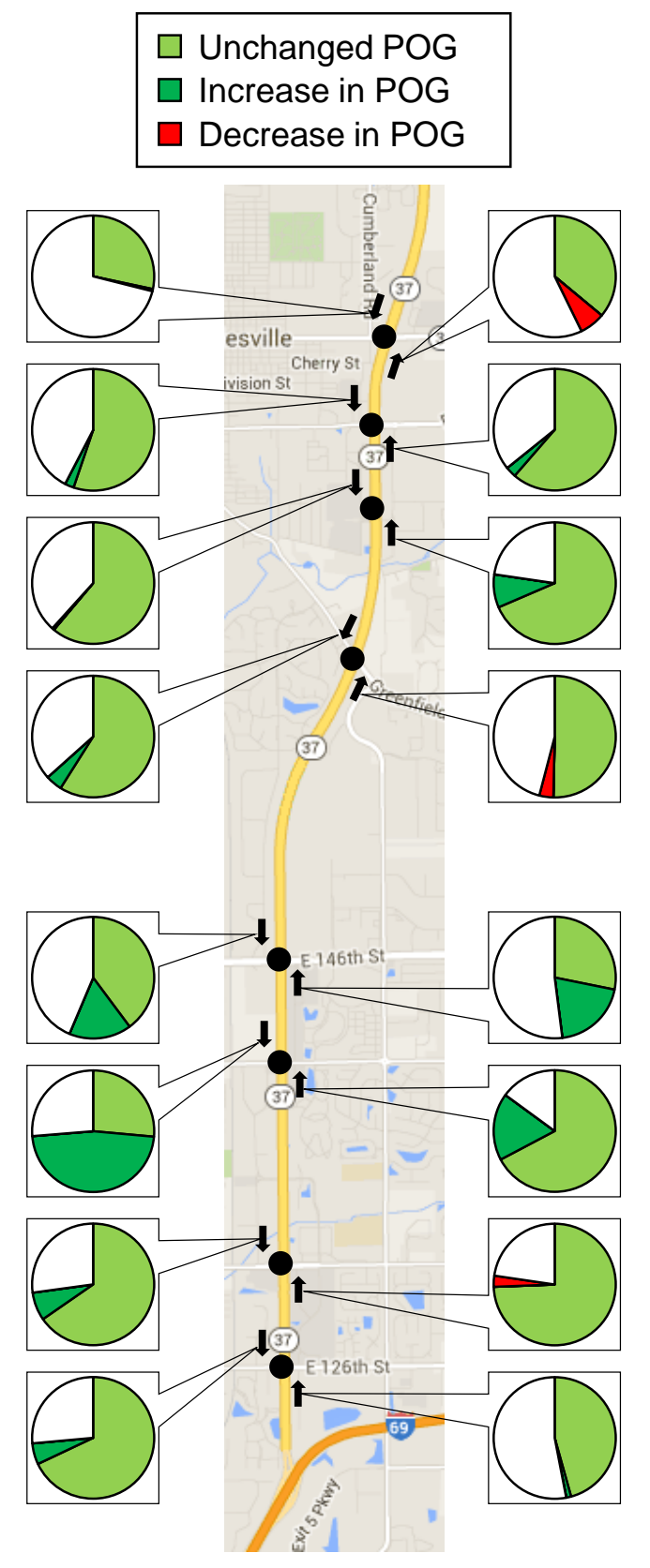

Figure 2.3.6. Summary of changes to POG. 
First, consider the north end of the system. Figure 2.3.7. shows the PCDs for those four intersections prior to optimizing the system in 2010. Visually, these can be compared to Figure 2.3.4, and the arrival characteristics are extremely similar for all eight approaches. That is, within a year's time, there was no substantial change in the arrival characteristics. Figure 2.3.8 shows the PCDs for the north end of SR 37 following optimization of offsets in 2010. The approaches where changes occurred correspond to those with increases or decreases in POG in Figure 2.3.6. Most of the changes are extremely minor. The two most substantial differences are Northbound at SR 32 (Figure 2.3.8b), which got slightly worse, and northbound at Town and Country (Figure 2.3.8f), which a slight improvement.

The south end of the system is where all of the interesting changes took place, and the before/after story here is similar to that of the north end one year previously. Figure 2.3.9 shows the PCDs before optimization of the offsets. In the southbound direction, progression does not appear favorable at $146^{\text {th }}$ St. (Figure 2.3.9a), and is extremely poor at $141^{\text {st }}$ St. (Figure 2.3.9c). However, most of the vehicles do arrive on green at $131^{\text {st }}$ St. (Figure 2.3.9e) and $126^{\text {th }}$ St. (Figure 2.3.9g). In the northbound direction, the first intersection at $126^{\text {th }}$ St. has random arrivals (Figure 2.3.9h), the intersection with $131^{\text {st }}$ St. has arrivals on green (Figure 2.3.9f), the intersection with $141^{\text {st }}$ St. has the end of the platoon cut off during much of the day (Figure 2.3.9d), and the intersection with $146^{\text {th }}$ St. has rather poor progression, with most arrivals occurring during red (Figure 2.3.9b).

Figure 2.3.10 shows the PCDs for a day after the offsets were optimized. Six of the eight approaches show good progression, with most of the arrivals aligned with green, after the offsets were optimized. The other two approaches are the entry into the system, which still has random arrivals as expected (northbound at $126^{\text {th }}$ St., Figure $2.3 .10 \mathrm{~h}$ ), and northbound at $126^{\text {th }}$ St., which has vehicles arriving a little early (Figure 2.3.10b). It would be impossible to adjust offsets to rearrange that arrival pattern without disrupting the opposite direction (southbound at $141^{\text {st }}$ St., Figure 2.3.10c). Therefore, although progression is not completely perfect, it is probably about as close as it can be under the circumstances, when both directions are given consideration. 
(a) SB, SR 32

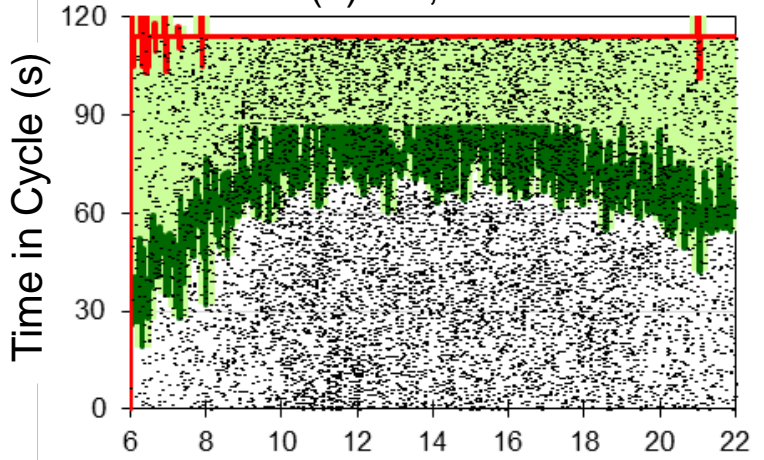

(c) SB, Pleasant

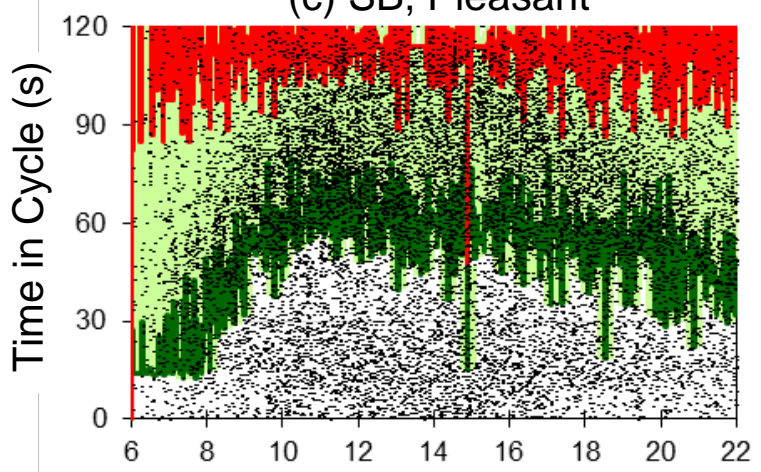

(e) SB, Town \& Country
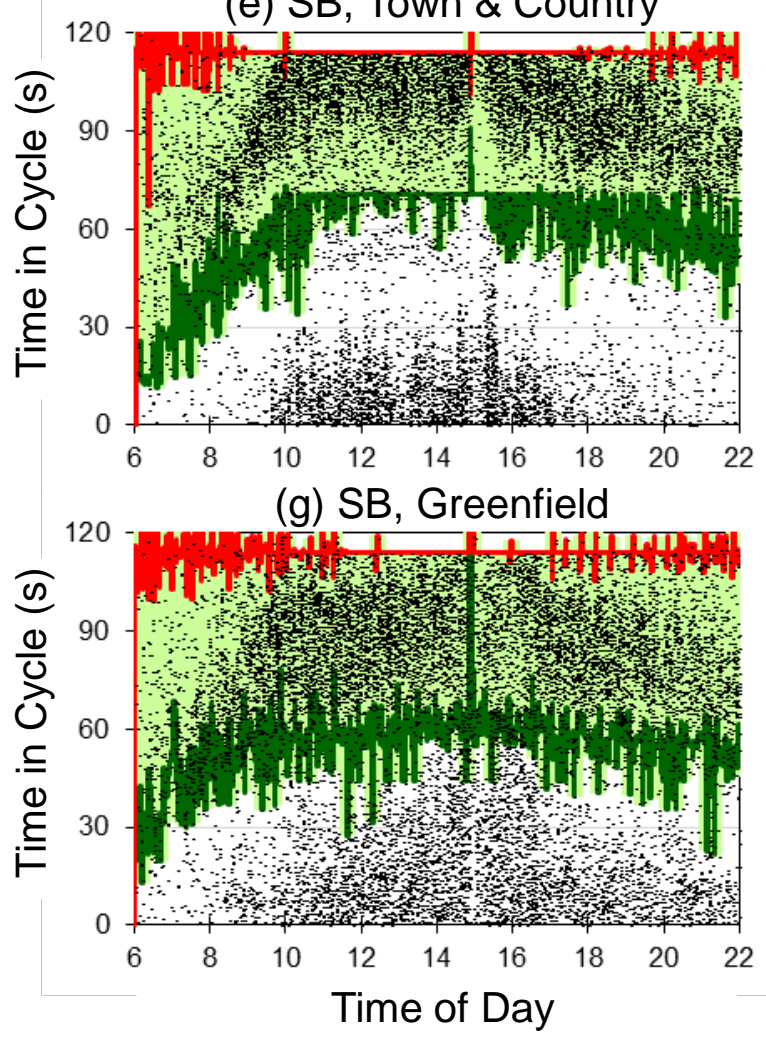

(b) NB, SR 32

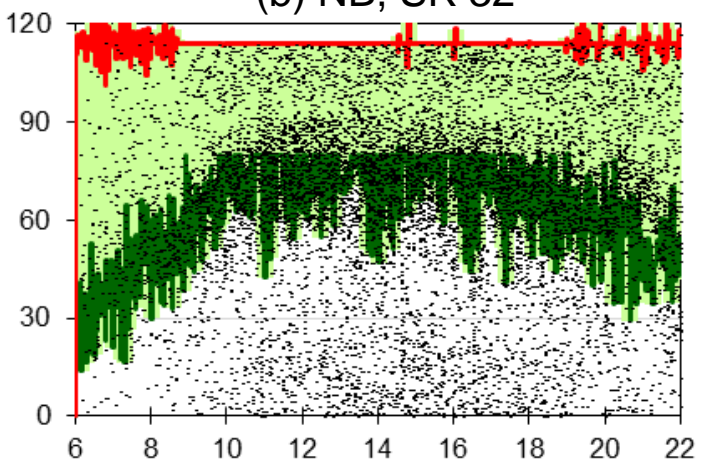

(d) NB, Pleasant

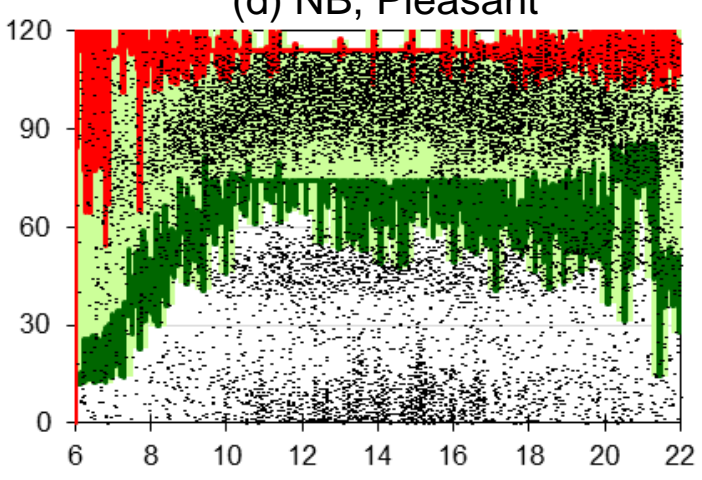

(f) NB, Town \& Country

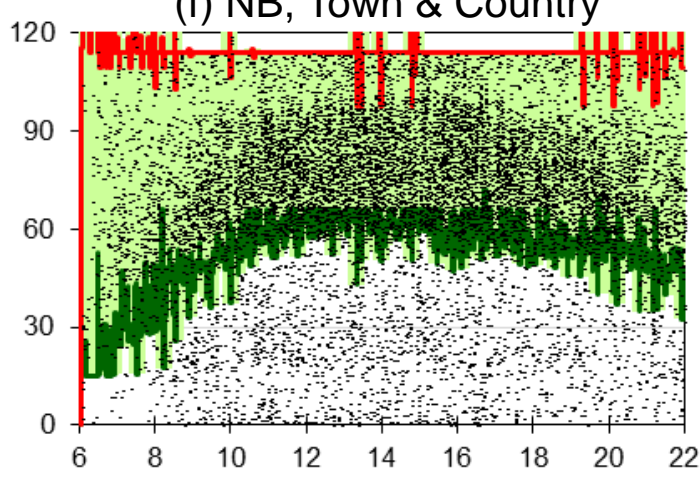

(h) NB, Greenfield

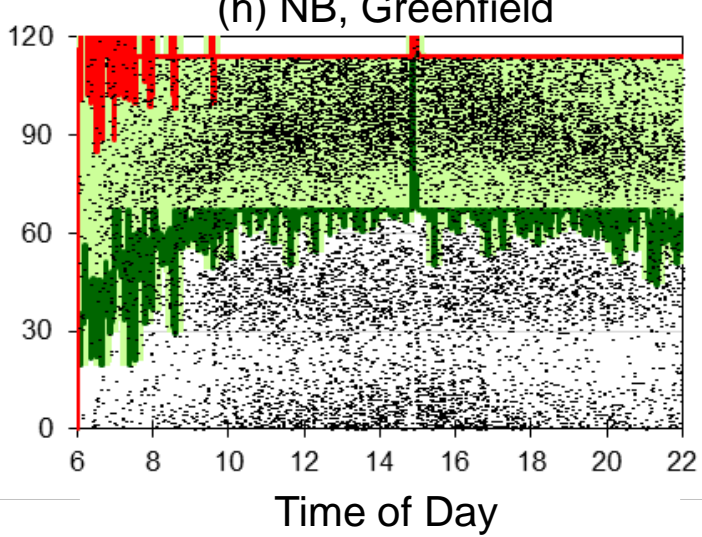

Figure 2.3.7. North End PCDs before 2010 retiming. 
(a) SB, SR 32

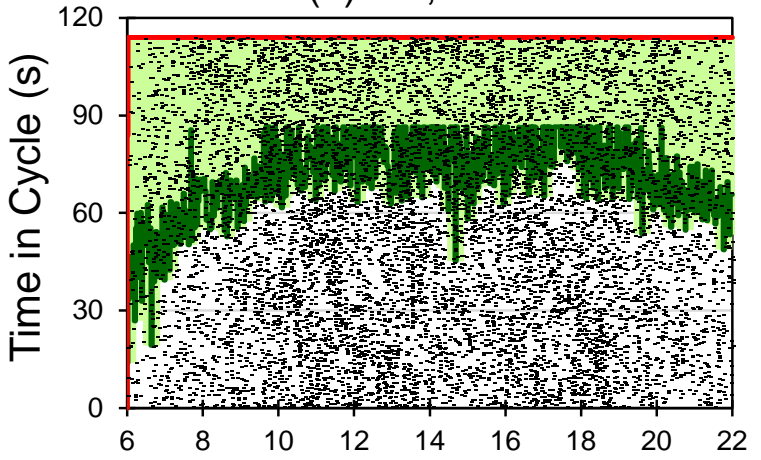

(c) SB, Pleasant

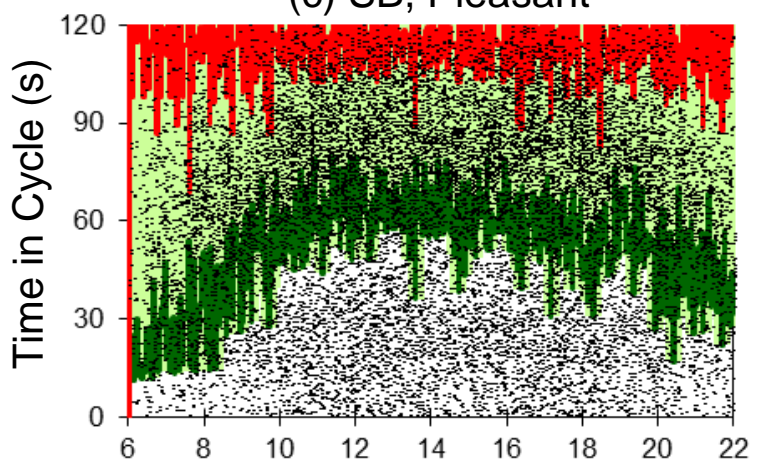

(e) SB, Town \& Country

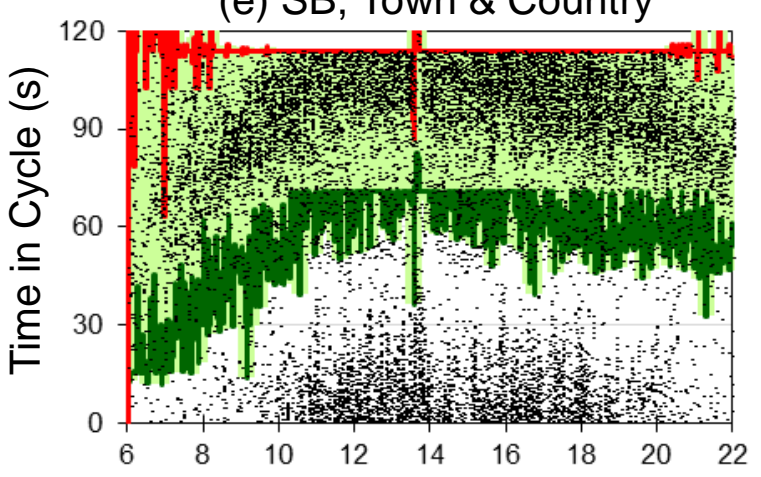

(g) SB, Greenfield

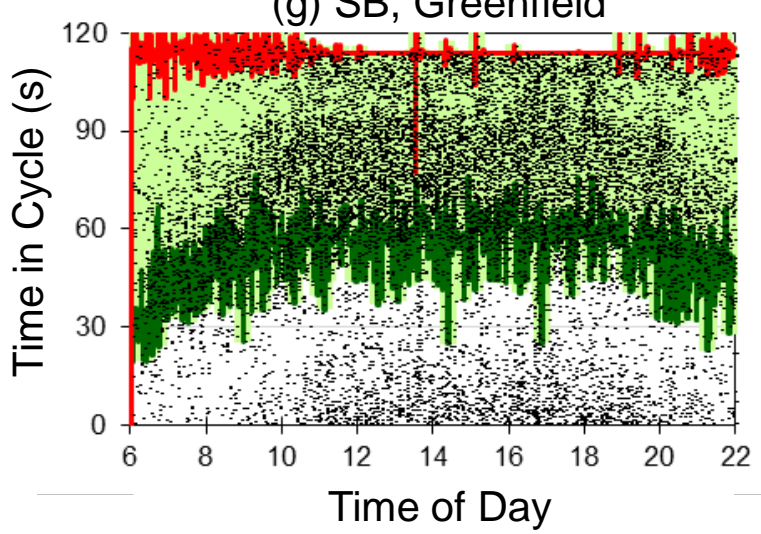

(b) NB, SR 32

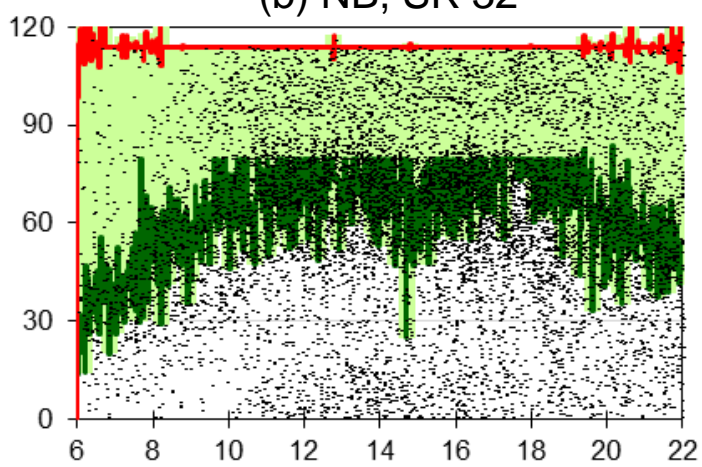

(d) NB, Pleasant

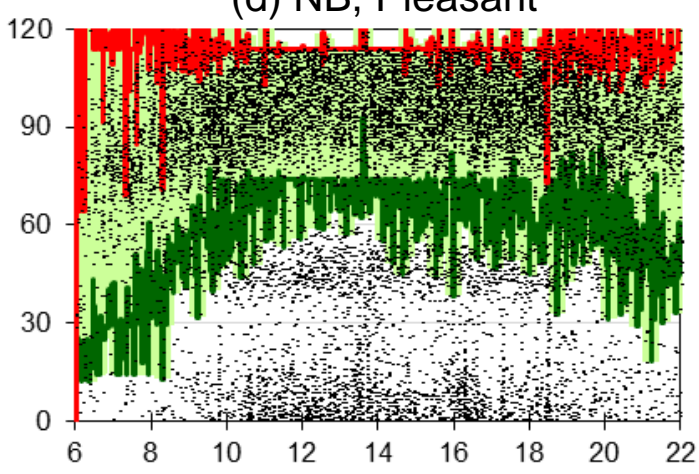

(f) NB, Town \& Country

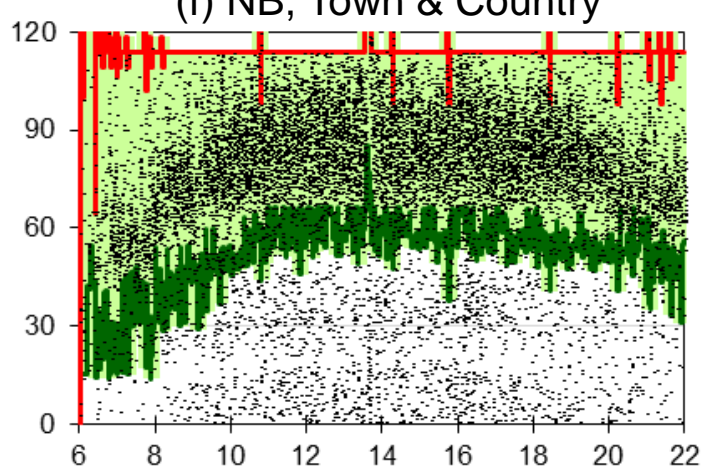

(h) NB, Greenfield

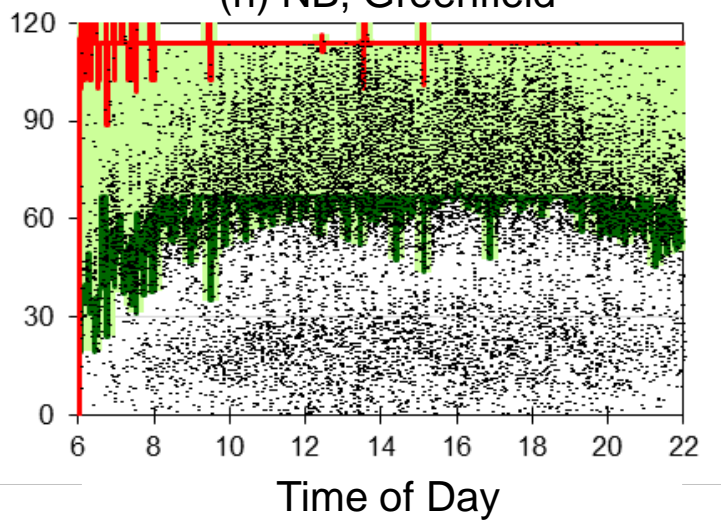

Figure 2.3.8. North End PCDs after 2010 retiming. 
(a) SB, $146^{\text {th }}$ St.

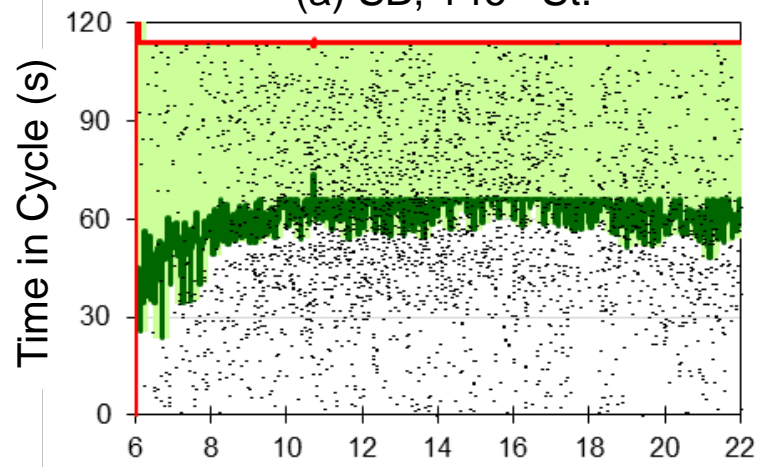

(c) SB, $141^{\text {st }} \mathrm{St}$.

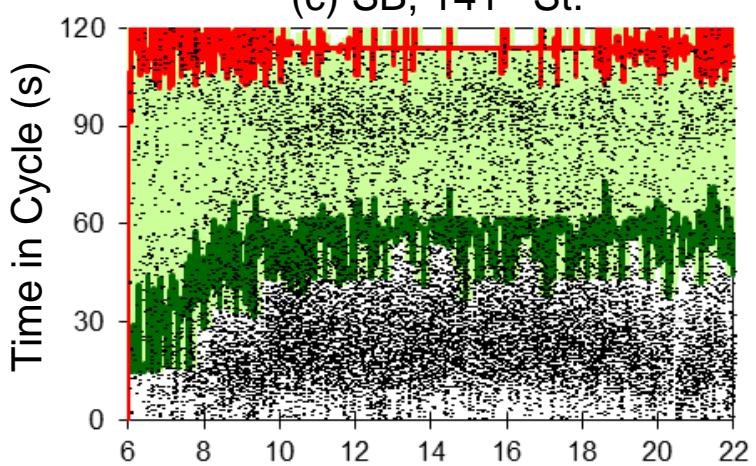

(e) SB, $131^{\text {st }} \mathrm{St}$.

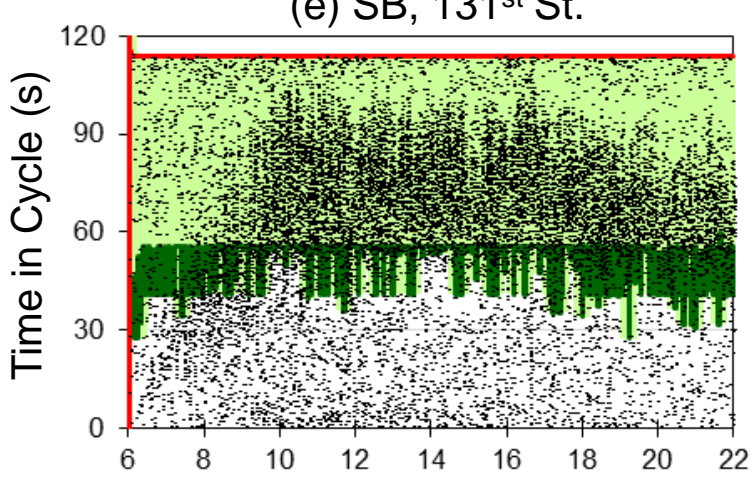

(g) SB, $126^{\text {th }}$ St.

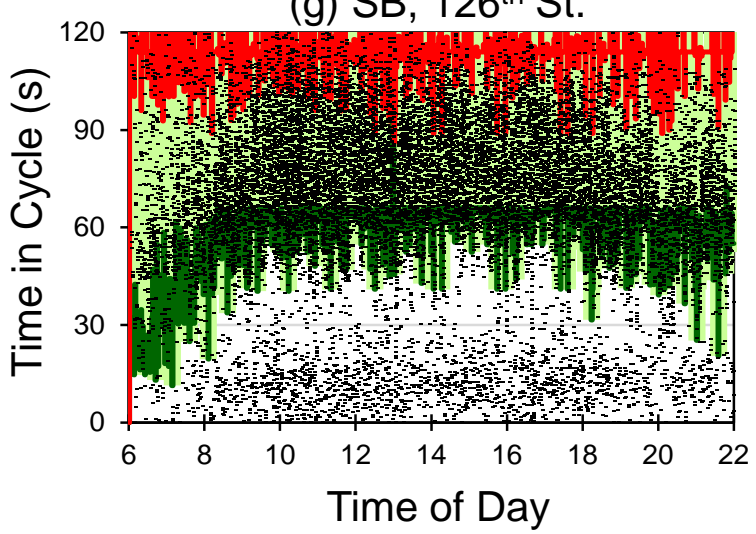

(b) NB, $146^{\text {th }}$ St.

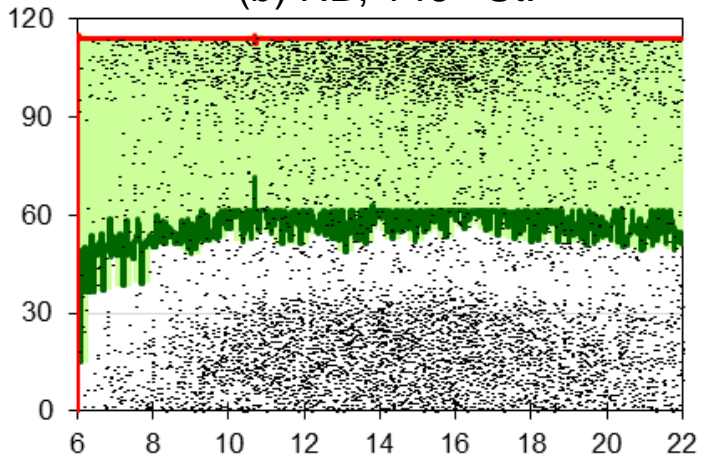

(d) NB, $141^{\text {st }}$ St.

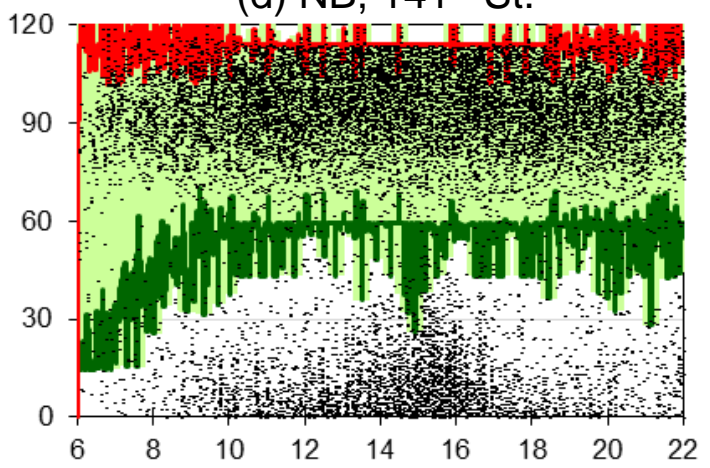

(f) NB, $131^{\text {st }} \mathrm{St}$.

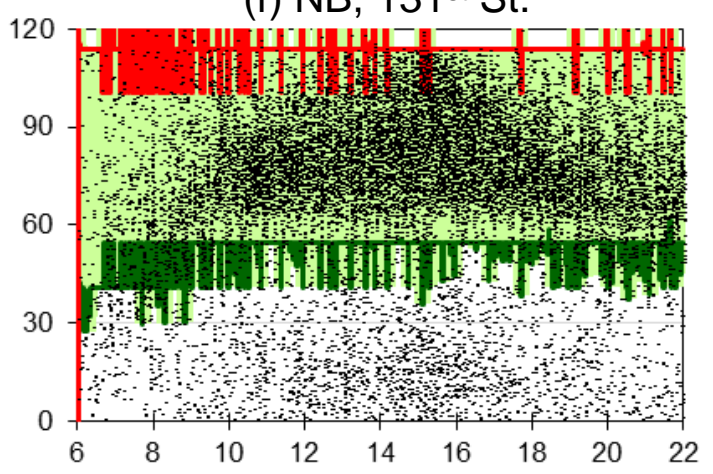

(h) NB, $126^{\text {th }}$ St.

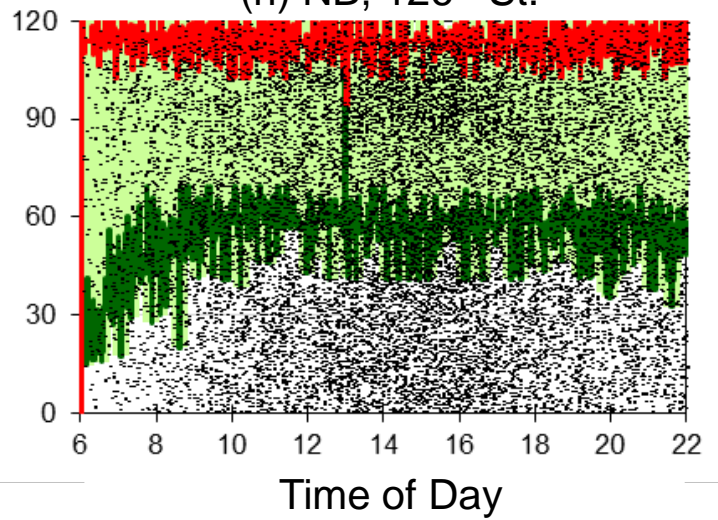

Figure 2.3.9. South End PCDs before 2010 retiming. 
(a) SB, $146^{\text {th }}$ St.

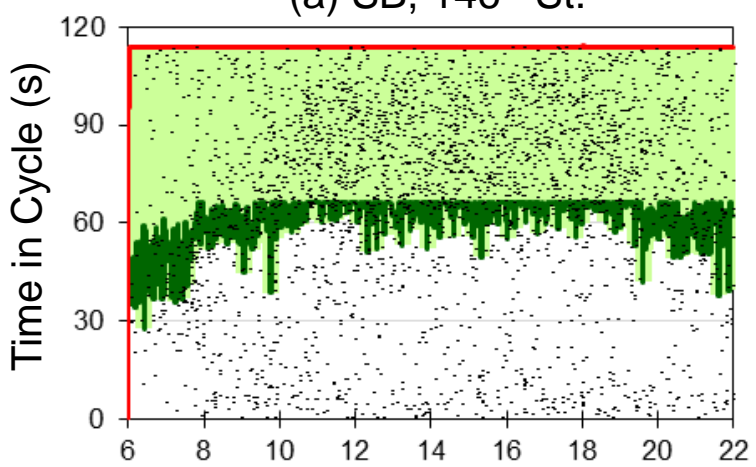

(c) SB, $141^{\text {st }} \mathrm{St}$.

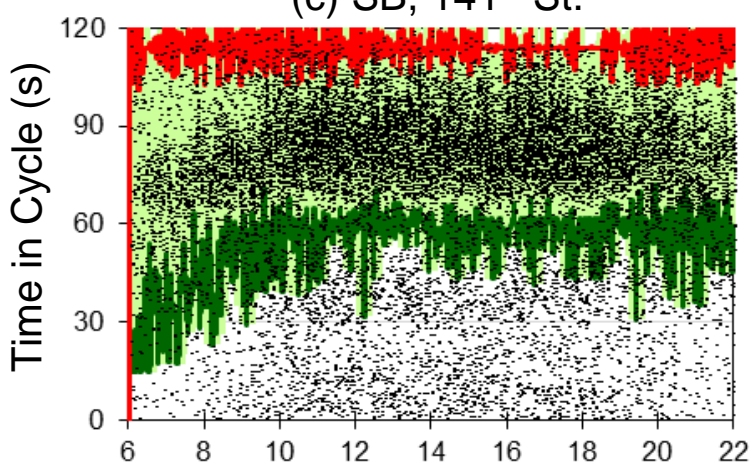

(e) SB, $131^{\text {st }}$ St.

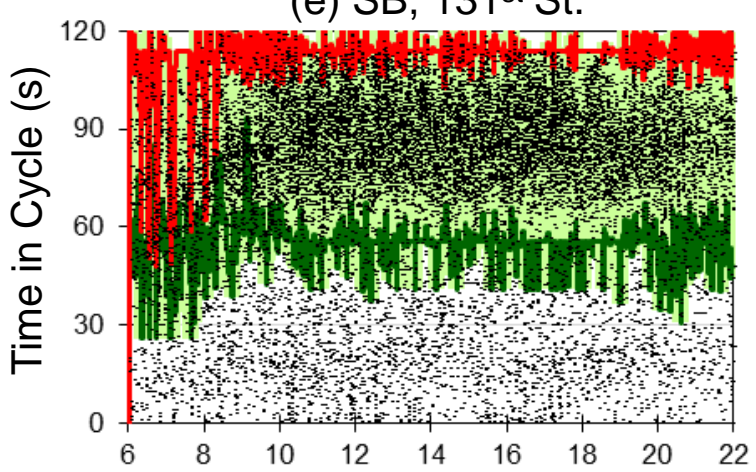

(g) SB, $126^{\text {th }}$ St.

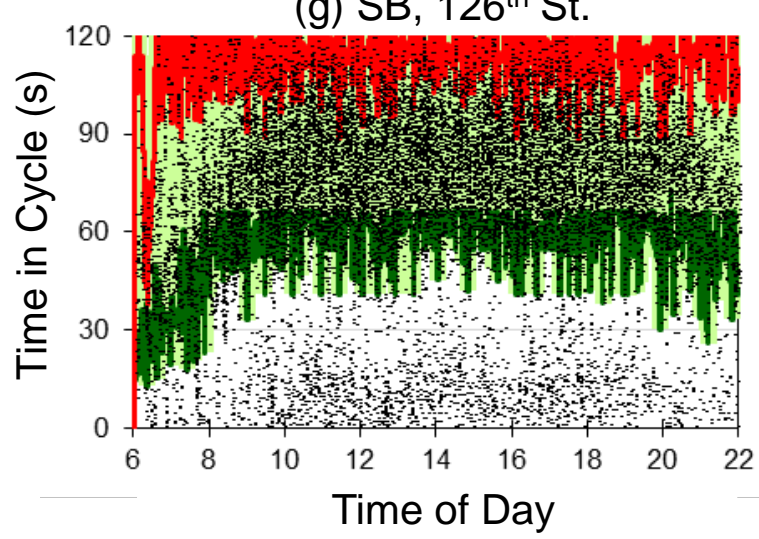

(b) NB, $146^{\text {th }}$ St.

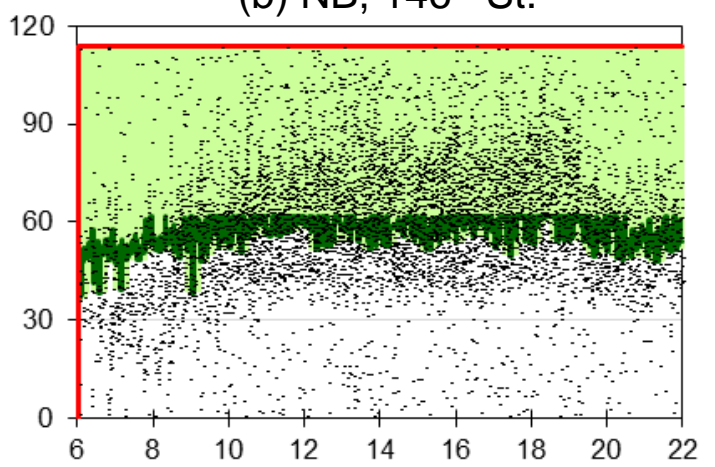

(d) NB, $141^{\text {st }}$ St.

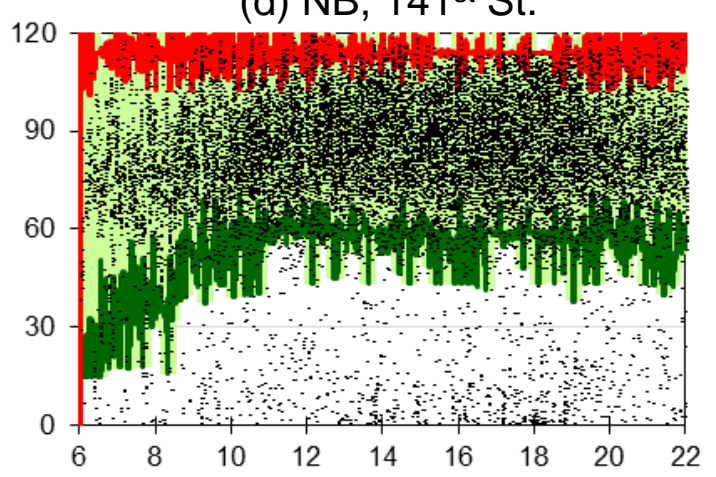

(f) NB, $131^{\text {st }} \mathrm{St}$.

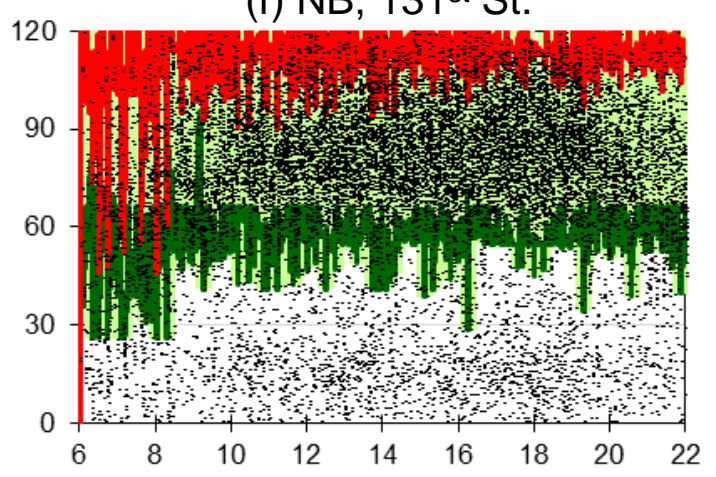

(h) NB, 126 th St.

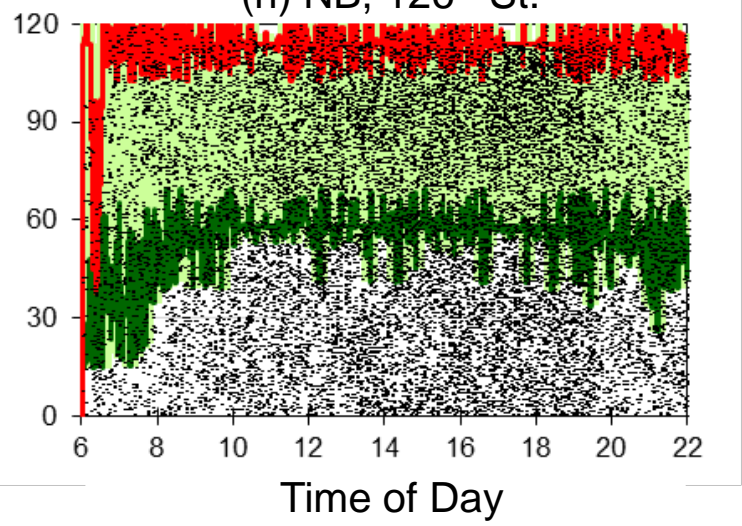

Figure 2.3.10. South End PCDs after 2010 retiming. 


\section{Chapter 3: Capacity Utilization with ROR and GOR Plots}

\subsection{Calculation of ROR and GOR for Determining Split Failure Occurrences}

Capacity allocation, or the distribution of time to competing phases, is one of the primary functions of a traffic signal. The basic idea is to give serve phase in turn, getting to each as quickly as possible, ensure that the phase has enough green time to serve its demand, and then expediently end the green in order to move on to the next phase.

Figure 3.1.1 includes two plots of the flow rate through a signalized approach as the traffic signal cycles through red, green, yellow, and back to red. The bars at the bottom of each plot shows the actual signal displays and the row above it shows the effective red and effective green. As discussed in the previous chapter, the beginning of effective green comes slightly after the actual start of green because of start-up lost time, and the end of effective green comes slightly after the actual end of green, because drivers use a certain potion of the clearance time to continue moving.

Figure 3.1.1a shows the flow rate for an undersaturated phase. After the start of effective green, traffic begins flowing past the intersection at the saturation flow rate. This occurs while any initial queue that accumulated during red is discharged. When the phase is undersaturated, the initial queue will eventually dissipate, and the flow rate will fall under the saturation flow rate to a lower value. This represents randomly-arriving traffic during the later portion of green. By contrast, Figure 3.1.1b shows what occurs when traffic is saturated. Here, rather than diminishing after the initial queue dissipates, the saturation flow rate is sustained through the entire effective green interval. It is very likely that there were some vehicles left behind by the end of green. This situation is called a "split failure," in that the split was not sufficient to serve the traffic.

When assessing the utilization of capacity, it is desirable to know how often the second condition occurs. It is to be expected that during the busiest times of the day, many movements will be inundated with traffic, and it is useful to know the extents of this. It is often difficult to deal with oversaturation during the peak hours. Therefore it is perhaps more useful to know if split failures occur when they are not expected-such as an off-peak timing plan-and if they occur with some opportunity to make an adjustment that can remedy the situation. That is, are there other movements in the same time period that are not having such a severe problem? If so, then rebalancing of the splits may be a potential solution.

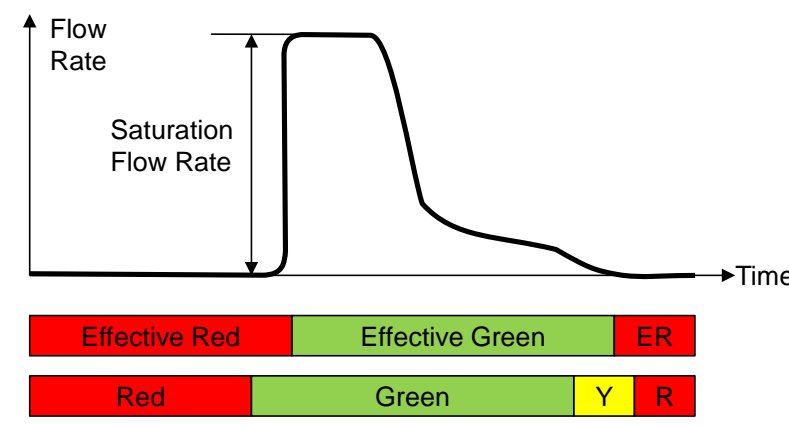

(a) Undersaturated phase.

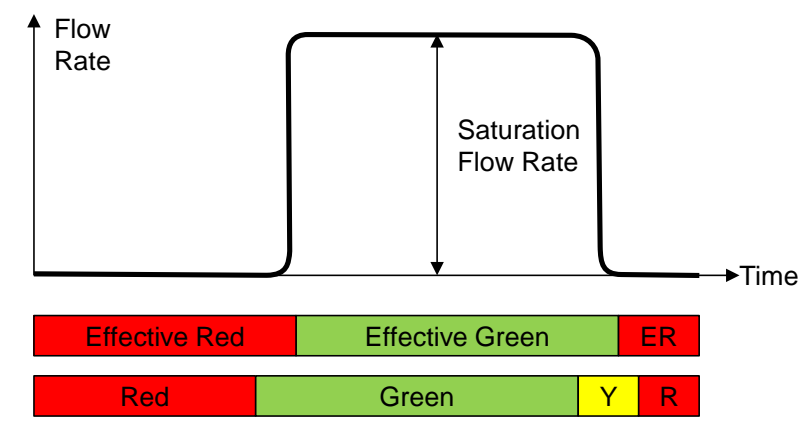

(b) Saturated phase.

Figure 3.1.1. Phase utilization concepts. 
There are several different ways of measuring whether a phase is saturated or not. One way would be to try to measure the demand directly and compare it with the capacity, and compute a volume-to-capacity ratio. One issue with this approach is that it requires a number of assumptions in order to estimate the capacity, and there are many locations where such counts are not easily obtained. However, most actuated signals do feature detectors at the stop bar that are used to call and extend phases. The occupancy of these detectors (whether a vehicle is present or not) can be tracked over time and this can enable performance measures to measure the saturation of the phase.

Figure 3.1.2 shows a plan view of an intersection, US 31 and $126^{\text {th }}$ St. in Carmel, Indiana, which will serve as a test site for developing a metric based on detector occupancy. The examples will focus on the eastbound approach. There is a left turn phase with one lane, and a through-right turn phase with two lanes.

First consider the eastbound left turn, which is undersaturated. Figure 3.1.3 shows an example cycle for this phase, showing the detector occupancy over time, and the status of the phase over time. Two camera views are included for this cycle showing the conditions in the field just after the start of green and 5 seconds after the start of red. At the start of green, there are two vehicles in the left-turn lane; these depart early into the green and the occupancy drops off; the phase then terminates, going through yellow and to red, and there is no further occupancy until the next vehicle arrives well into the next cycle. Note the row labeled "occupancy ratios" in Figure 3.1.3; these blocks shows the proportion of certain intervals during which the detector was occupied. The green occupancy ratio (GOR), corresponding to the entire green interval, is $67 \%$, or in other words, the detector was occupied during $67 \%$ of the green. $\mathrm{ROR}_{5}$ represents the red occupancy ratio of the first five seconds (which we will simply call "ROR" for short), which was $0 \%$. The detector was unoccupied for the first five seconds of red.

If we take these two pieces of information and develop a plot of ROR versus GOR for all of the cycles during the same time of day, it becomes possible to see the range of performance. Figure 3.1.4 shows such a plot for this undersaturated left turn phase. Each icon represents an individual cycle; note that virtually all of the cycles fall on the ROR $=0 \%$ line, meaning that there were no vehicles present after the end of green. Some cycles do have nonzero values, but there are likely due to vehicles arriving late in the cycle rather than leftover queued demand. This assertion can be made because the corresponding GOR values within the same cycle are also low, meaning that there had to have been significant gaps in the flow at times during green. 


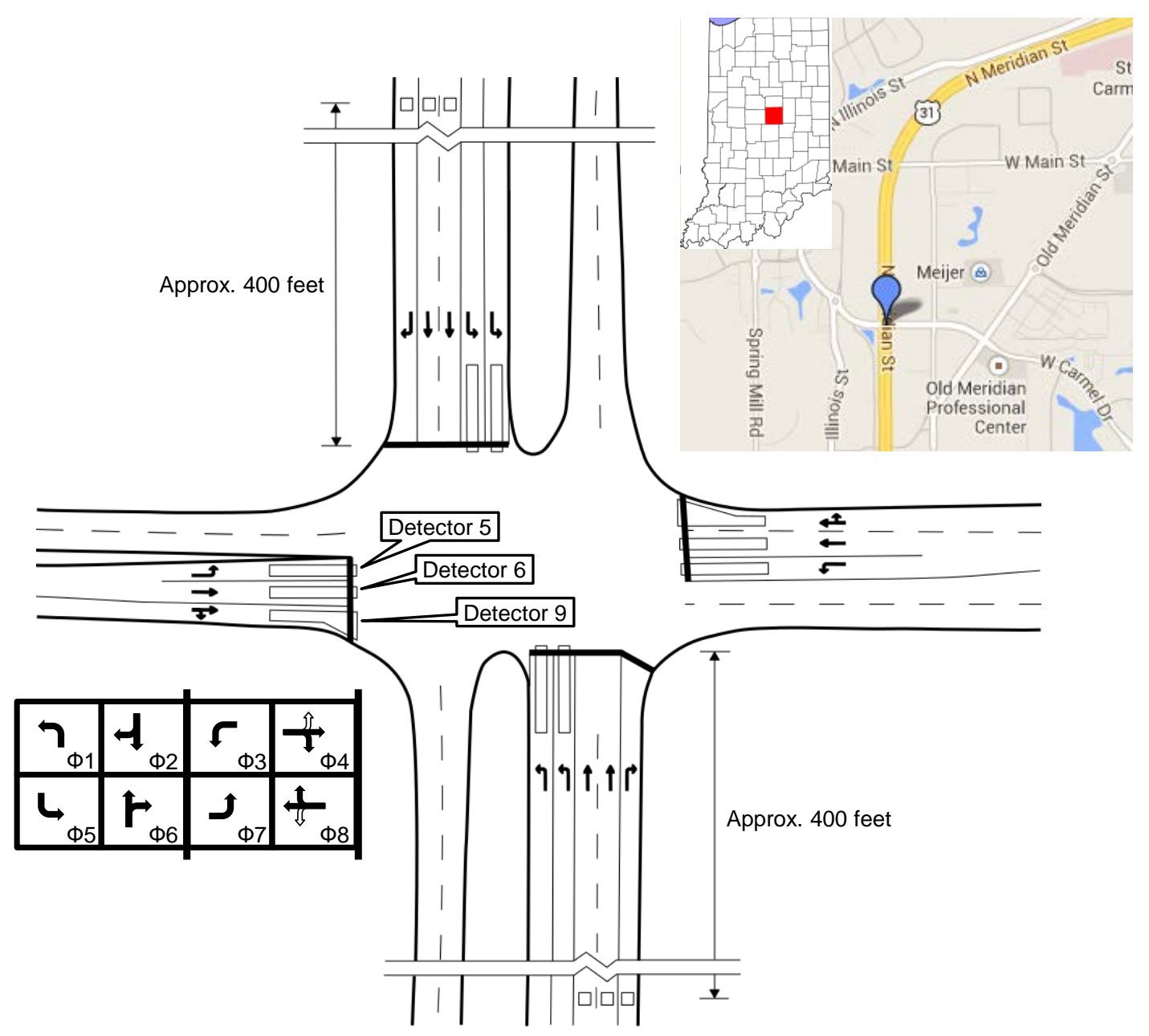

Figure 3.1.2. Phase and detector layout, US 31 and $126^{\text {th }}$ St, Carmel, IN. 


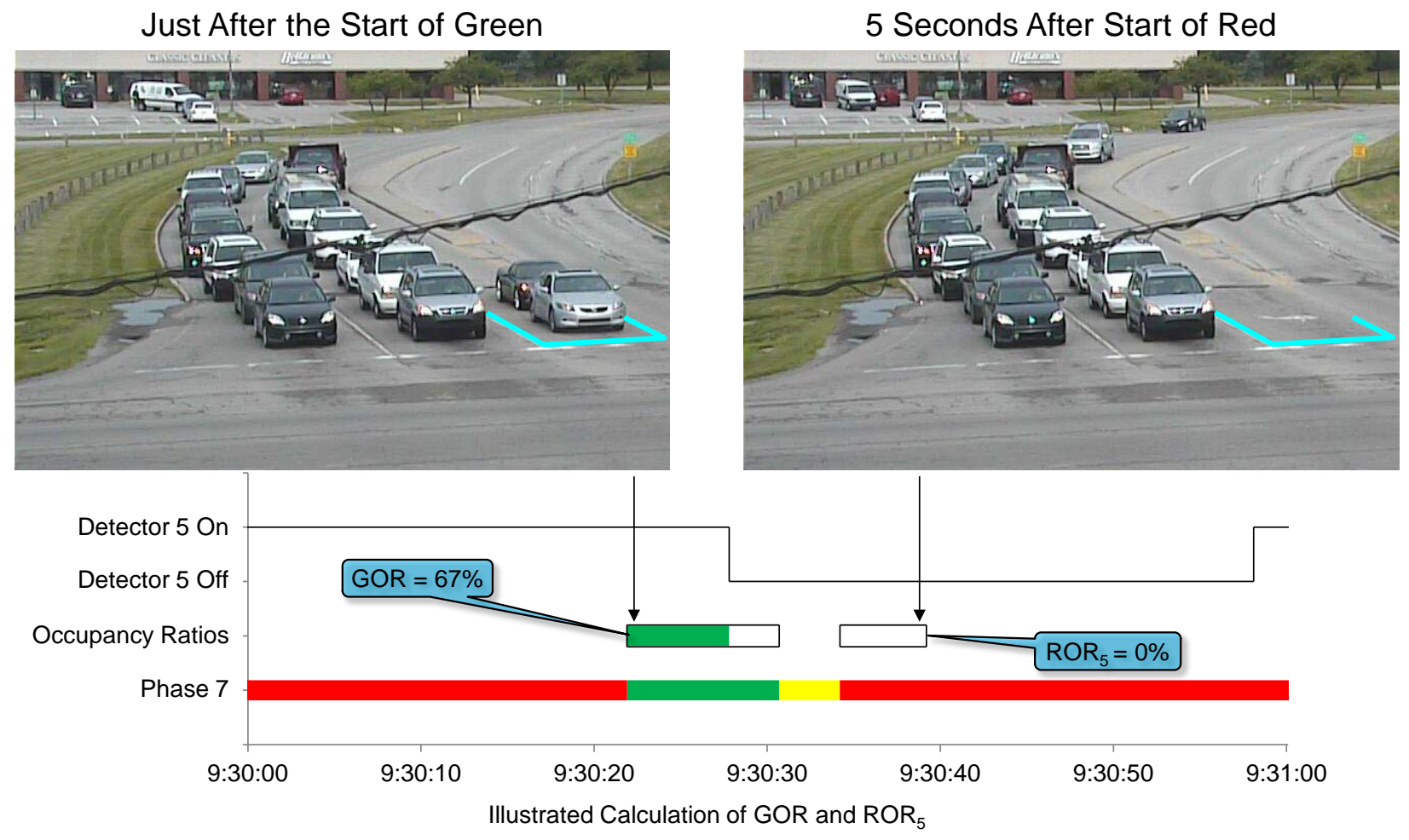

Figure 3.1.3. Example computation of ROR and GOR: undersaturated phase.

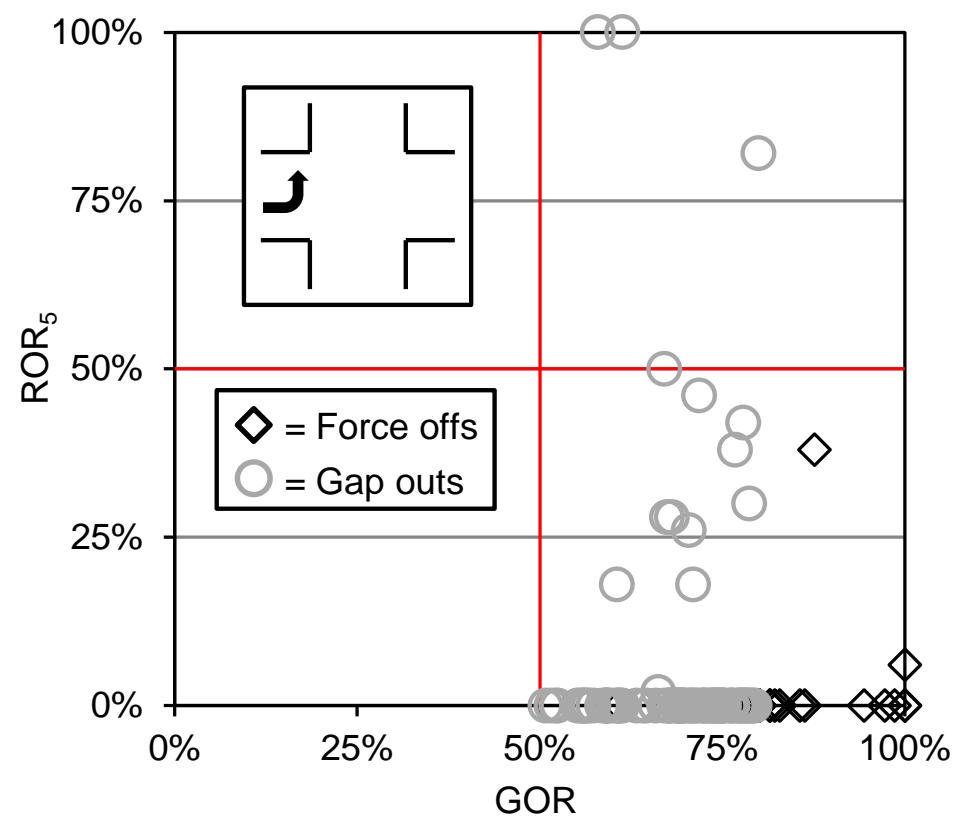

Figure 3.1.4. Plot of ROR/GOR for an undersaturated phase (0900-1500). 
Now let us consider the eastbound through movement, which is saturated. From the video images in the previous example, the extent of the queue seemed to be proof enough. How does the data appear for a phase with this condition? Figure 3.1.5 shows a similar diagram for the two through lanes and how their GOR and ROR appear during an example cycle. The video image just after the start of green shows a very long queue; note the red highlighted vehicle in the queue. The video image from 5 seconds after the start of red shows that this particular vehicle did not clear the intersection, and there are others behind it.

The two detectors in the two lanes chatter on and off as traffic flows during green, but their collective occupancy is completely solid. The detectors are completely occupied during green, so GOR $=100 \%$. During the first five seconds of red, there is a little chatter as the traffic begins settling into position, but the ROR is still high, at $90 \%$. Together, these numbers indicate that the phase was fully utilized during the entire green, and there was some leftover demand following the start of red. This indicates the occurrence of a split failure.

Figure 3.1.6 shows how the ROR and GOR values appear in a plot of all the individual cycles within the same time of day plan. Note now that there are many of the values clustered in the upper right hand corner, where GOR and ROR are both high. There is considerable variation throughout the time period, but it is evident that this phase has a capacity problem that certainly seems to affect a large number of cycles. Compare this with Figure 3.1.4, which exhibits hardly any cycles at all inside of that upper right hand corner.

This graphical tool, based on quantitative analysis of the event data, provides a means of evaluating the quality of capacity allocation on a given phase. To summarize the performance in a single number, one option would be to count the number of cycles in the upper right-hand corner - the number of likely split failures. To do so, we can select some threshold values above which to regard a cycle as being in that range of values. In this study, we used values of ROR $=80 \%$ and GOR $=80 \%$ as a starting point. Using these thresholds, we find that the left turn movement (Figure 3.1.4) experienced 1 split failure, while the through movement (Figure 3.1.6) experienced 80 split failures.

The next step is to begin rolling up this analysis to the intersection level and beyond, to begin identifying where capacity deficiencies exist within the system. 
Just After the Start of Green

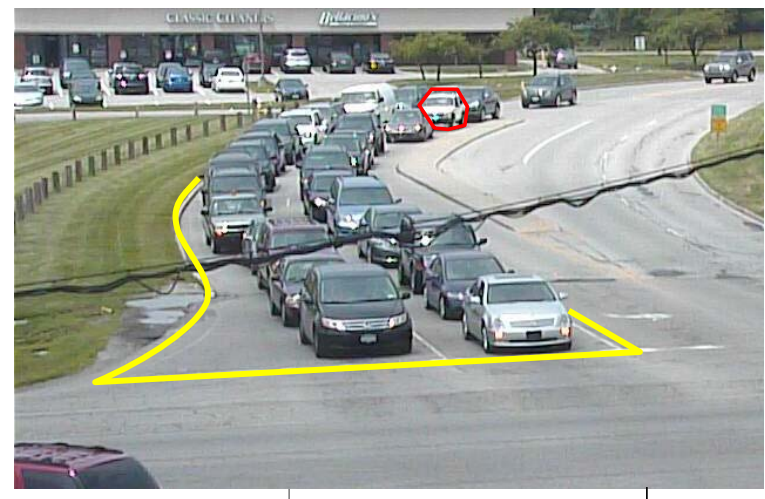

5 Seconds After Start of Red

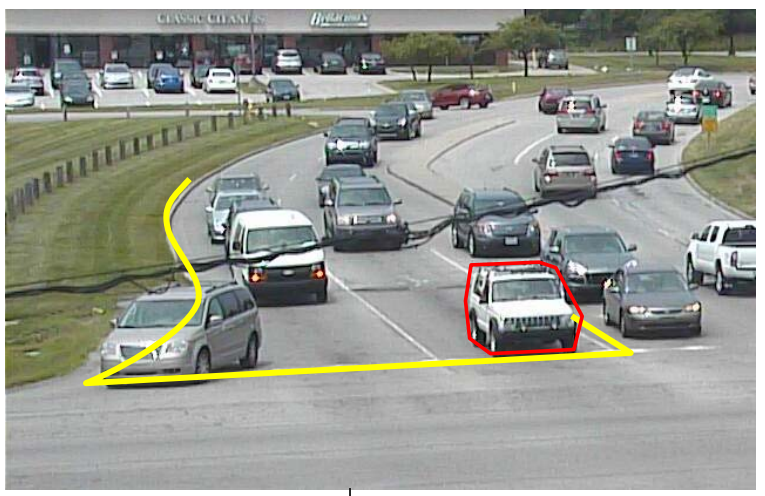

Detector 6 On

Detector 6 Off

Detector 9 On

Detector 9 Off

Detector (6 or 9) On

Detector (6 and 9) Off

Occupancy Ratios

Phase 4

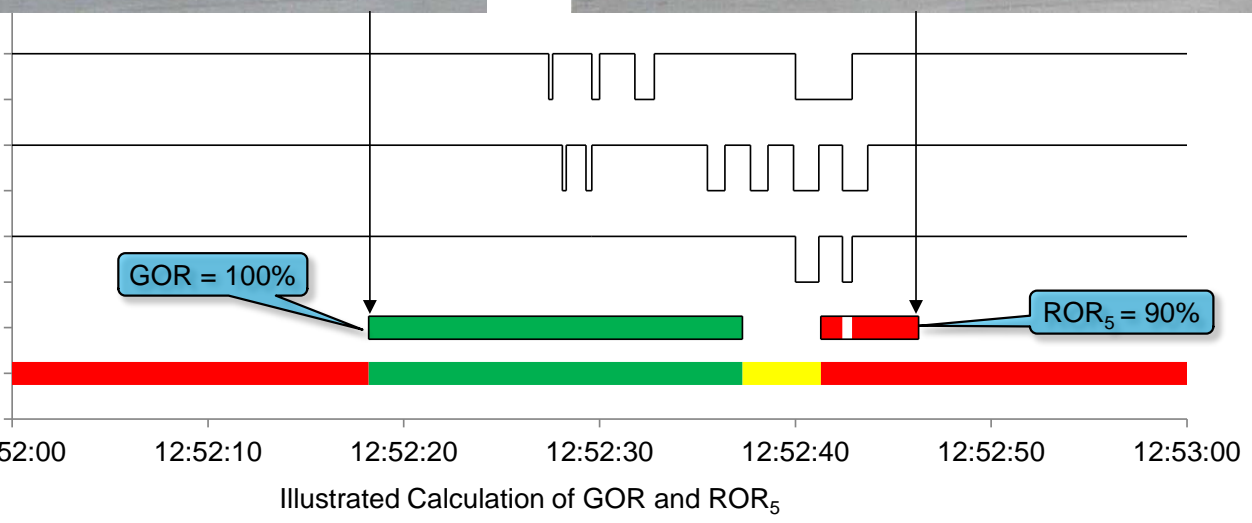

Figure 3.1.5. Example computation of ROR and GOR: oversaturated phase.

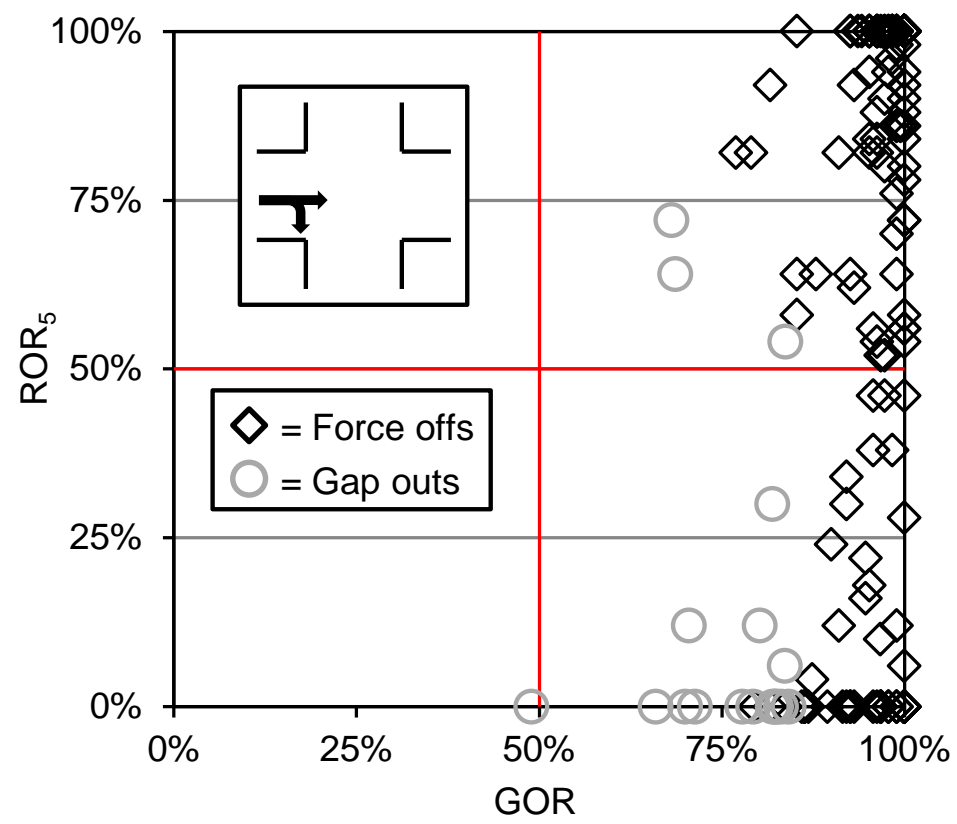

Figure 3.1.6. Plot of ROR/GOR for an oversaturated phase (0900-1500). 


\subsection{Intersection and Corridor Analysis of Split Failures}

The previous section introduced the concepts of ROR and GOR and how together they can be used to estimate the occurrence of split failures. It is now worth considering the performance of the entire intersection - all of its eight phases - during the same time period to determine which phases are having problems and how these might be corrected.

Figure 3.2.1 contains a plot of ROR versus GOR for all eight phases at the intersection, including the count of the number of cycles thought to be split failures (that is, whose ROR and GOR values both are in excess of 80\%).

First of all, looking back at Figure 3.1.2, we might remember that phases 2 and 6, the "mainline" through movements, do not have any detectors at the stop bar. The only available detectors for these phase are the advance detectors, which are well upstream. Thus, the ROR and GOR data for these two phases are invalid, as they have nothing to do with the departing traffic. On the other hand, because of the advance detection, it is possible to obtain a count of the arriving traffic and, from this, to calculate the volume-tocapacity (v/c) ratio. Plots of those are shown for phases 2 and 6 in Figure 3.2.1i and j respectively.

As for the other phases, it is clear that there are many which have substantial numbers of split failures during the midday time period. Phases $1,3,4$, and 8 each have many split failures, while phases 5 and 7 have few. In the meanwhile, phases 2 and 6 both have rather low rates of utilization, with v/c ratios hovering around $50 \%$ for most of the timing plan. This means that phases 2, 5, 6, and 7 are potential candidates for possibly giving up green time.

In this case, it was decided to remove an amount of split equal to $4 \%$ of the cycle from phases 2 and 6 , and redistribute it to phases 3 and 8. By doing so, it was hoped to improve operations on those phases directly. It could also be hypothesized that, if there were any cycles where phase 3 terminated early, the extra split might also benefit phase 4 .

The impact on phase 8 are illustrated in Figure 3.2.2 and 3.2.3, which respectively show the ROR/GOR plots before and after the split adjustment. Initially, there are many points in the upper right hand corner of the ROR/GOR plot, as Figure 3.2.2 shows, meaning that there are many split failures. After the split adjustment, the cluster of points has moved away from that region, as shown in Figure 3.2.3. There are still some split failures, but overall there are considerably fewer. 


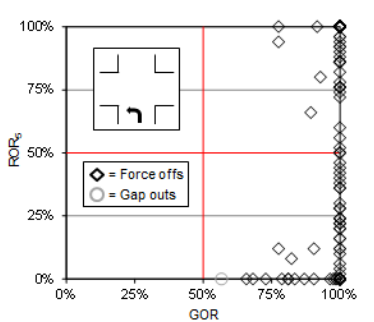

a) $\phi 1 \mathrm{ROR} / \mathrm{GOR}$ (61 split failures)

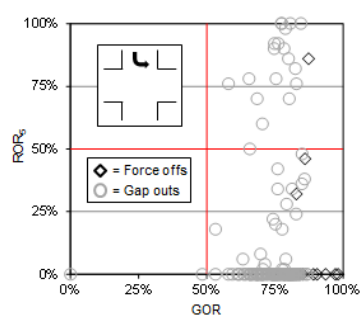

e) $\$ 5 \mathrm{ROR} / \mathrm{GOR}$ (3 split failures)

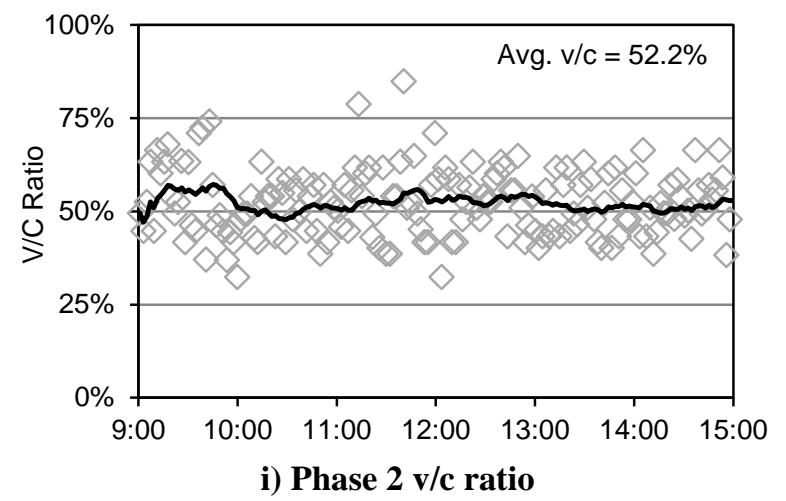

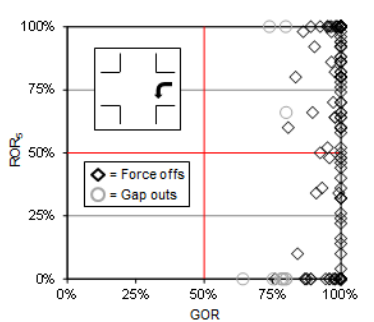

c) $\$ 3 \mathrm{ROR} / \mathrm{GOR}$ (116 split failures)

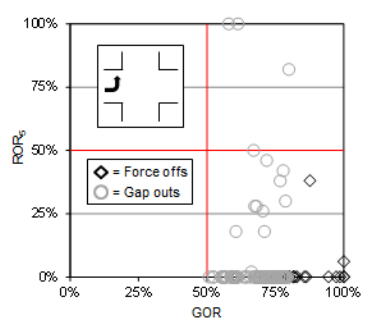

g) $\$ 7 \mathrm{ROR}_{5}$ vs. GOR (1 split failure)

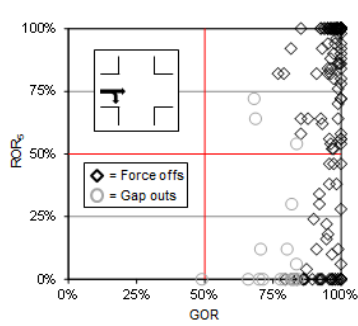

d) $\$ 4 \mathrm{ROR} / \mathrm{GOR}$ (80 split failures)

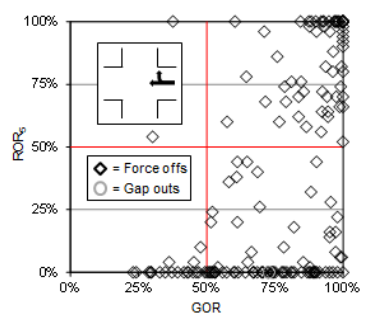

h) $\$ 8 \mathrm{ROR} / \mathrm{GOR}$ (43 split failures)

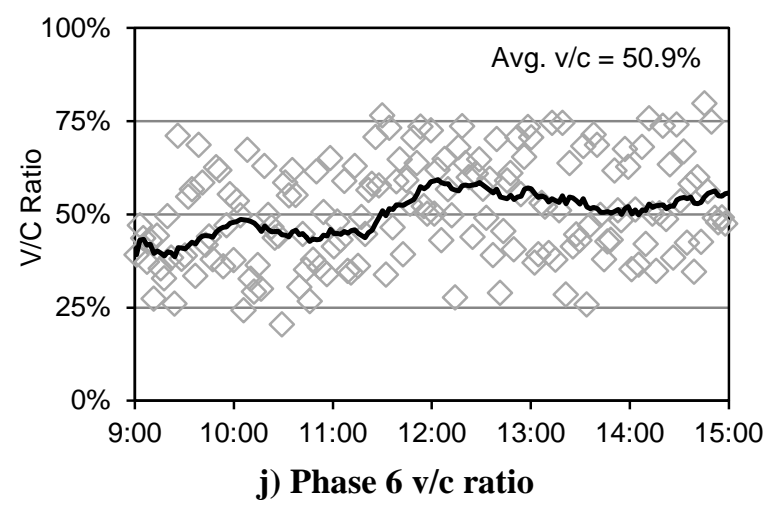

* Phase 2 and Phase 6 ROR $_{5}$ and GOR were calculated based on advanced detectors and therefore not a valid measurement of capacity utilization. The resulting data is included for illustrative purposes.

Figure 3.2.1. Split failure analysis: A plot of raw ROR and GOR values for eight phases, and v/c ratio for phases 2 and 6, for the 0900-1500 time period. 
To view the impact on all eight phases, we will examine how the total number of split failures changed, which succinctly quantifies the impact. Figure 3.2.4 shows a series of bar graphs arranged in an eightphase configuration, showing the number of split failures before and after the split adjustment. Phases 2 and 6 have no apparent split failures in either case, as their v/c ratio is below 1 for all cycles before and after the split adjustment. The other phases show split changes as measured using stop bar occupancy.

As expected, phases 3 and 8, which received additional green time, both had reductions in the number of split failures. Phase 3 saw the number reduce from 116 to 52, while phase 8 went from 43 to 15 . Phase 4, in the meanwhile, had about the same number of split failures. Earlier, we had speculated that phase 4 might absorb any unused additional green time given to phase 3, but clearly not enough time carried forward to make a difference on phase 4. Most likely, the split of phase 4 would need to be increased directly to have an impact. Phase 1, in the meanwhile, saw a slight increase in the number of split failures, which may be due to an increase in demand since its split was unchanged, and phases 5 and 7 had no differences.

Another way to look at the split failure data is to consider not only the frequency of split failures, but also their severity. It might be argued, for example, that the occasional split failure is tolerable as long as the traffic can be cleared in the following cycle. A more serious problem arises when split failures repeat for many cycles in a row, so that there is unmet demand for a longer time period, and queues are likely to be continuously growing. One way to examine this is to consider all of the cycles in their time order and look at when there are 3 or more cycles in succession which all have split failures.

Figure 3.2.5 shows the count of cycles where the number of consecutive split failures was 3 or higher, for all eight phases. As before, there were no split failures on phases 2 and 6 . The other phases follow a similar trend as before, except that we now see that phase 8 went from having 6 occurrences of three or more failures in a row, to zero occurrences; and phase 3 went from 27 to 4.

These two visualizations would be helpful to demonstrate the impact of making a specific intervention such as a split adjustment, as well as to verify that the change produced the desired effect. In this case, the phases receiving green time saw improvements, as expected, while the phases that gave up the green time did not see worsened performance. 


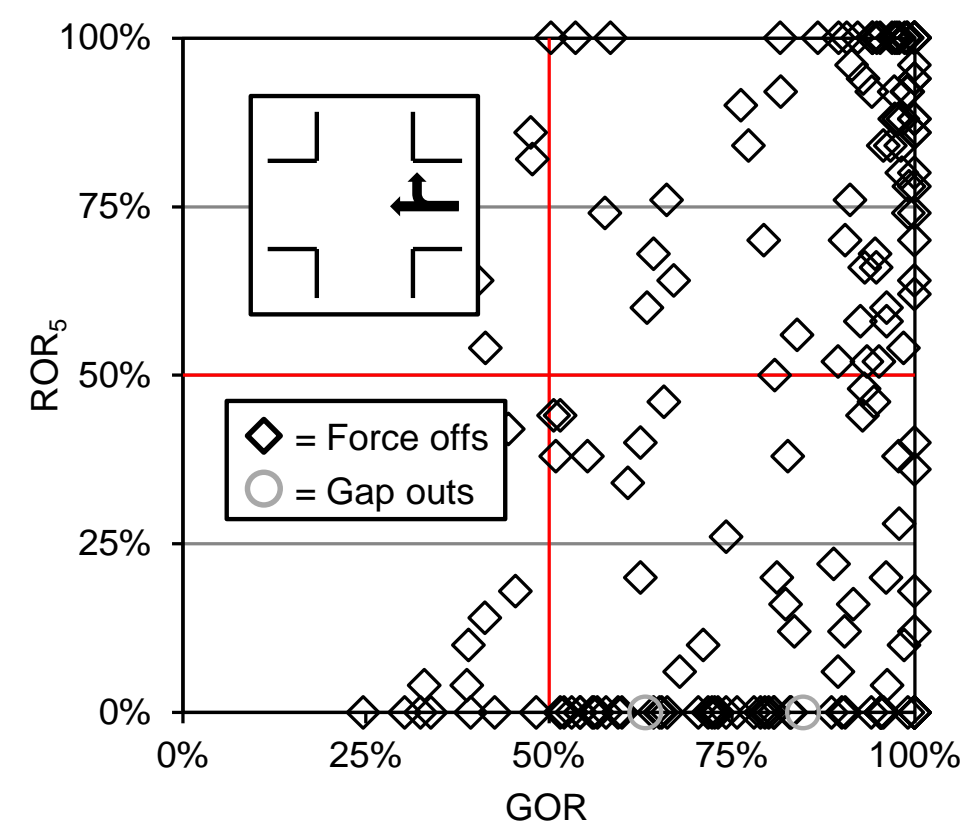

Figure 3.2.2. Phase 8 ROR/GOR plot before split adjustment.

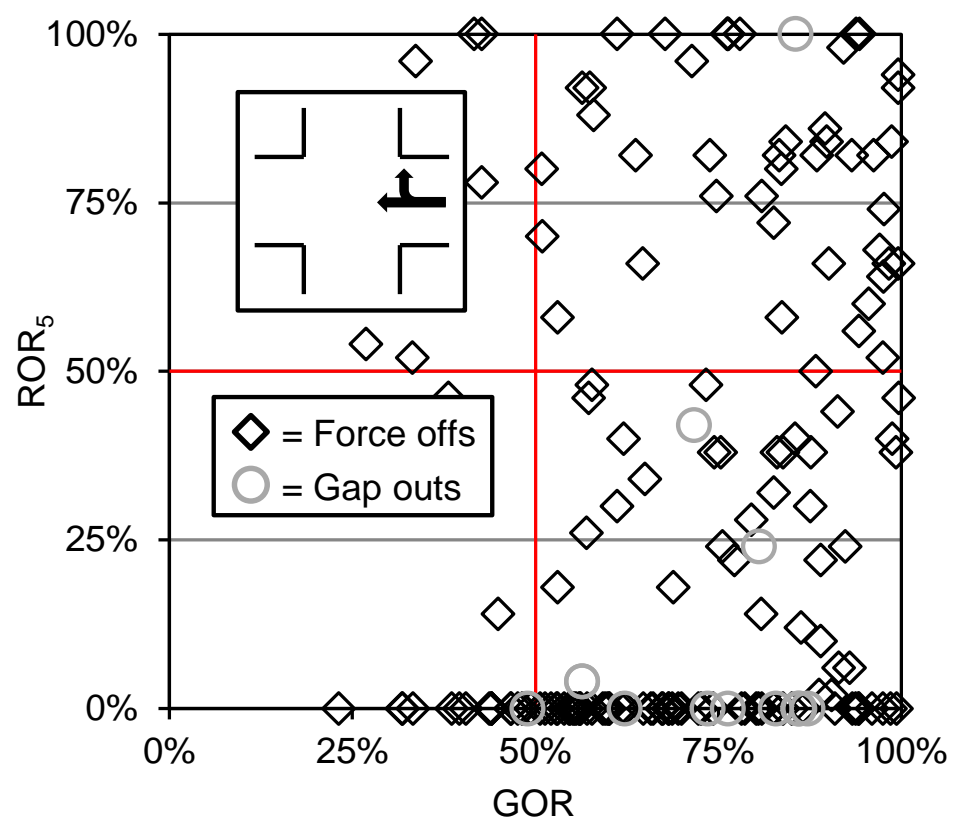

Figure 3.2.3. Phase 8 ROR/GOR plot after split adjustment. 

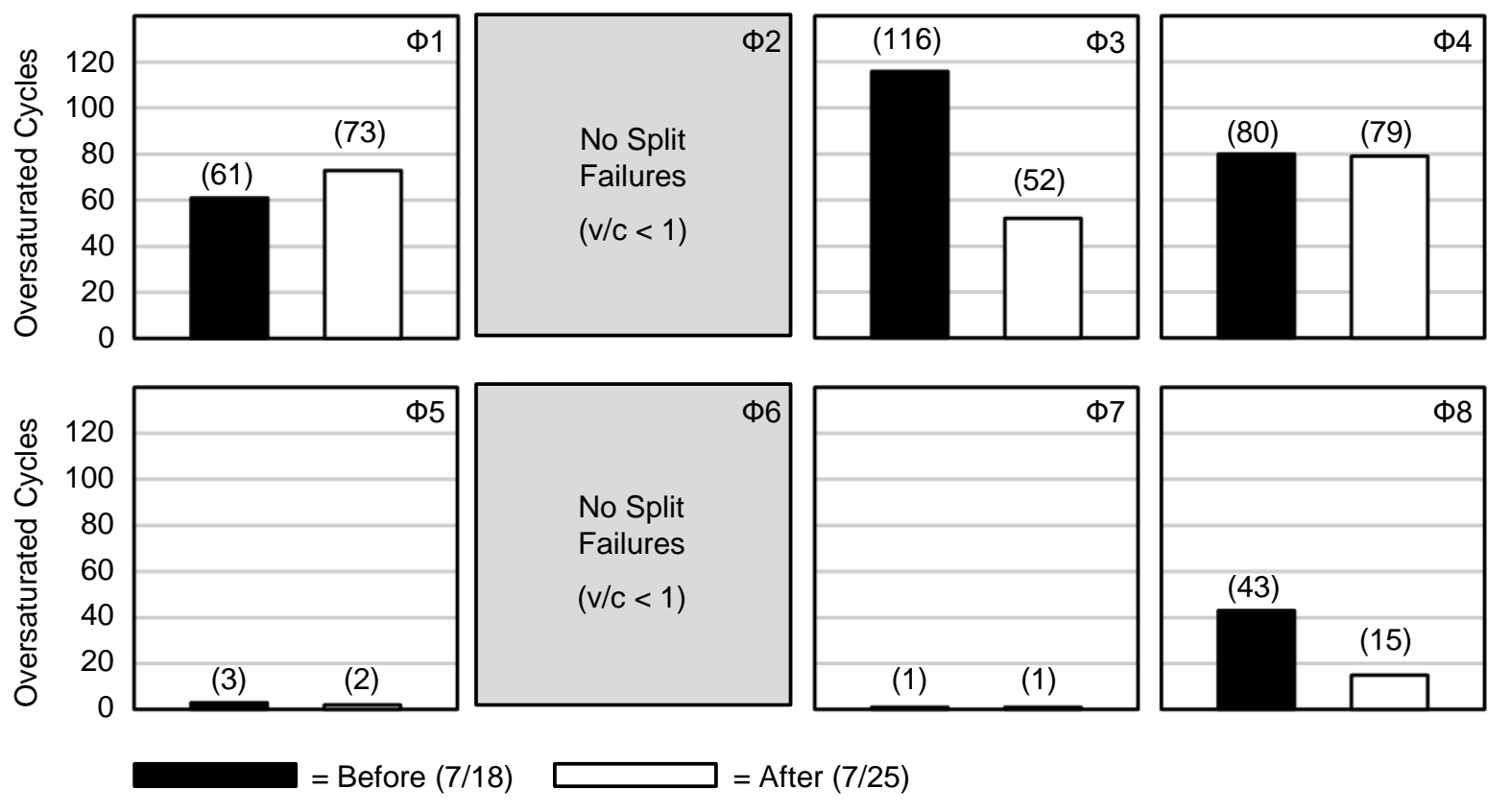

Figure 3.2.4. Before-After analysis for eight phases: Individual cycles with split failure.
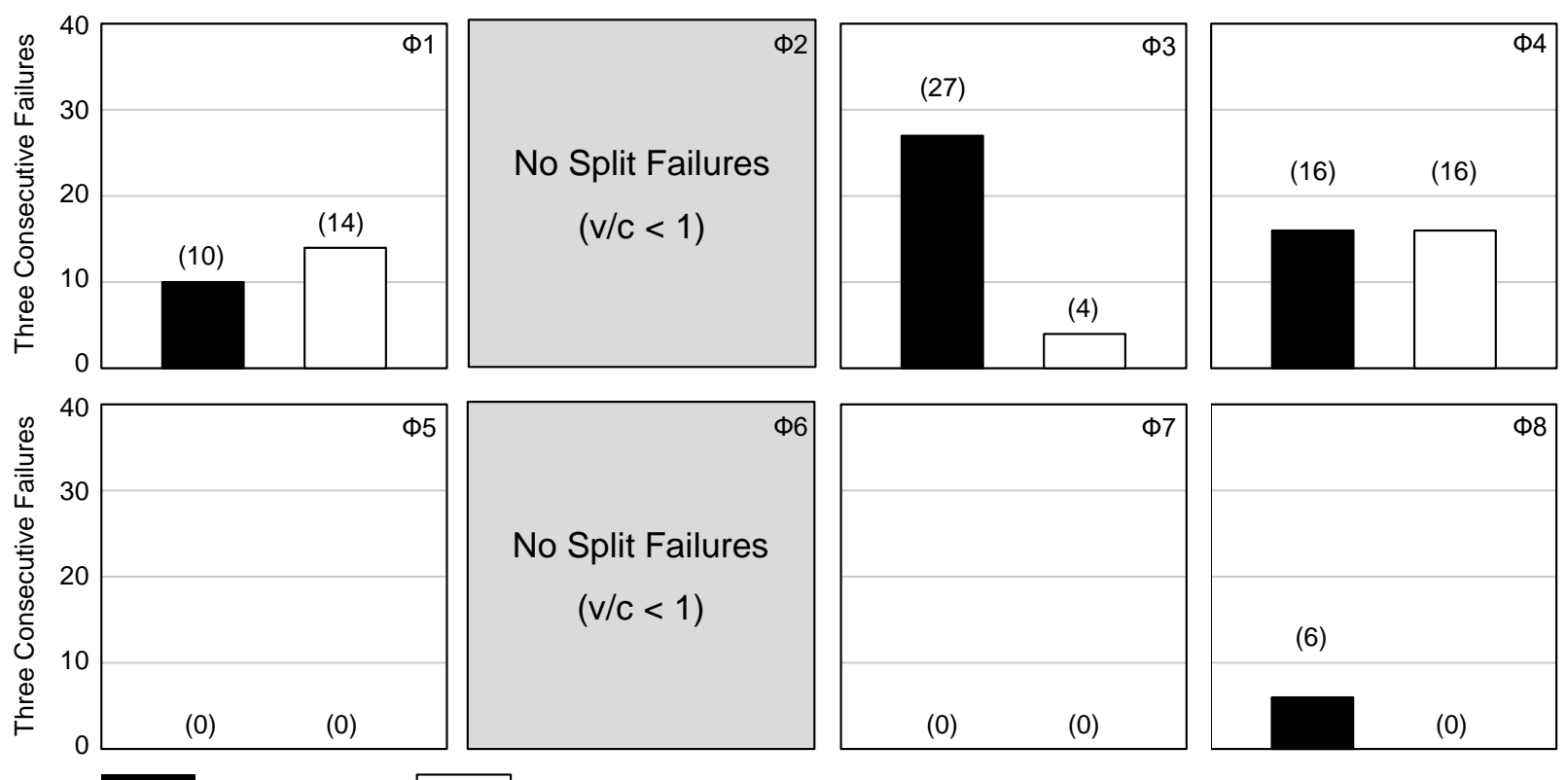

Figure 3.2.5. Before-After analysis for eight phases: Three consecutive failures. 


\subsection{Longitudinal System Analysis of Split Failures}

Performance measures can reveal operational details of individual intersections, showing how individual movements are operating at specific times of day. These views are particularly helpful when it is already known where a problem exists and when it is likely to occur. For example, performance measures can be valuable for attending to public complaint calls.

On the other hand, if the goal is to deal with issues proactively, the time and location of the relevant data views will be unknown head of time. Instead, broad coverage of the system is needed, and a method to systematically aggregate local data into system views that can pinpoint deficient operational performance.

Figure 5.3.1 shows a map of eight selected arterial corridors in the greater Indianapolis area which will be compiled for a system-wide performance comparison. These routes are all coordinated along the arterial, and most serve as commuter routes either for Indianapolis or other local communities. For the visualizations shown in this example, data was collected in October and November 2015.

In the previous section, the number of times when three split failures occurred in succession was presented as a means of measuring severe split failures. We will use this metric as the criteria for locating occurrences of split failure across this regional network.

Figure 3.3.2 shows the number of three-in-a-row split failures occurring per day on each corridor during October and November of 2015. Each bar in the chart shows the total system count for each day. Every individual color in the stack represents a subtotal for an individual corridor, as shown by the number labels at the top of the graph. The black vertical lines show when weekends occur. The weekends generally have fewer split failures, with Sundays having the lowest number. A few individual days stand out: Thanksgiving (11/26/2015) has a particularly small amount of split failures, while Black Friday (11/27/2015) has a considerably higher number. There are 153,264 occurrences of triple split failures across the eight corridor, two-month time period. In general, there appears to be a gradual decrease in this time period.

The data is sorted another way in Figure 3.3.3. Here, the total number of triple split failures is shown, while each bar represents an individual intersection within an individual corridor. Different colors in each stacked bar represent different movements at the individual intersections. Here, it is possible to very quickly assess, at a glance, which individual movements in the regional system are problematic. For example, the westbound left turn at Intersection \#1 on Corridor \#7 immediately jumps out as having the most split failures of any individual movement. The eastbound left turn at Intersection \#4 on Corridor \#6 also seems to have a high number.

These aggregated views, which have been called "split failure tickers", provide a powerful means to view system performance at a glance and to pinpoint individual problems. These are helpful not only at a top level as shown here, but one can also drill down to the individual corridor and intersection performance. 


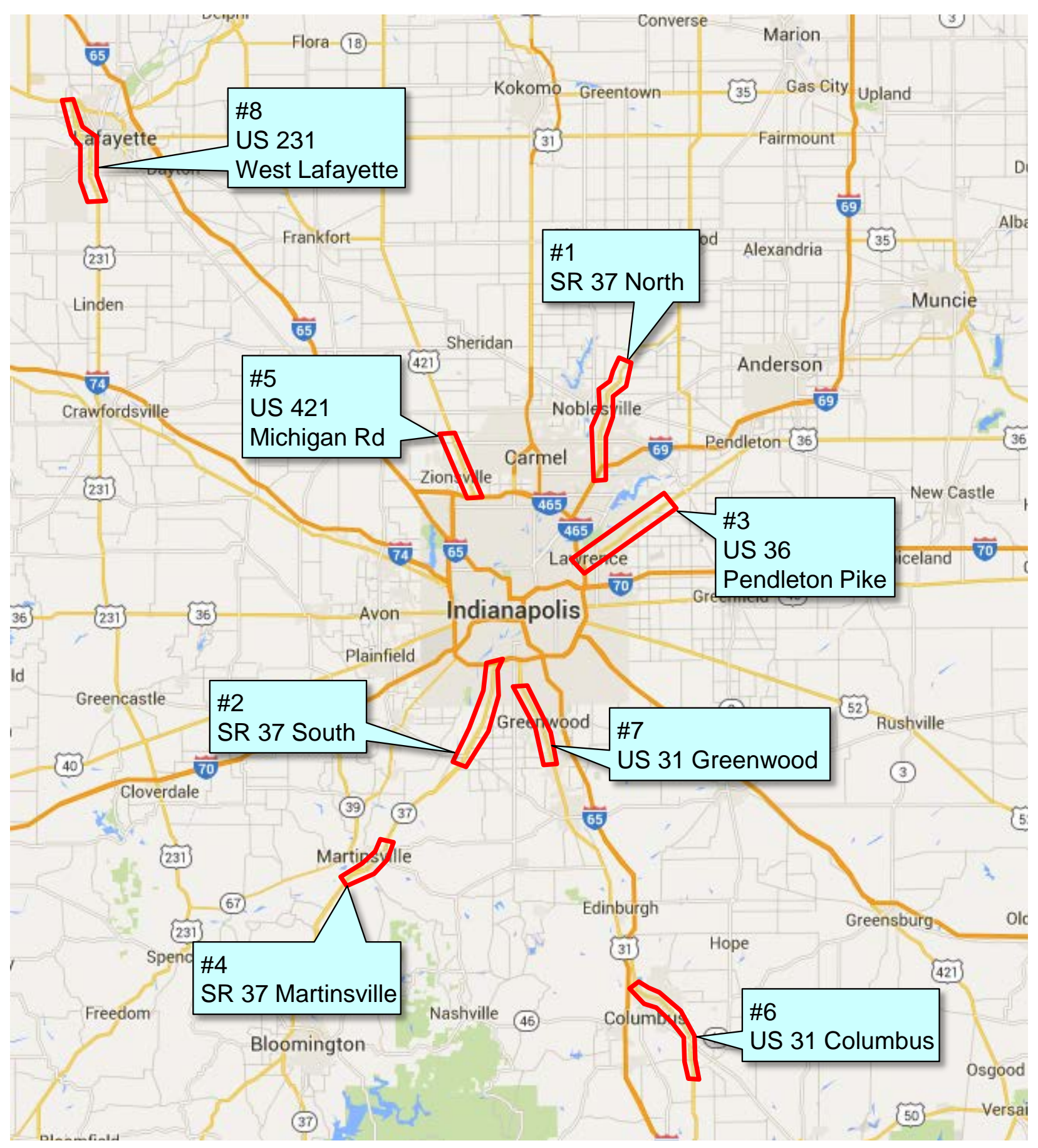

Figure 3.3.1. Map of corridors for the longitudinal analysis of Indiana arterial split failures. 


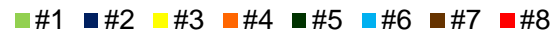

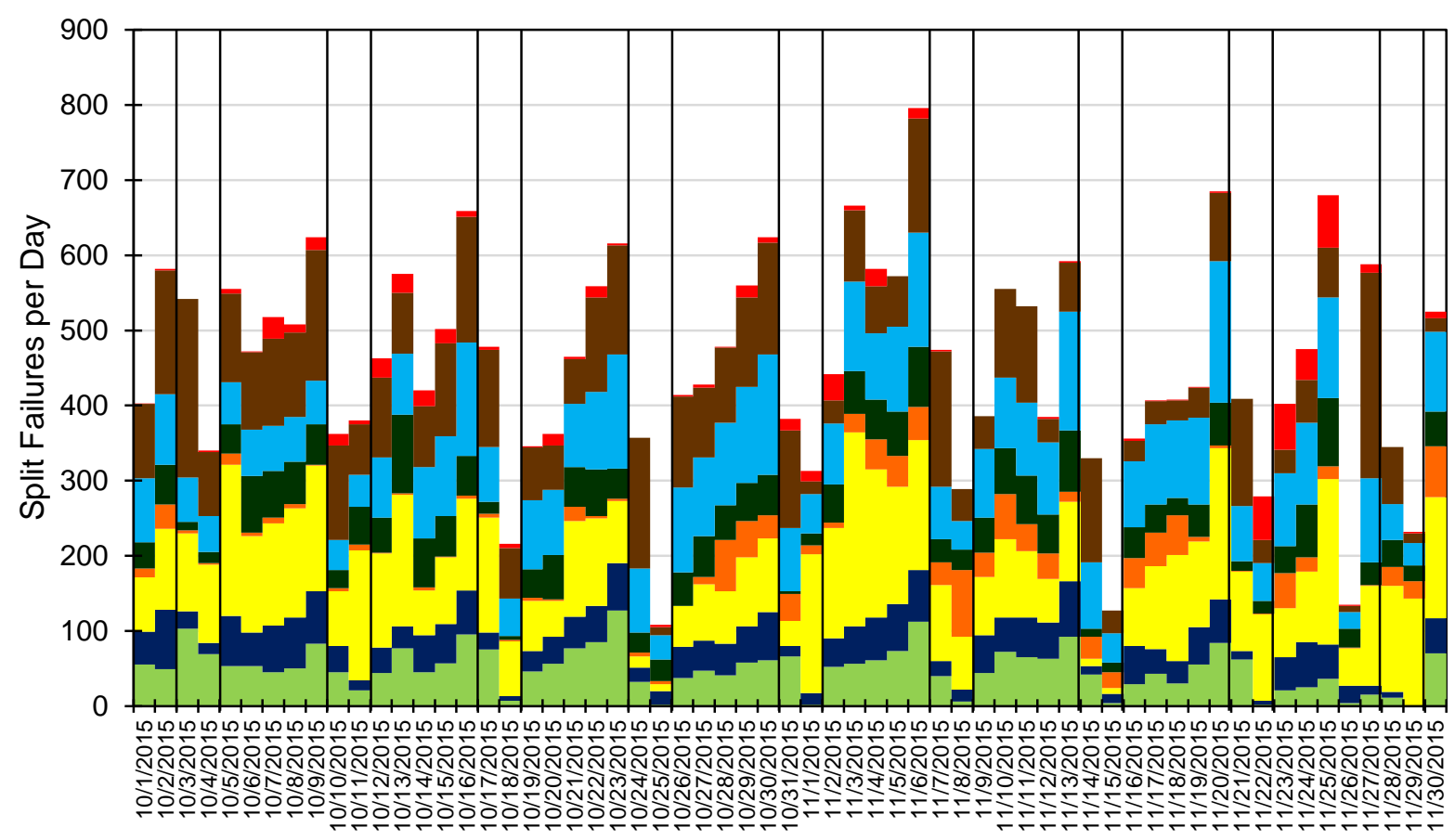

Figure 3.3.2. Number of split failures per corridor by date.

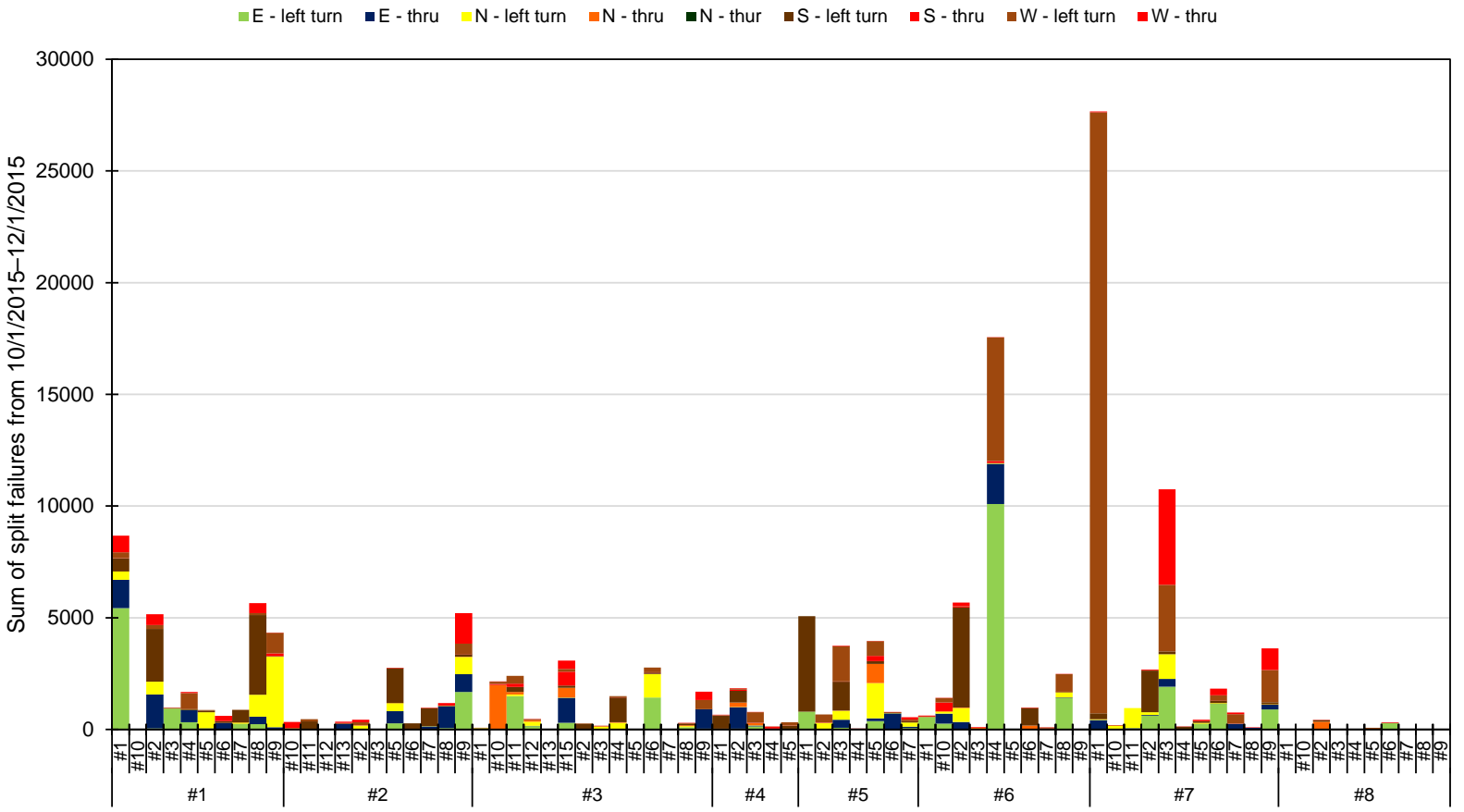

Figure 3.3.3. Total split failures per corridor and intersection. 
The system-level graphics indicated at Corridor \#7 (US 31 in Greenwood) has a couple of intersections with exceptional numbers of split failures. This gives us a reason to drill down to the individual corridor to view the performance in more detail. A map of the corridor is shown in Figure 3.3.4. There are ten intersections along this route, which serves both as a commuter corridor as well as an access route for retail and other local businesses in the Greenwood area.

A corridor split failure ticker for the US 321 Greenwood corridor is shown in Figure 3.3.5. Here, the stacked bars segment the number of split failures per intersection. The total height of the bar shows the total number of split failures in the system for each day. Intersections \#1 and \#3 stand out as having the highest number of split failures. Interestingly, the number at Intersection \#1 decreases after 11/11/2015, while Intersection \#3 has some sustained split failures throughout the entire time period. These two intersections are located at the north end of the system, which is near a large mall and other retail developments.

Figure 3.3.6 shows the same data, this time sorting by time of day rather than day of the year. Each bar represents the total number of split failures occurring per 15-minute bin throughout the 2-month analysis period. The stacked bars again show the contribution from individual intersections. For example, the split failures at Intersection \#1 are spread out from 6:00-22:00, while those at Intersection \#3 are contained within the hours 11:00-19:00. During the two-month time period, there were 2,821 occurrences of threein-a-row split failure at Intersection \#1, and 1,718 occurrences at Intersection \#3.

Finally, it is possible to drill down one level lower to the intersection level and see the contribution of individual movements. Figure 3.3.3 showed that the split failures at Intersection \#1 are mostly attributable to the westbound left turn. Therefore, we will focus on Intersection \#3, US 31 and Fry Road, to see how its individual movements are contributing to the total number.

Figure 3.3.7 shows a split failure ticker for this intersection. As before, the data is sorted by date, but now the stacked bars show the individual movements at the intersection. Three movements stand out in particular: the eastbound left, westbound left, and westbound through. These are all side-street movements, which serve traffic coming from retail destinations on both sides of the intersection. This also explains why the number of split failures is highest on Fridays and Saturdays, while the other days of the weak have relatively few; and also by Black Friday, 11/27/2015, has an extremely high number of split failures. 


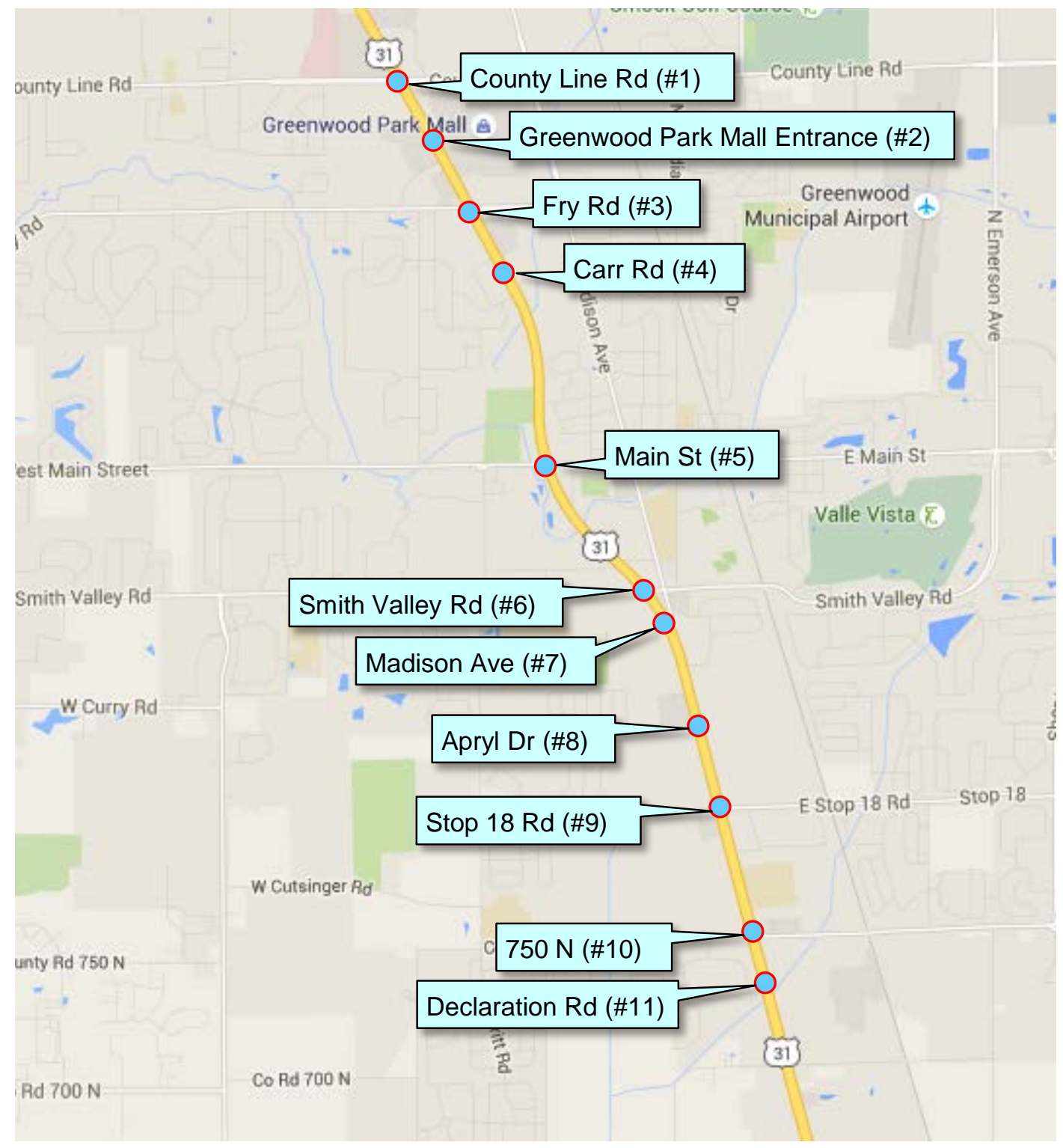

Figure 3.3.4. Map of US 31 in Greenwood, IN. 


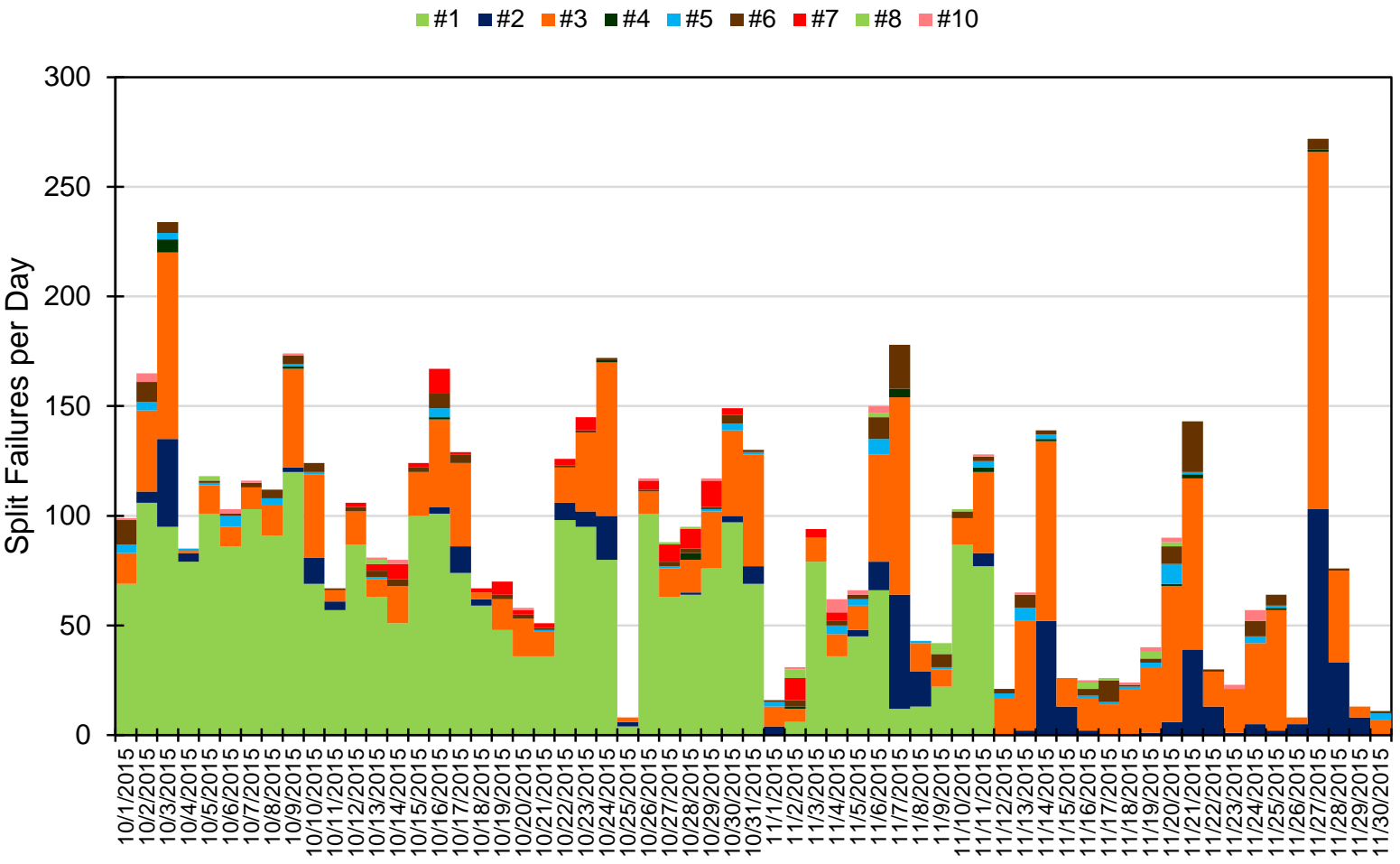

Figure 3.3.5. Split failures per intersection by date, US 31 Greenwood.

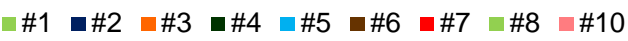

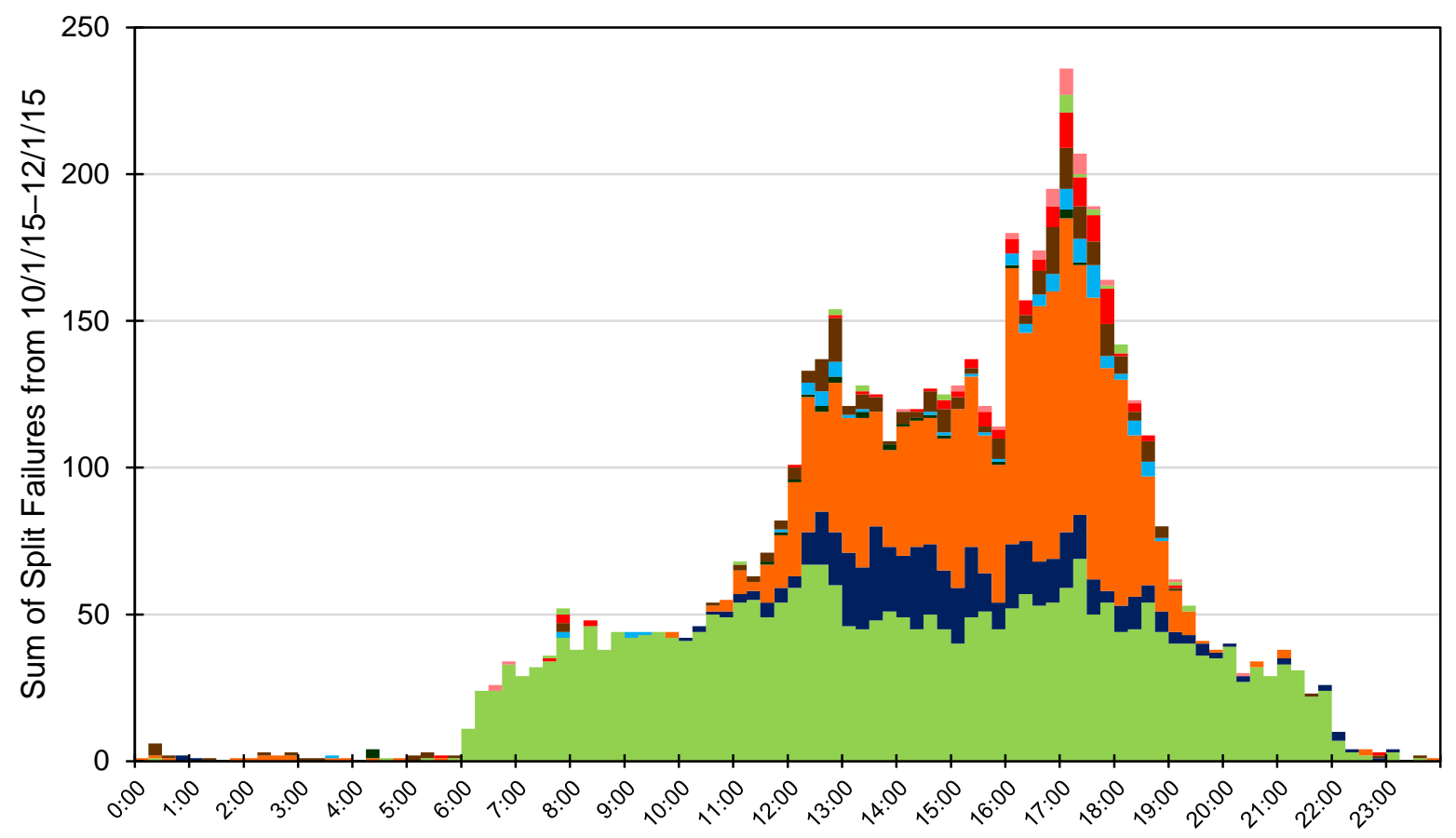

Figure 3.3.6. Split failures per intersection by time of day, US 31 Greenwood. 


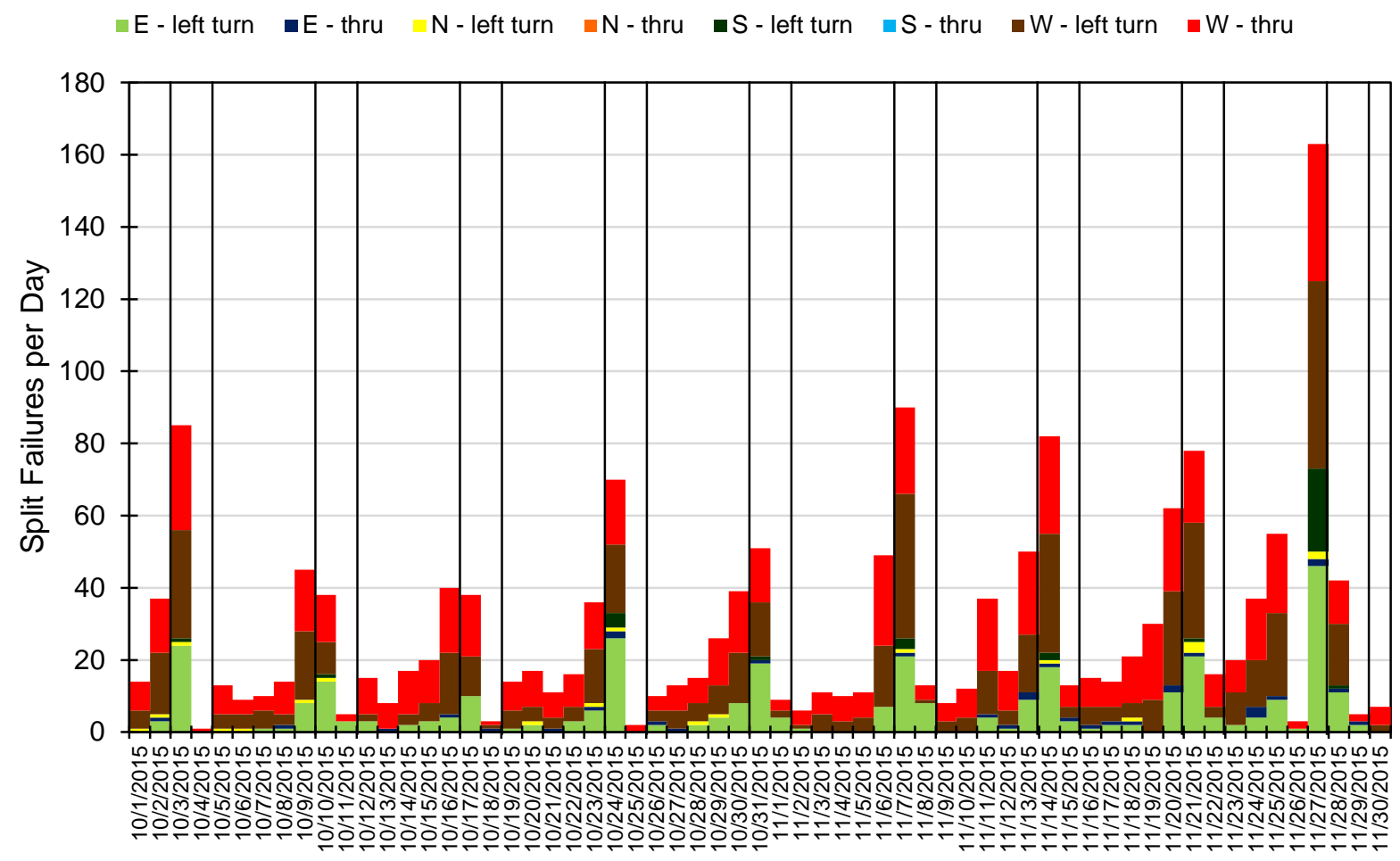

Figure 3.3.7. Split failures by movement by date, US 31 and Fry Rd. 


\section{References}

[1] C. M. Day and D. M. Bullock, "Performance Measures of Interrupted-Flow Roadways using ReIdentification and Signal Controller Data," FHWA SBIR 3, 2015.

[2] S. E. Young, "BluSTATs Processing Manual," Traffax Inc.

[3] S.E. Young, M. Hamedi, E Sharifi, R.M. Juster, K. Kaushik, and S. Eshragh, "I-95 Corridor Coalition Vehicle Probe Project Validation of Arterial Probe Data", July 2015. 


\section{Report Sponsor}

The "Small Business Innovation Development Act of 1982" (Pub. L. No. 97-219), along with reauthorizing legislation (Pub. L. No. 99-443 and Pub. L. No. 102-564, the "Small Business Research and Development Enhancement Act of 1992"), seeks to encourage the initiative of the private sector and to use small business effectively to meet federal research and development objectives. To comply with statutory obligations of the Act, the U.S. Department of Transportation established the Small Business Innovation Research (SBIR) Program, which conforms to the guidelines and regulations provided by the Small Business Administration. Annually, small businesses are solicited to submit innovative research proposals that address the high-priority requirements of the U.S. Department of Transportation and that have potential for commercialization.

This report was developed through a partnership between Traffax, Inc., and Purdue University with funding from a Phase III SBIR contract (DTFH6114C00035) with the Federal Highway Administration. The project, entitled "Sensor Fusion and MOE Development for Off-Line Traffic Analysis of Real Time Data," created and refined methods and tools for the characterization of performance along arterial corridors.

\section{Publication}

This report is part of a series of reports published in collaboration with USDOT, Traffax, Inc., and Purdue University. The full report series is available for download at http://docs.lib.purdue .edu/apmtp/.

\section{Open Access and Collaboration with Purdue University}

The Indiana legislature established the Joint Highway Research Project in 1937. In 1997, this collaborative venture between the Indiana Department of Transportation and Purdue University was renamed as the Joint Transportation Research Program (JTRP) to reflect state and national efforts to integrate the management and operation of various transportation modes. Since 1937, the JTRP program has published over 1,600 technical reports. In 2010, the JTRP partnered with the Purdue University Libraries to incorporate these technical reports in the University's open access digital repository and to develop production processes for rapidly disseminating new research reports via this repository. Affiliated publications have also recently been added to the collection. As of 2017, the JTRP collection had over 1.5 million downloads, with some particularly popular reports having over 20,000 downloads. 